DOE/NASA/0341-1

NASA CR-174991

AV-FR-85/802

\title{
Development and Testing of Tip Devices for Horizontal Axis Wind Turbines
}

G.W. Gyatt and P.B.S. Lissaman AeroVironment Inc.

May 1985

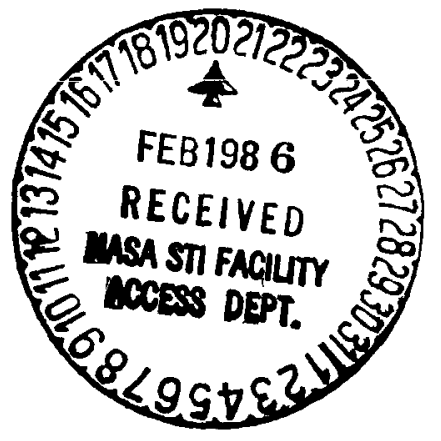

(NASA-CB-174991) DEVEIOPHENT AND TESTING OF

N86- 18774 IIP DEVICES FOR HCRIZCNTAL AXIS RIND

TURBINES Final Report (AeroVironment, Inc.)

$86 \mathrm{P}$ HC A05/AF 01 CSCL 10A

Dnclas

$63 / 44 \quad 05428$

Prepared for

NATIONAL AERONAUTICS AND SPACE ADMINISTRATION

Lewis Research Center

Under Contract DEN 3-341

for

U.S. DEPARTMENT OF ENERGY

Conservation and Renewable Energy

Wind Energy Technology Division 
DOE/NASA/0341-1

NASA CR-174991

AV-FR-85/802

\section{Development and Testing of Tip Devices for Horizontal Axis Wind Turbines}

G.W. Gyatt and P.B.S. Lissaman AeroVironment Inc.

Monrovia, California 91016-3424

May 1985

Prepared for

National Aeronautics and Space Administration

Lewis Research Center

Cleveland, Ohio 44135

Under Contract DEN 3-341

for

U.S. DEPARTMENT OF ENERGY

Conservation and Renewable Energy

Wind Energy Technology Division

Washington, D.C. 20545

Under Interagency Agreement DE-A101-76ET20320 
DEFINITIONS OF SYMBOLS

INTRODUCTION

ANALYSIS METHOD

DESIGN

FABRICATION

RESULTS

DISCUSSION OF RESULTS

CONCLUSIONS AND RECOMMENDATIONS 61

REFERENCES

APPENDICES

A Specification Summary of Test Turbine

B Catalog of Tip Device Designs and Their Predicted Performance 


\section{DEFINITIONS OF SYMBOLS}

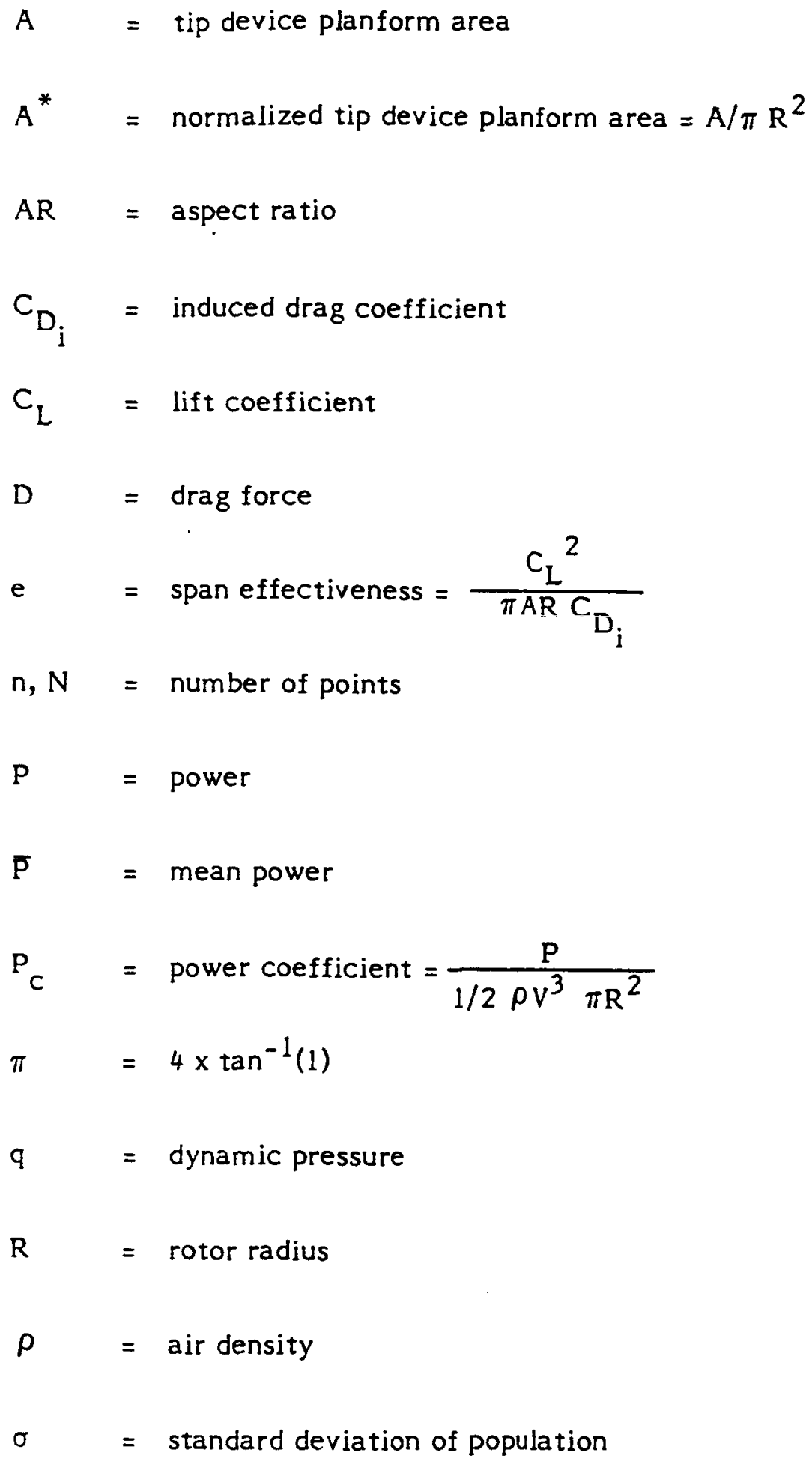




\section{DEFINITION OF SYMBOLS (Continued)}

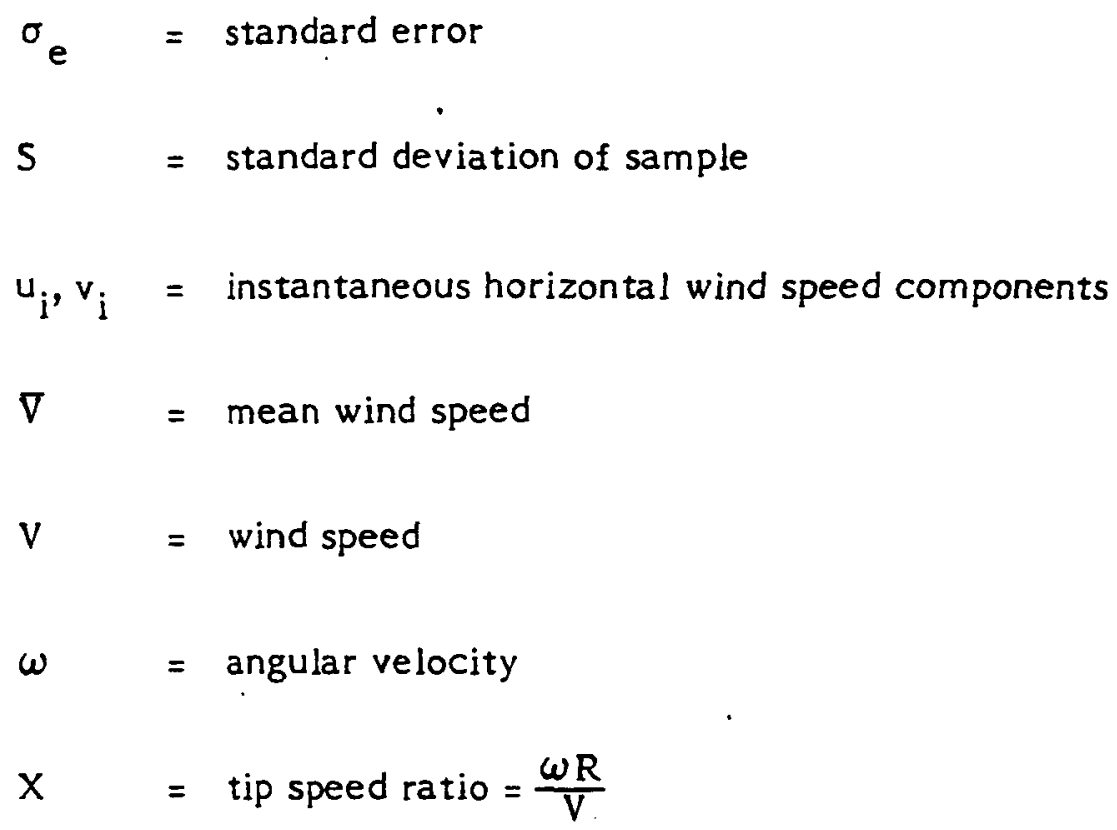




\section{INTRODUCTION}

This program was carried out to design and test advanced tip shapes for horizontal axis wind turbine rotor blades in order to reduce tip losses and improve performance with a minimal cost penalty.

A typical modern horizontal axis wind turbine can suffer as much as a $10 \%$ power reduction due to tip losses. Tip losses are caused by vortex shedding near the tip of each blade. This vortex increases the axial and circumferential interference factors experienced by the tip region of the blade. The result is that the outboard portion of the blade operates less effectively than the inboard sections which are farther from the main region of influence of the vortex.

One well-known method for reducing tip losses on rotors is to increase the number of blades. This has the effect of spreading out the shed vorticity over the wake boundary in a more uniform manner and, as a result, the interference on each blade is reduced and the rotor more closely resembles an ideal actuator disk. This method of increasing the number of blades is not, however, very cost effective. Therefore, a method is sought for reducing tip losses which does not add significant structure, complexity or weight to the wind turbine. Tip devices have the potential for reducing tip losses without these penalties.

Some tip devices have already been investigated for use on wind turbines. They fall into two general categories: (1) relatively large tip vanes with a span up to one-half the rotor radius, which are designed to increase the power output by 100 to 200\% through flow augmentation referred to as a dynamic inducer vane (Gyatt et al., 1982), and (2) smaller tip plates with a span roughly equal to the chord of the rotor tip, which are designed to pop out as spoilers for overspeed control. However, neither of these devices are dedicated to tailoring the flow at the blade tips in order to reduce tip losses.

A considerable amount of work has already been done on the design of advanced tip shapes for wings to reduce tip losses. These include winglets and tip sails and are reported in the list of references at the end of this report. 
sails and are reported in the list of references at the end of this report. Significantly, however, there has been no research to adapt these small aerodynamic lifting surfaces to wind turbines. A brief summary of the highlights of previous work on tip devices is given below.

Winglets are small, planar lifting surfaces mounted at the tips of a wing in a plane approximately perpendicular to the wing plane with the objective of reducing the drag coefficient of the system more than could be achieved by a simple wing-tip extension with the same structural weight penalty. A sketch of a winglet developed for a jet transport is shown in Figure 1 (Flechner et al., 1976). The span of the main winglet is roughly equal to the chord of the wing tip, while its chord is about one-half the chord of the wing tip. Wind tunnel measurements on winglets showed a $20 \%$ reduction in induced drag with a projected $7 \%$ saving in fuel consumption for the jet transport (Whitcomb, 1976).

Figure 2 shows a vortex diffusing vane designed for the "Thrush" agricultural aircraft taken from Hackett (1981).

Smaller multi-element winglets, so called wing tip sails, have also been developed for aircraft. A sketch of a wing tip sail is shown in Figure 3 where its similarity to the alula feathers at the tip of a bird's wing can be seen. The span and chord of the wing tip sail is only about one-third and one-fifth the chord of a wing tip, respectively. There are three sails on each wing tip. Wind tunnel measurements and flight tests with three sails per side showed a 28 to $29 \%$ reduction in induced drag (Spillman, 1978).

Table 1 summarizes tests of tip devices on aspect ratio 3 wings as reported by Hackett and Phillips (1980). The span effectiveness e is defined by

$$
c_{D i}=\frac{c_{L}{ }^{2}}{e \pi A R}
$$

where $C_{D i}$ and $C_{L}$ are the induced drag and lift coefficient, based on original wing area, and $A R$ is the aspect ratio. 

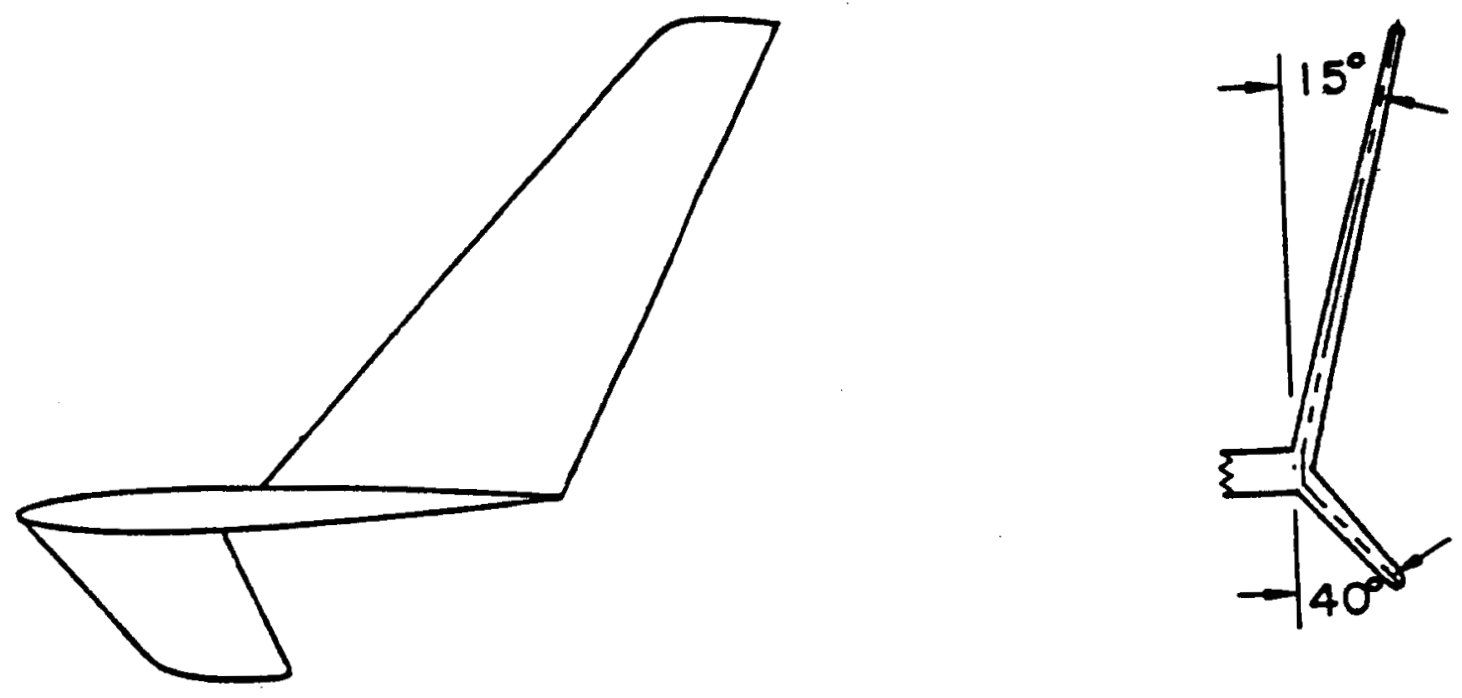

FIGURE i. Wingiets developed for a jet transport.

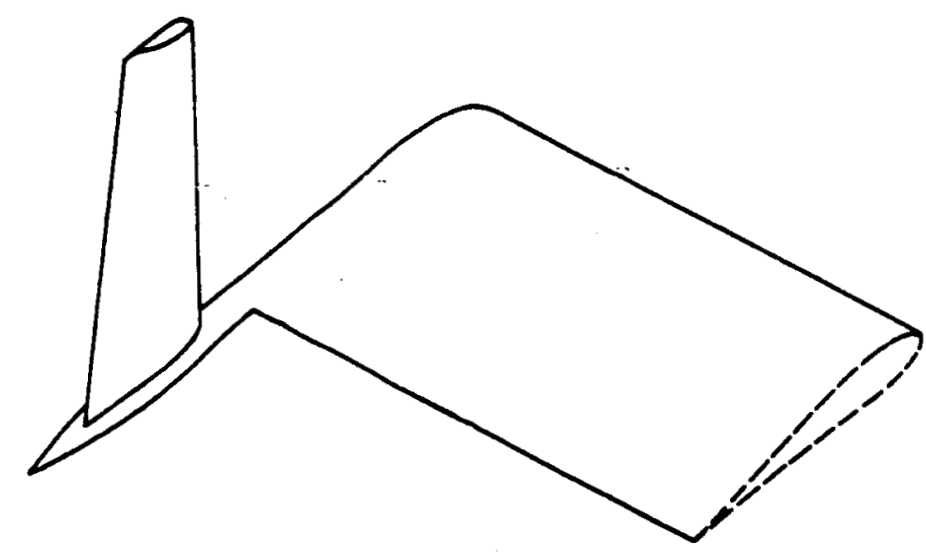

FIGURE 2. Aft wing tip vane for "Thrush" aircraft. 

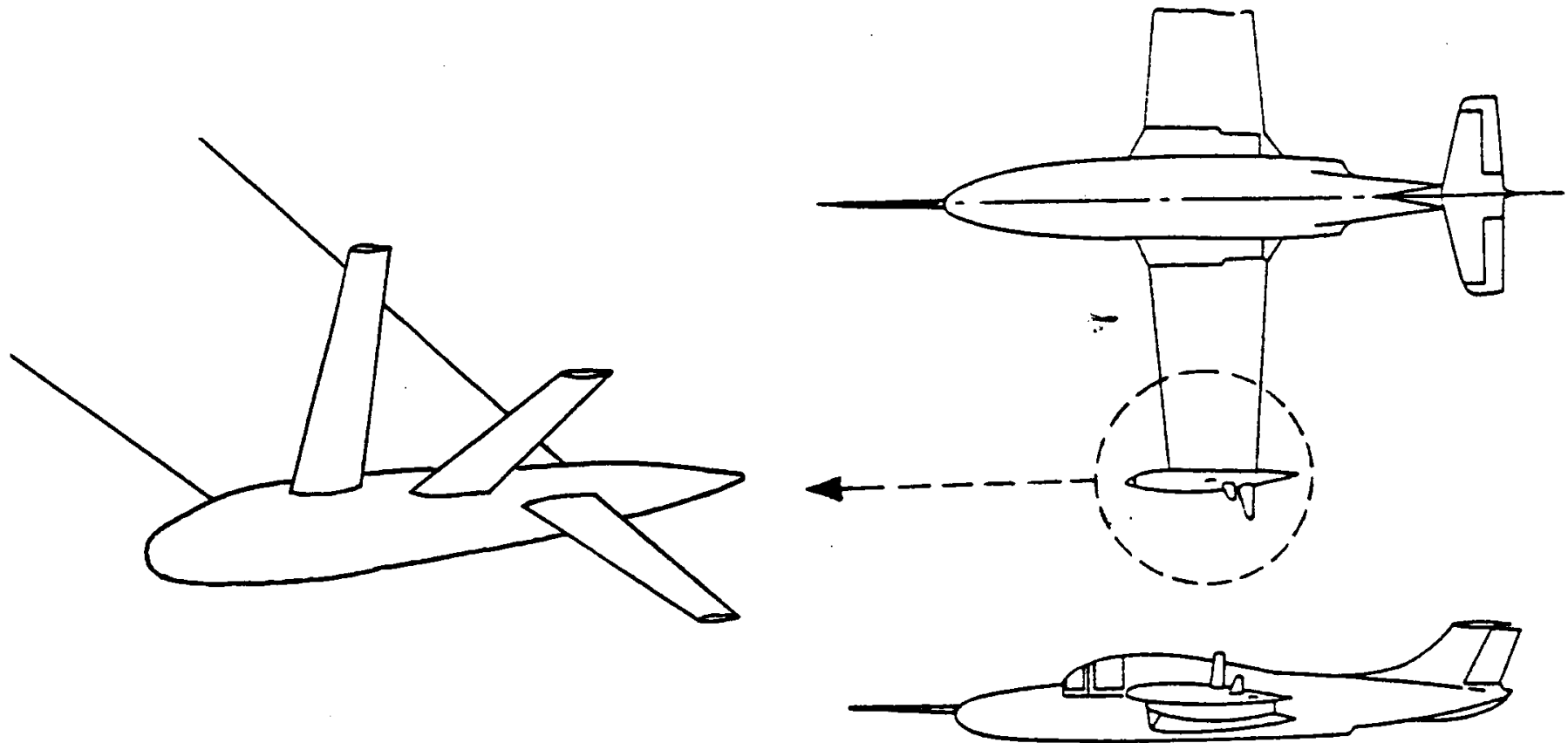

FIGURE 3. Wing tip sails tested on Paris aircraft.

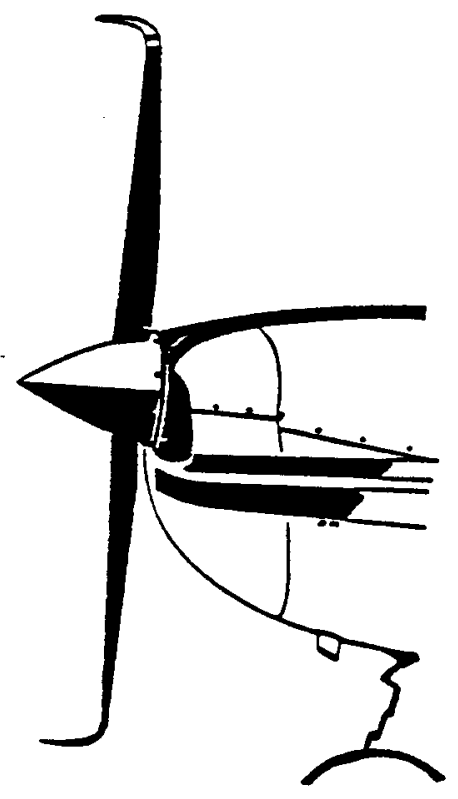

FIGURE 4. General aviation propeller with proplets. 
TABLE 1. Effect of tip devices on an aspect ratio three rectangular planform wing.

\begin{tabular}{|l|c|c|}
\hline \multicolumn{1}{|c|}{ Tip Device } & $\begin{array}{c}\text { Extra } \\
\text { Area (\%) }\end{array}$ & $\begin{array}{c}\text { Span } \\
\text { Effectiveness }\end{array}$ \\
\hline \hline None & 0 & 0.84 \\
Simple Extension & 15 & 1.14 \\
Single Winglet & 15 & 1.24 \\
Wing Tip Sails & 9 & 1.38 \\
Vortex Diffuser Vane & 15 & 1.20 \\
\hline
\end{tabular}

Source: Hackett and Phllips (1980). 
Nonplanar lifting surfaces have already been adapted to propellers and are referred to as proplets. A sketch of a proplet on a general aviation propeller is shown in Figure 4. Theoretical calculations show that proplets can increase the propulsive efficiency of a fixed diameter propeller by one to five percent (Sullivan et al., 1982).

The results of this work on wings and propellers suggest that a properly designed tip shape for a wind turbine could reduce tip losses significantly and lead to a more cost-effective rotor design than presently available. The program described involves the design, fabrication, and testing of tip devices on a commercially available wind turbine, the Carter Wind Systems Model 25, specifications for which may be found in Appendix A.

\section{ANALYSIS METHOD}

The first stage of this task was to select a computer code for modeling the effects of tip devices on wind turbine rotors. Of the two available codes, one had been developed by Drs. Li Ko Chang and John P. Sullivan of Purdue University to study propeller performance and the other by Dr. Ilan Kroo of NASA Ames which had been designed primarily for the investigation of aircraft configurations.

Both are written in FORTRAN and based on vortex line theory, although with Kroo's code it is possible to simulate a vortex lattice by arranging a series of lifting lines one behind the other; this could be done, for example, to model a low aspect ratio wing using several high aspect ratio elements with the trailing edge of one touching the leading edge of the next. Both codes presently constrain the path of each shed vortex to a cylindrical helix without radial expansion or variation in axial flow; i.e., neither code includes the effect of the induced velocity field of the vortices on themselves. The most significant difference between the two codes is in their abilities to model tip devices. The available form of the Chang/Sullivan code models any tip device by a single vortex line extending from the blade tip, and therefore cannot include the effects of such details as taper or twist; Kroo's code, however, is more flexible in this regard and can represent a tip device in as much, or more, detail as the main blade using, within reason, as many horseshoe vortices 
as required. The Chang/Sullivan code was written over a number of years by several contributors and is still undergoing development; it was decided not to attempt to modify the code because of the amount of work involved. Kroo's code was therefore selected for the analysis task. Auxiliary input and output codes were written to simplify and speed up use of Kroo's code and to ensure better documentation of resuits.

Kroo arranged for AV to run his code on the "FAR" VAX-750 computer at NASA-Ames. Kroo's code is a general, nonplanar, discrete Weissinger code; the Weissinger method imposes the boundary condition of tangential flow at the $3 / 4$ chord point of any lifting area element. It is capable of computing forces, moments and velocity distributions of multi-element, nonplanar lifting surfaces.

Kroo's code requires linear taper and twist of wing elements and assumes a lift curve slope of $2 \pi$ per radian. Viscous drag effects are calculated from a "look-up" table of profile drag coefficients stored in a subroutine. The chord and pitch distributions of the Carter blade are shown in Figures 5 and 6. Although neither of these parameters vary linearly with radius, as required by Kroo's code, it can be seen that both are almost constant for the normalized radius greater than 0.5 which constitutes the outer $75 \%$ of the swept area. Since the analysis task is concerned only with detecting differences in performance with and without tip devices, and not absolute values, and the tip devices themselves are expected to influence only the flow fields near the tips, it was felt that the Carter blade could be adequately modeled as an untwisted, untapered section of normalized chord $0.065\left(12.5^{\prime \prime}\right)$ at a pitch setting of $-1^{\circ}$ for the purpose of using Kroo's code.

The tip airfoil section of the Carter blade is the NACA 23012 airfoil, while the root uses the thicker but similar NACA 23021. The tip section operates at a Reynolds number of approximately $1.4 \times 10^{6}$. The performance of the 23012 in these conditions as reported by different test facilities is shown in Figures 7 and 8. The lift curve approximates closely to the value $C_{L}=0.11$ per degree used by Kroo. Again, because only differences in performance are important, it is felt that Kroo's approximation is adequate for the whole Carter blade provided that proper care is taken in interpreting results which include sections operating in the stalled region beyond $15^{\circ}$ angle of attack. 


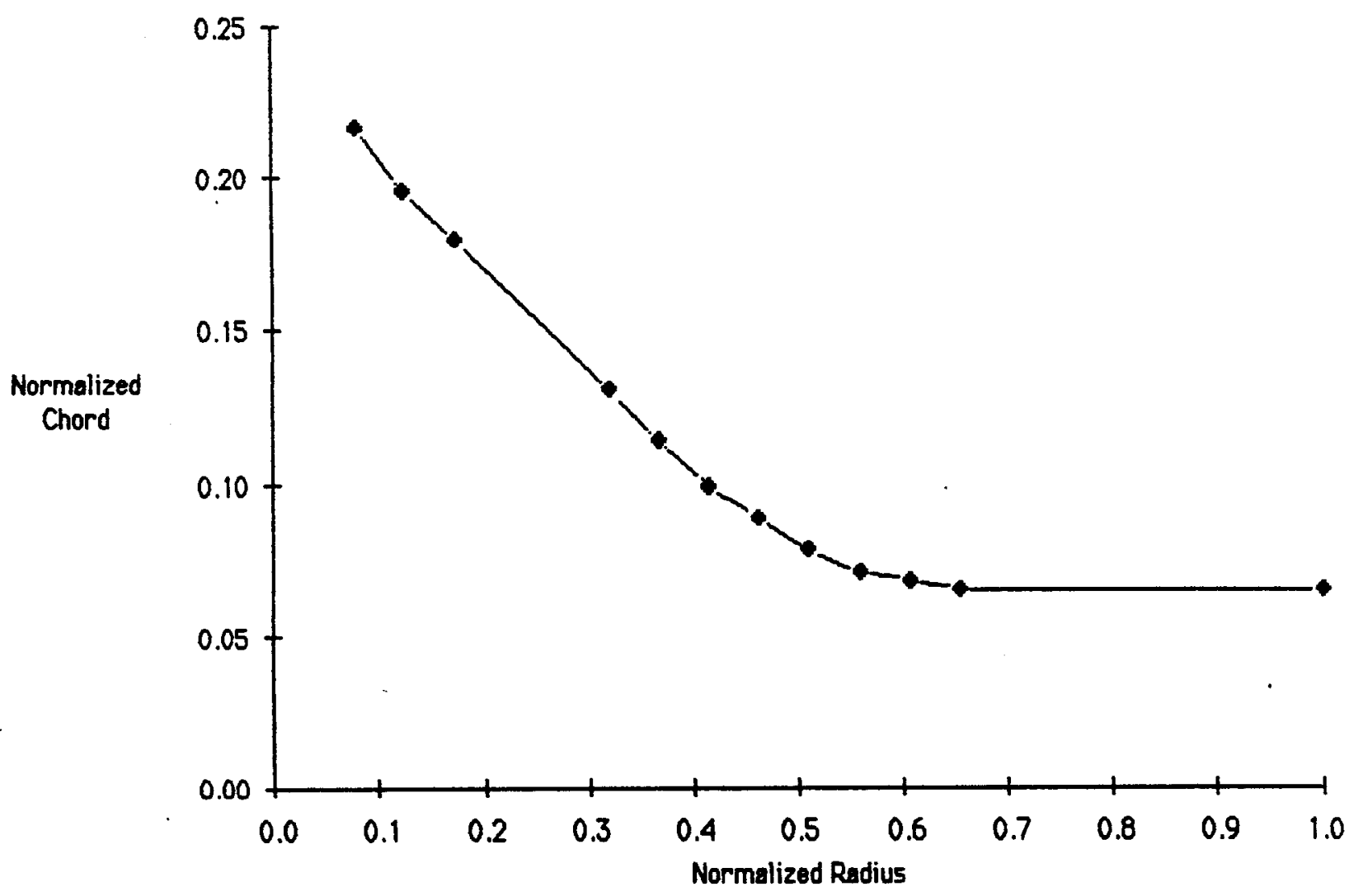

FIGURE 5. Carter blade chord distribution. 


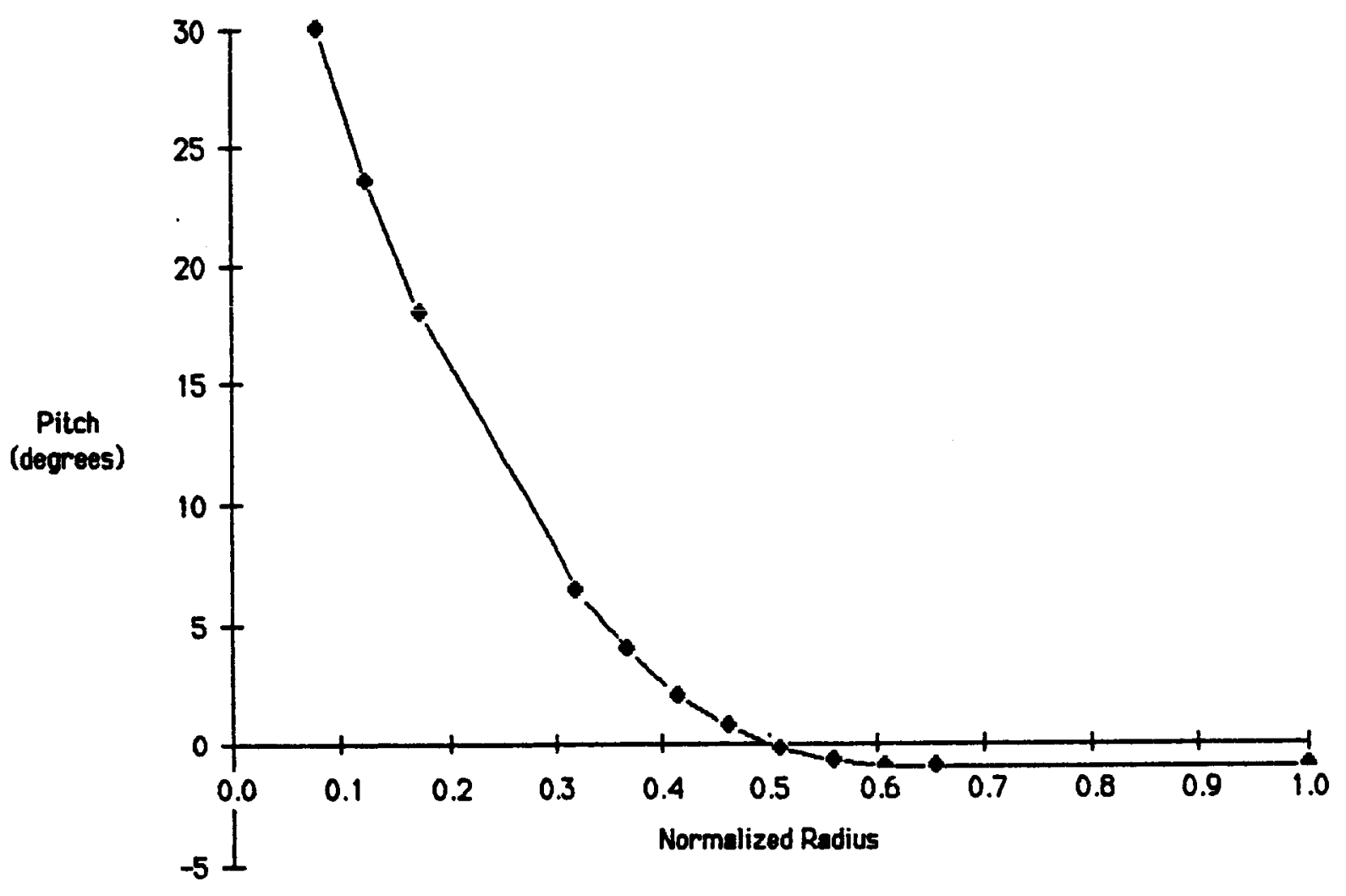

FIGURE 6. Carter blade pitch distribution. 


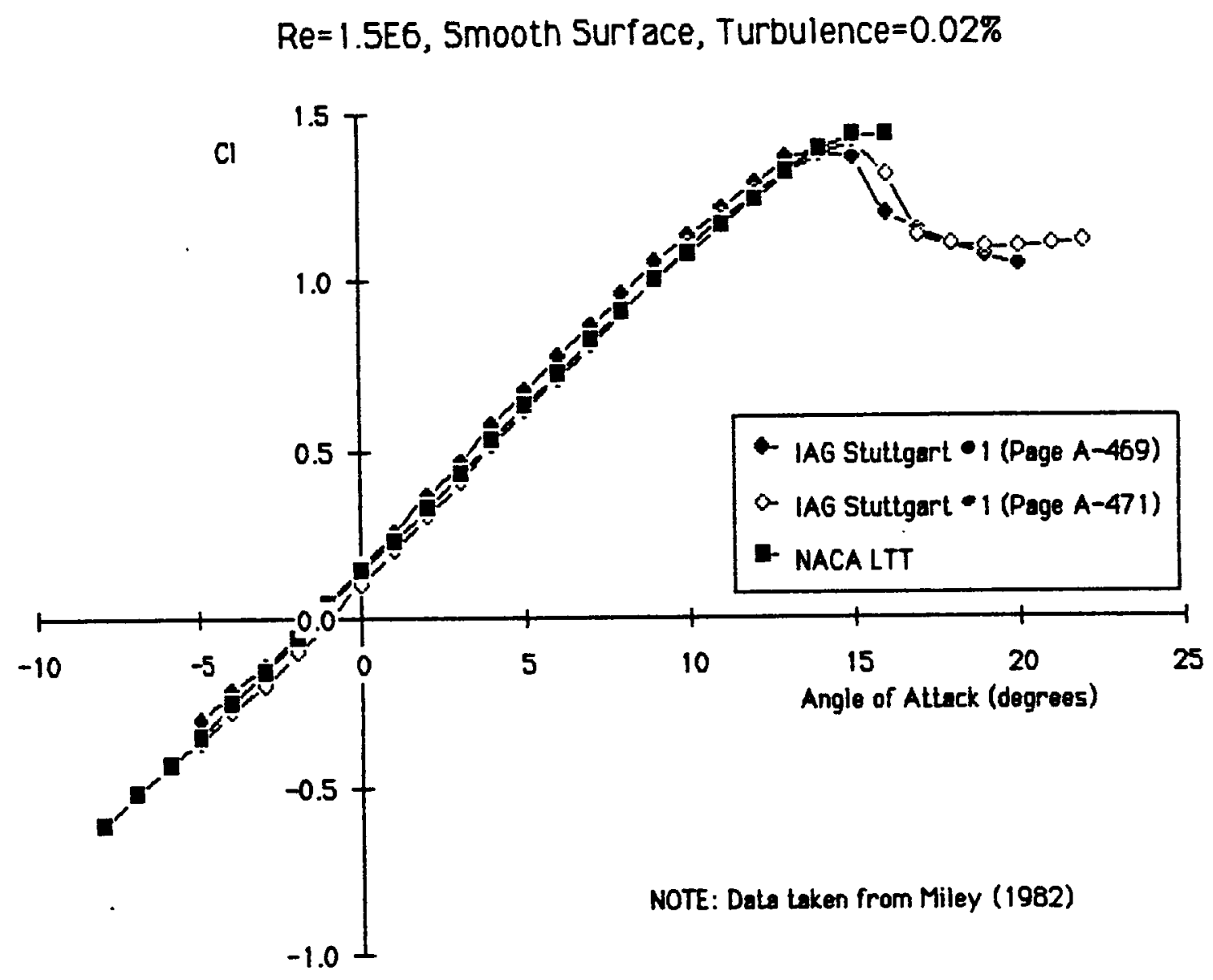

FIGURE 7. Lift of NACA 23012 as reported by different facilities. 


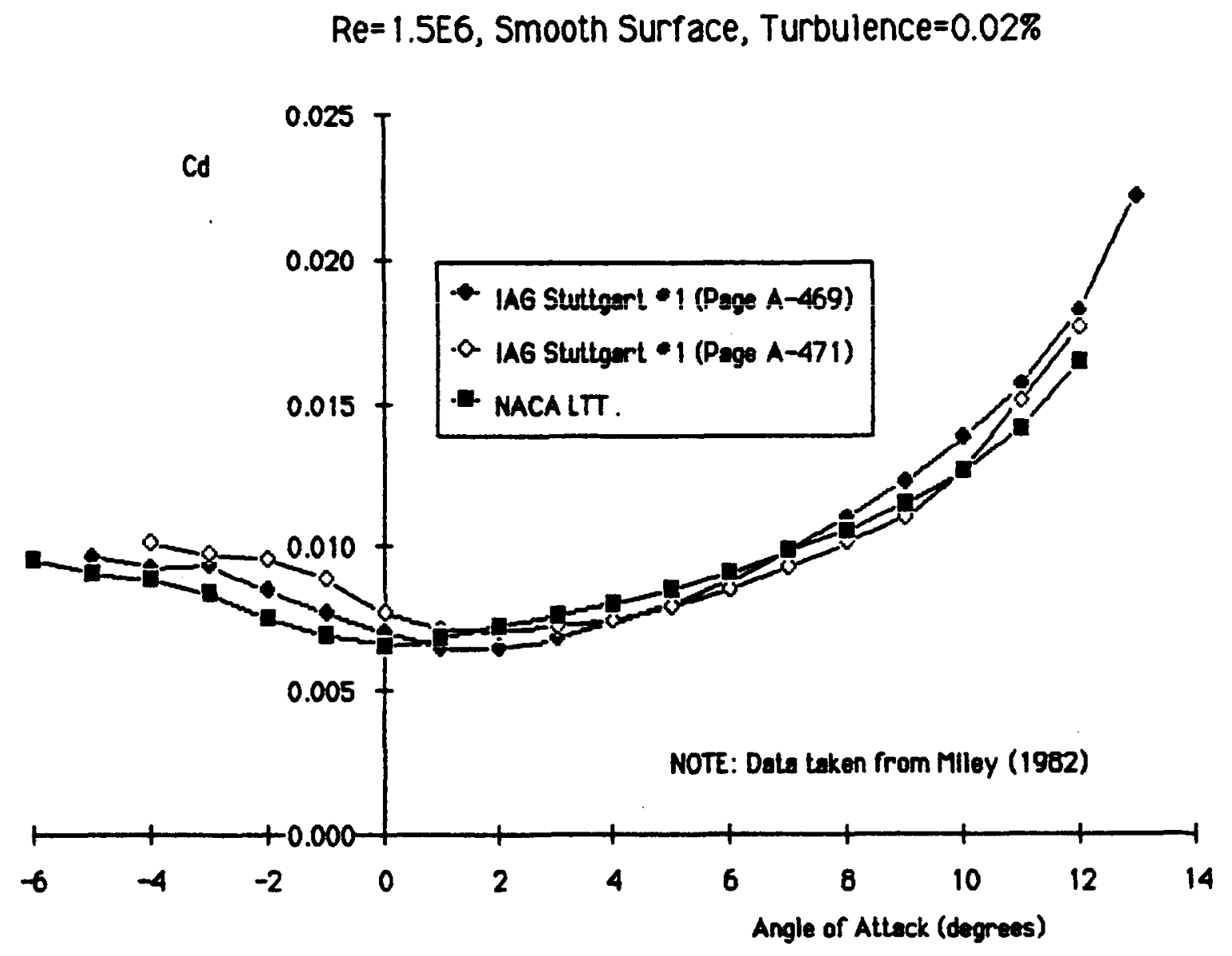

FIGURE 8. Drag of NACA 23012 as reported by different facilities. 
Before the design phase began the results of Kroo's vortex line model called LINAIR were compared with those of AV's PROP code.

The PROP code, developed in 1976 by Wilson and Lissaman (1974) under a grant from the National Science Foundation, is based on Glauert momentum strip theory, in which each annulus behaves independently and is unaffected by its neighbors; Figures 9 and 10 show a comparison of the models when exercised for a simple constant chord $(0.05 \mathrm{R})$, untwisted rotor at two pitch settings. For added simplicity the drag coefficient of the blades was taken as zero (i.e., inviscid flow) which explains the high $C_{p}$ max of 0.54 predicted by the PROP code.

While good agreement was observed for a lightly loaded rotor (corresponding to $+3^{\circ}$ blade setting), Kroo's predictions for thrust and especially power coefficient were too large at high tip speed ratios for a heavily loaded rotor. Kroo's model gives values which increase almost linearly with tip speed ratio, and indicate power coefficients greater than the Betz limit, while the PROP code values start to roll off at tip speed ratios around eight, as expected. Even though Kroo's code does not handle the stalled region correctly, the values for $P_{c}$ should not exceed Bet 2 limit. In the past, the PROP code has been compared with experimental data for both propellers and wind turbines as well as with other analytical models, and has shown good agreement. After examining the code and discussing the results with Dr. Kroo, it was thought that the differences may be due to one or a combination of the following reasons:

1) Errors either in the fluid mehanical formulation or in the computer code.

2) Inadequate geometric modeling of the helical vortex. Each shed vortex is represented not by a continuous, smooth infinitely long helix, but by a finite number of straight vortex lines connected end to end in the approximate shape of a helix. The number of these straight line segments in each shed vortex is fixed at 50 and each vortex is carried 2.4 rotor diameters downstream; at high tip speed ratios, this means that the angle subtended by each leg on the axis of rotation is quite large (e.g., $70^{\circ}$ at $X=12$ ) and inaccuracies will result. 


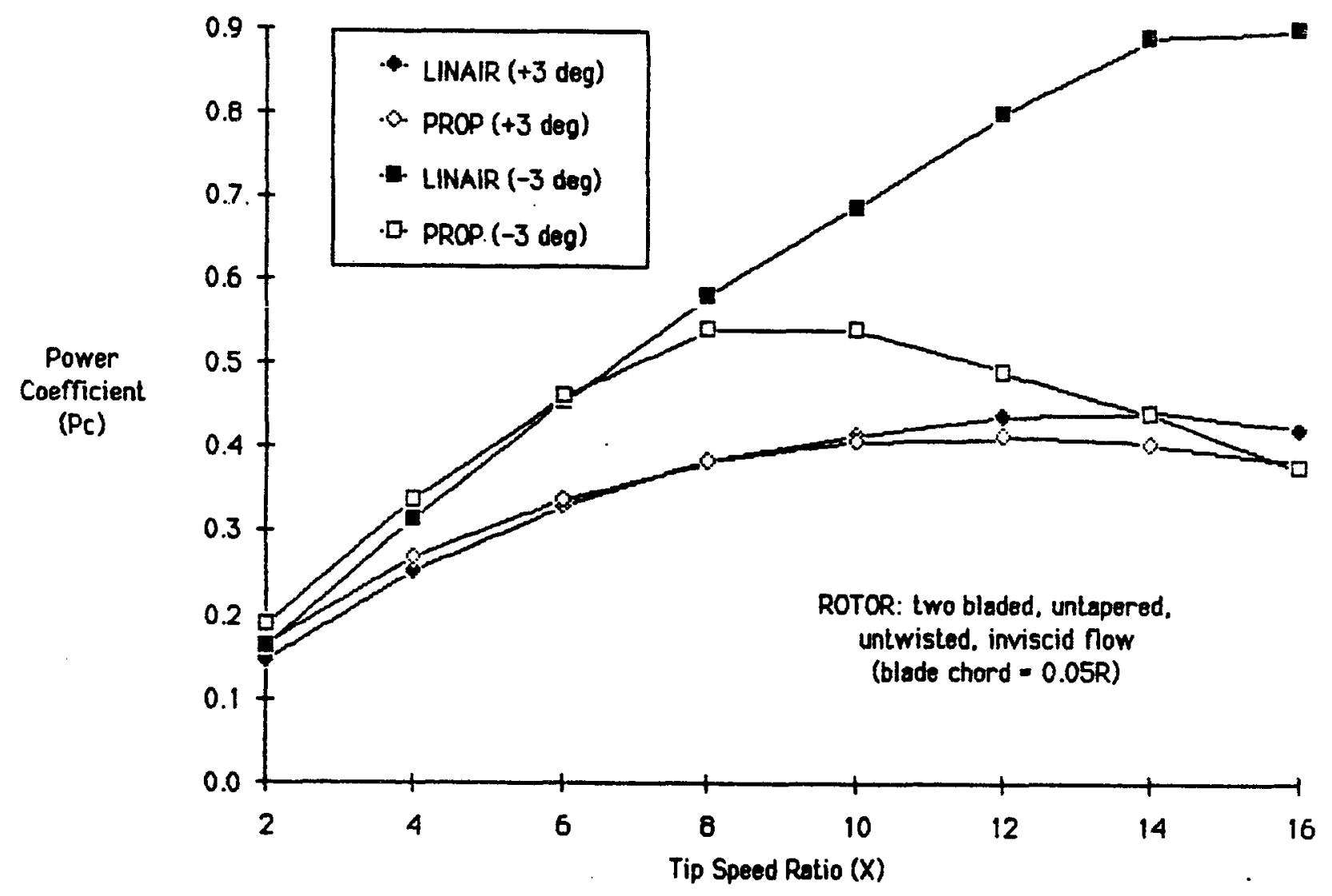

FIGURE 9. Comparison of PROP and LINAIR models at different pitch angle settings (rotor loadings) for a simple rotor -- power coefficient. 


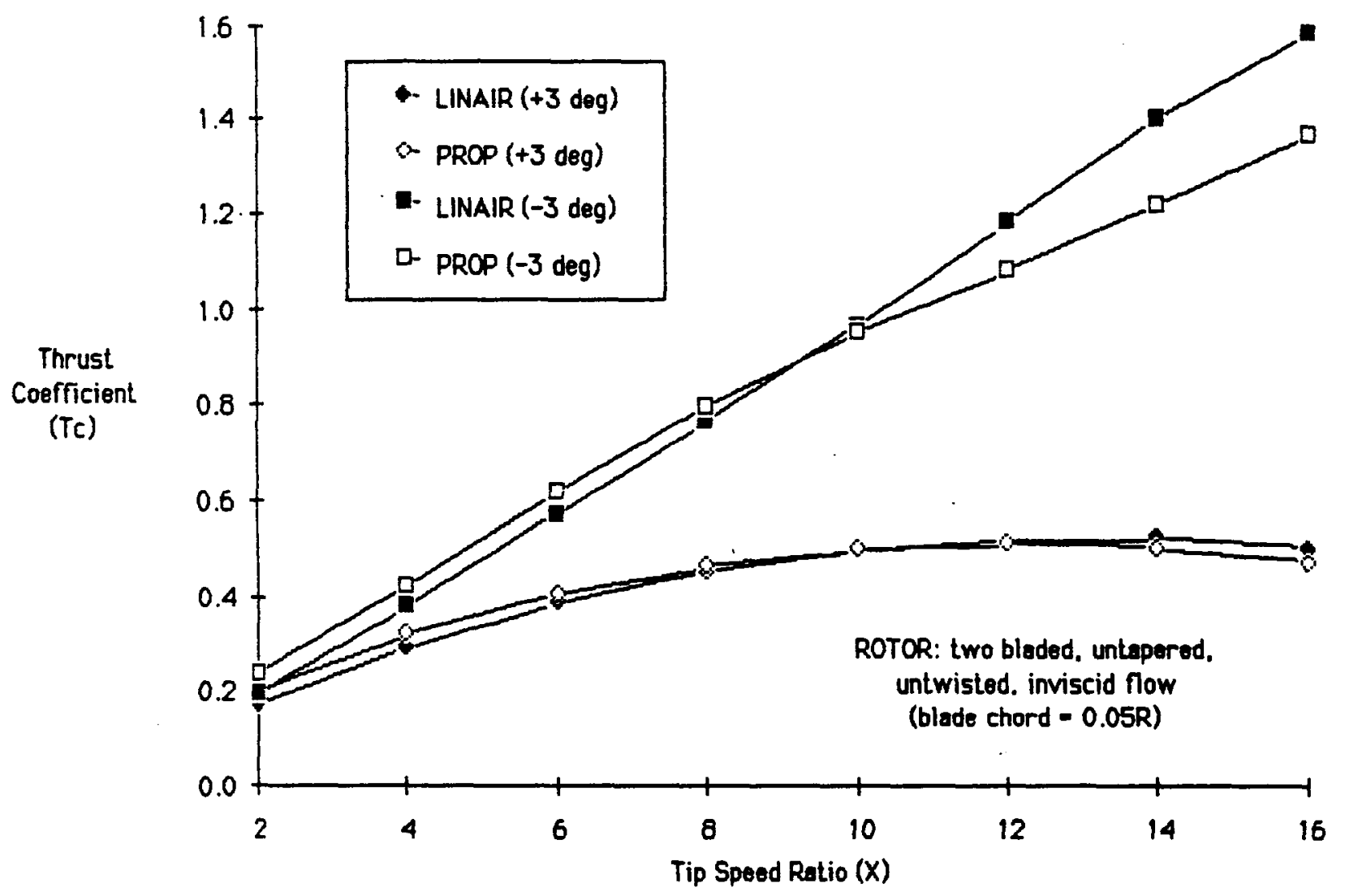

FIGURE 10. Comparison of PROP and LINAIR models at different pitch angle settings (rotor loa dings) for a simple rotor -- thrust coefficient. 
3) Ignoring variations in the wake vortex propagation speed and/or ignoring the wake expansion. In the model, the shed vorticity propagates downstream at the freestream speed as a cylindrical helix and does not itself experience any wake expansion or slowdown.

Reason 1) was discarded on the grounds that good agreement with PROP was observed for the lightly loaded rotor and on the basis of results from other test cases run by Kroo. The results of an investigation of reasons 2 ) and 3) are shown in Figure 11 again using a simple untapered, untwisted rotor. The solid line shows the power curve of the more heavily loaded rotor of. Figure 9 and was used as a baseline. It can be seen that neither doubling the wake length (the distance downstream to which the integration of the effects of the shed vorticity on the flow field at the rotor was taken) nor using a larger number of (i.e., smaller) legs for the integration along the vortex filaments made a significant difference to the predicted power curve. Therefore, reason 2) was discarded.

Kroo models each shed vortex filament as a cylindrical helix, each fliament being swept downstream at the free-stream speed. The filament paths can thus be visualized as a coil spring with the same, fixed number of turns per unit axial length. A highly, loaded rotor, however, causes considerable slowing of the flow passing through the rotor disk. Under such conditions, the shed vortex filaments are swept downstream at a lower speed, the swept speed.

Increasing the loading on the rotor should, therefore, be accompanied by an increase in the number of turns per unit length of Kroo's helix, the actual magnitude of the increase varying with radius. Figure 11 also shows the effect of a fixed compression of the helical vortex on the predictions of Kroo's model; two different swept speeds are shown. It should be noted that this modification is still not completely realistic since it takes no account of the increase in radius of the cylindrical helices which, by continuity considerations, would be associated with wake slowdown, nor does it take into account the variation in swept speed with tip speed ratio, and hence loading, nor does it maintain a constant wake length (i.e., in the model the wake gets shorter at higher loadings). As expected, the new wake model causes a greater interference at the blades, and thus lower power coeffi- 


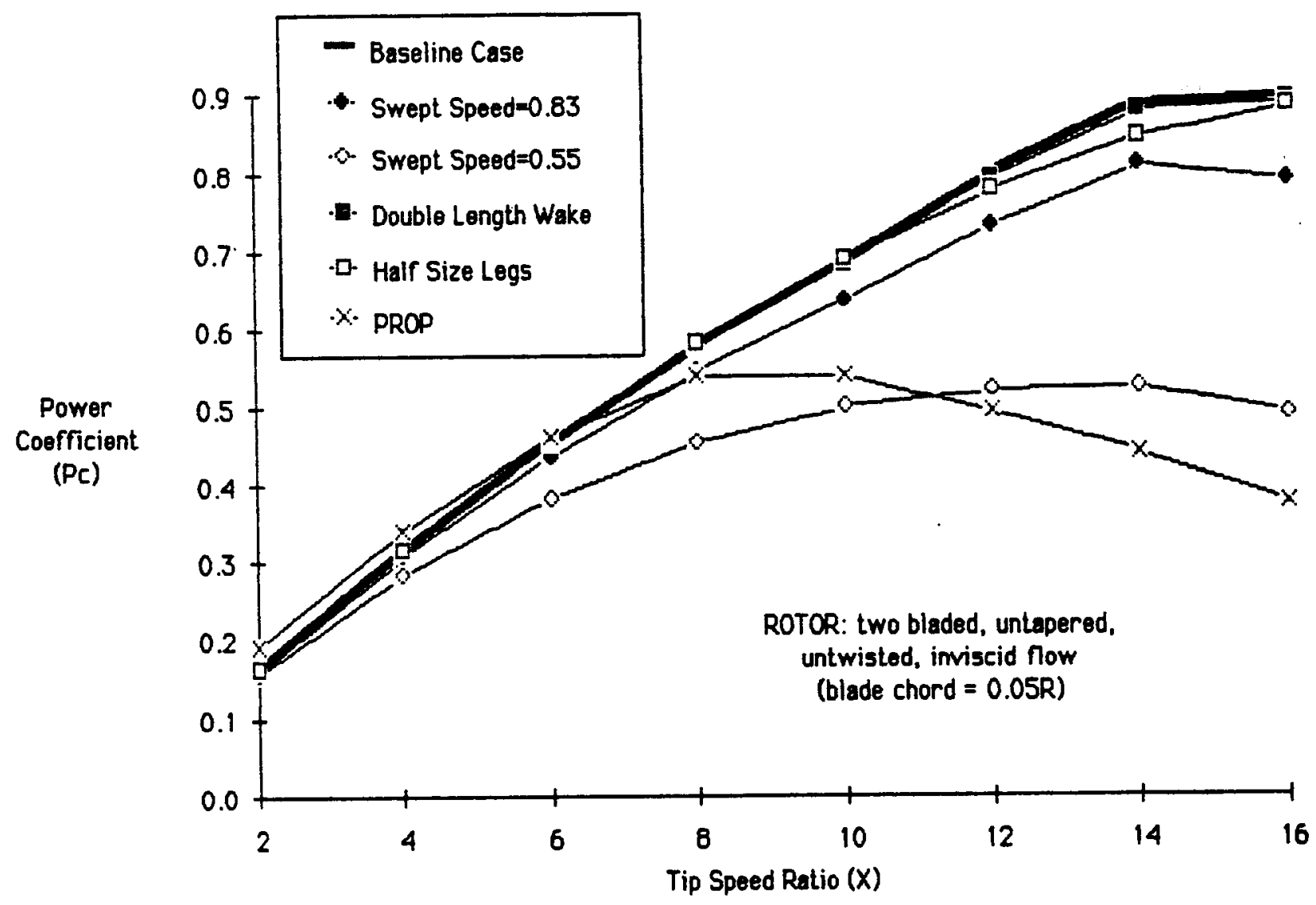

FIGURE 11. Effect of varying the wake geometry model used by LINAIR. 
cients, closer to PROP's predictions. Remaining differences were thought to be due to the remaining inadequacies in the wake model. Therefore, it was concluded that the differences between PROP and Kroo's model, which can be observed in Figures 9 and 10 , are mainly due to reason 3 ).

In reading this discussion, it should be remembered that Kroo's model was originally written to analyze aircraft configurations and no attempt was made to include the capability of accurately modeling the behavior of a wind turbine rotor.

Since it was beyond the scope of work to modify the code so that it would properly model the wake even under highly loaded conditions, it was decided to proceed with the design of tip devices with the existing version of Kroo's model for a more lightly loaded rotor than the Carter machine, and to make the assumption that these would still be reasonable designs for the real turbine. While it was recognized that this assumption was questionable, it appeared to be the only option in order to complete the design phase. Figure 12 shows the power curves predicted by PROP and Kroo's model LINAIR at a variety of feather angles for a linearized version of Carter's blades which were untwisted and untapered with a chord of 0.065 of the rotor radius. The $+4^{\circ}$ feather version was selected as the rotor for which tip devices would be designed.

Viscous drag effects were included using the function $C_{D}=0.01+k C_{L}{ }^{2}$ where $k=1 / 200$, giving a parabolic variation in drag coefficient similar to the NACA 23012 used at the tip of the Carter blade.

The total viscous drag, D, experienced by a tip device is given approximately by

$$
D=C_{D} 1 / 2 \rho(X v)^{2} A
$$

where $C_{D}$ is the mean dray coefficient, $\rho$ the air density, $X$ the tip speed ratio, $V$ the wind speed and $A$ the planform area. The power absorbed $P_{\text {lost }}$ by the tip device due to viscous effects is therefore 


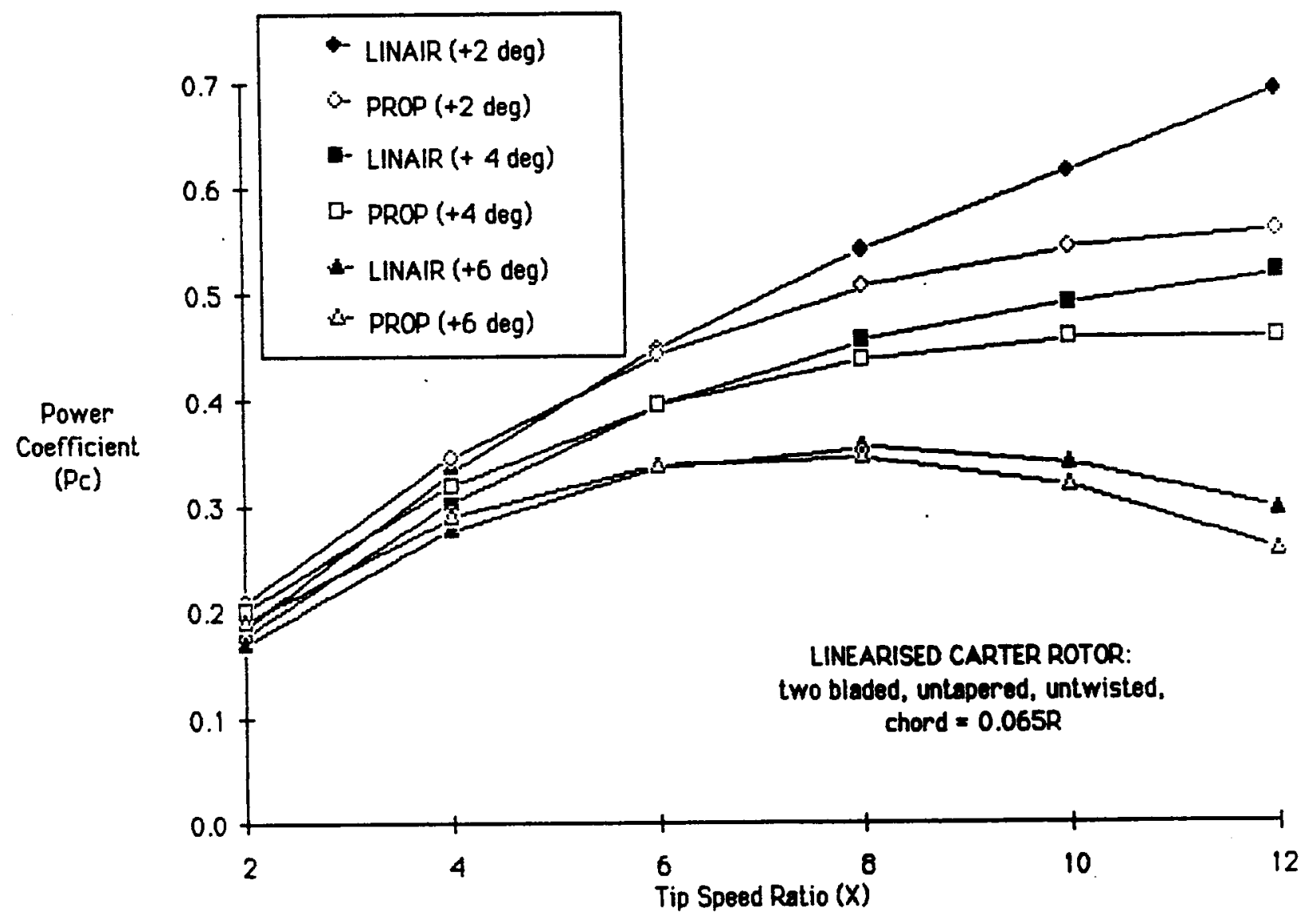

FIGURE 12. Comparison of PROP and LINAIR for a linearized version of Carter's blades at various feather angles. 


$$
\begin{aligned}
P_{\text {lost }} & =D \times v \\
& =C_{D} 1 / 2 \rho(x v)^{3} A
\end{aligned}
$$

Expressing this as a reduction in rotor power coefficient we obtain

$$
P_{C_{\text {lost }}}=C_{D} x^{3} A^{*}
$$

where $A^{*}$ is the planform area of the tip device normalized by the rotor swept area. For the Carter machine at a tip speed ratio of 10, a total tip area of $1.0 \mathrm{sq} f t$, and assuming a drag coefficient of 0.015 , this gives a value for $P_{C_{1}}$ of 0.02 , or about $5 \%$ of maximum $P_{c}$. Viscous losses on the tip devices are, therefore, significant, particularly at low wind speeds. Thus, it will be important to ensure that the tip devices are well streamlined and that the losses at the junction of the main blade and tip device due to interference and/or separation are minimized. Hoerner (1965) estimates the drag based on thickness $t$ and dynamic pressure $q$ of a "T" junction of two $44 \%$-thick struts to be given by $D=k_{t} q t^{2}$, where $k_{t}$ is in the range 3.3 for no fairing to 0.2 for an optimum fairing. Based on the thickness of the main blades, a range for $P_{C_{\text {lat }}}$ of about $0.03(7 \%)$ for the unfaired junction to $0.002(0.5 \%)$ for the ideally fairest case would be expected at $X=10$, assuming an " $L$ " junction has half the interference drag of a "T" junction. A bad fairing could, therefore, absorb all the extra power gained from the effects of the tip devices, whereas a properly designed fairing will result in a minimum performance loss.

\section{DESIGN}

Complete specification of a simple one element tip device involves six parameters -- the airfoil, span, aspect ratio, taper, twist, and cant angle. An exhaustive computerized optimization of all these variables was neither practical nor necessary. It should be noted that Kroo's model is approximate, and is known not to properly account for the onset of the brake state. Therefore, designs were optimized using a practical, human-controlled approach taking as a starting point the parameter values used in successful tip devices for wings. In this way it was expected that maximum advantage could be taken of previous research work. 
A restriction was applied during design that the projected radius of the complete system (main blade and tip devices) should not exceed the original projected radius of the Carter rotor. Although a power increase can always be realized by a simple tip extension, this also increases the gale loads experienced by the blade roots due to the increased projected area and moment arm. One of the main objectives of using tip devices is to realize a power increase without a signficant load penalty.

The design procedure was as follows. An initial design was first selected on the basis of previous studies and analyzed. Then small variations in one or two of the defining parameters listed above were made and the resulting designs analyzed. Those designs that gave improved performance were selected for further analysis while those which showed a performance decrease were rejected. Variations that resulted in an improvement were further varied until a maximum was reached. For example, if an increase in aspect ratio improved performance, this parameter would be increased further until the performance was maximized with respect to that parameter. Then another parameter was altered and the process repeated.

The procedure continues until no further improvement occurs. It was usually necessary to iterate several times before reaching a satisfactory design.

Sometimes a parameter could not be varied beyond a certain point due to one of several design constraints, such as tiplet span or maximum lift coefficient. In addition, sometimes it was necessary to vary two variables such as chord and incidence together in order to realize a performance improvement. At times it was desirable to try a wide range of values for a single variable in order to see if there was more than one design with high performance.

This approach generally results in designs that are rapidly derived, close to optimal and satisfactory for this early stage of wind turbine tip device development.

The range of values used for the successful tip devices discussed in the introduction is somewhat limited. Typically, the planform is tapered with the root 
chord equal to or about half the tip chord of the main wing, and a tip chord that is quite small compared to the chord of the main wing. The span typically varies from some what greater than the main wing chord to a small fraction of it. The twist is typically small or zero. The devices are usually mounted at zero incidence relative to the chord of the main blade. These data were used to define starting configurations for the tip device design optimization.

The tilt angles used have ranged from -90 degrees (in the pressure direction) to +110 degrees (in the suction direction and canted in towards root). Tip devices mounted at a tilt angle of zero are simply wing extensions. All the upwind tip devices analyzed had lower predicted performance than that of the plain rotor. Appendix B contains a comprehensive listing in both tabular and graphical form of all the confirmations analyzed and their predicted performance.

Virtually all devices require some fairing with the main wing in order to minimize viscous losses. The most typical fairing method is to simply bend the main wing into the tip device, resulting in a smooth transition. In the case of the vortex diffuser vane, the device is mounted behind the main wing, virtually eliminating the fairing problem. While multielement devices such as the wing tip sails have been reported to perform better than single-element devices on a per-area basis; they are also more susceptible to viscous loss problems because of the difficulty of fairing them into the main blade. It should be noted that Kroo's code cannot predict separation and a multielement design will therefore have a high risk associated with it in terms of achieving predicted performance.

The geometrical dimensions of the three tip designs selected for field tests are defined in Figure 13, specified in Table 2 and shown in Figure 14. The expected performance improvement is shown in Figures 15 and 16 . In each case, the performance improvement increases with wind speed. The single-element device has the largest effect overall; the shark's fin tip shows a small but consistent performance improvement on the order of $0.3 \mathrm{~kW}$; the double element device lies somewhere between these two. The single element device is in fact quite similar to the successful shapes used on nonrotating wings. 

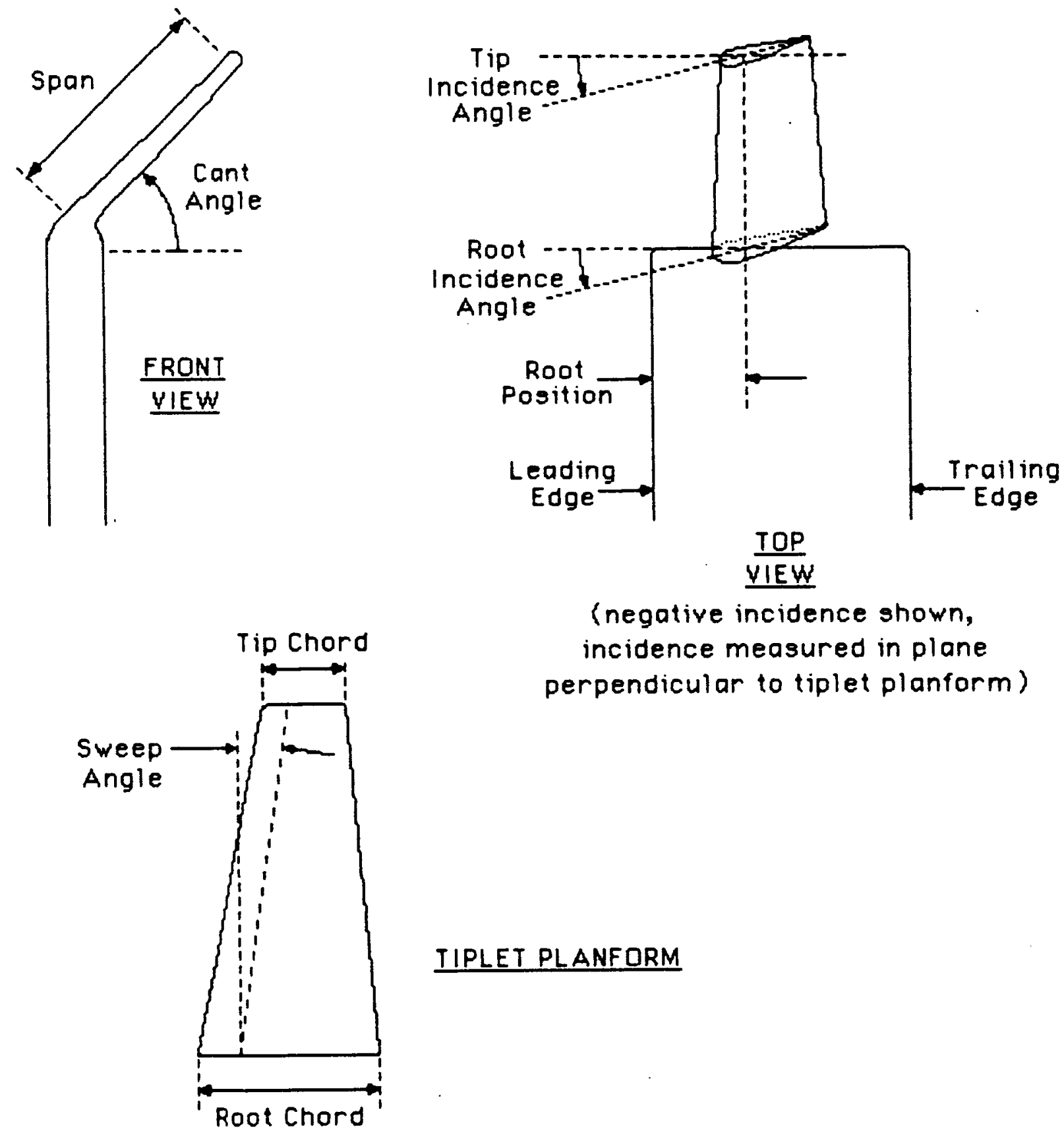

\section{TIPLET PLANFORM}

FIGURE 13. Geometric parameters defining tip devices. 
TABLE 2. Specifications of tip devices recommended for testing.

\begin{tabular}{|l|r|c|c|c|c|c|c|c|}
\hline $\begin{array}{c}\text { Tiplet } \\
\text { Design }\end{array}$ & $\begin{array}{c}\text { Root } \\
\text { Posi- } \\
\text { tion } \\
\%\end{array}$ & $\begin{array}{c}\text { Norma- } \\
\text { lized } \\
\text { Span }\end{array}$ & $\begin{array}{c}\text { Norma- } \\
\text { lized } \\
\text { Root } \\
\text { Chord }\end{array}$ & $\begin{array}{c}\text { Norma- } \\
\text { lized } \\
\text { Tip } \\
\text { Chord }\end{array}$ & $\begin{array}{c}\text { Root } \\
\text { Inci- } \\
\text { dence } \\
\text { Deg. }\end{array}$ & $\begin{array}{c}\text { Tip } \\
\text { Inci- } \\
\text { dence } \\
\text { Deg. }\end{array}$ & $\begin{array}{c}\text { Sweep } \\
\text { Deg. }\end{array}$ & $\begin{array}{c}\text { Cant } \\
\text { Deg. }\end{array}$ \\
\hline $\begin{array}{l}\text { "Shark fin" } \\
\text { Single element }\end{array}$ & 25 & 0.065 & 0.065 & 0.0325 & 3 & 0 & 30 & 90 \\
$\begin{array}{c}\text { Double element } \\
\text { Surface 1 }\end{array}$ & 50 & 0.05 & 0.0216 & 0.0108 & -2 & -6 & 0 & 4 \\
Surface 2 & 31 & 0.056 & 0.0162 & 0.0162 & 2 & 2 & 0 & 45 \\
\hline
\end{tabular}

NOTE: Dimensions normalized by rotor radius. Root position is given as a percentage of the tip chord of the main blade. 

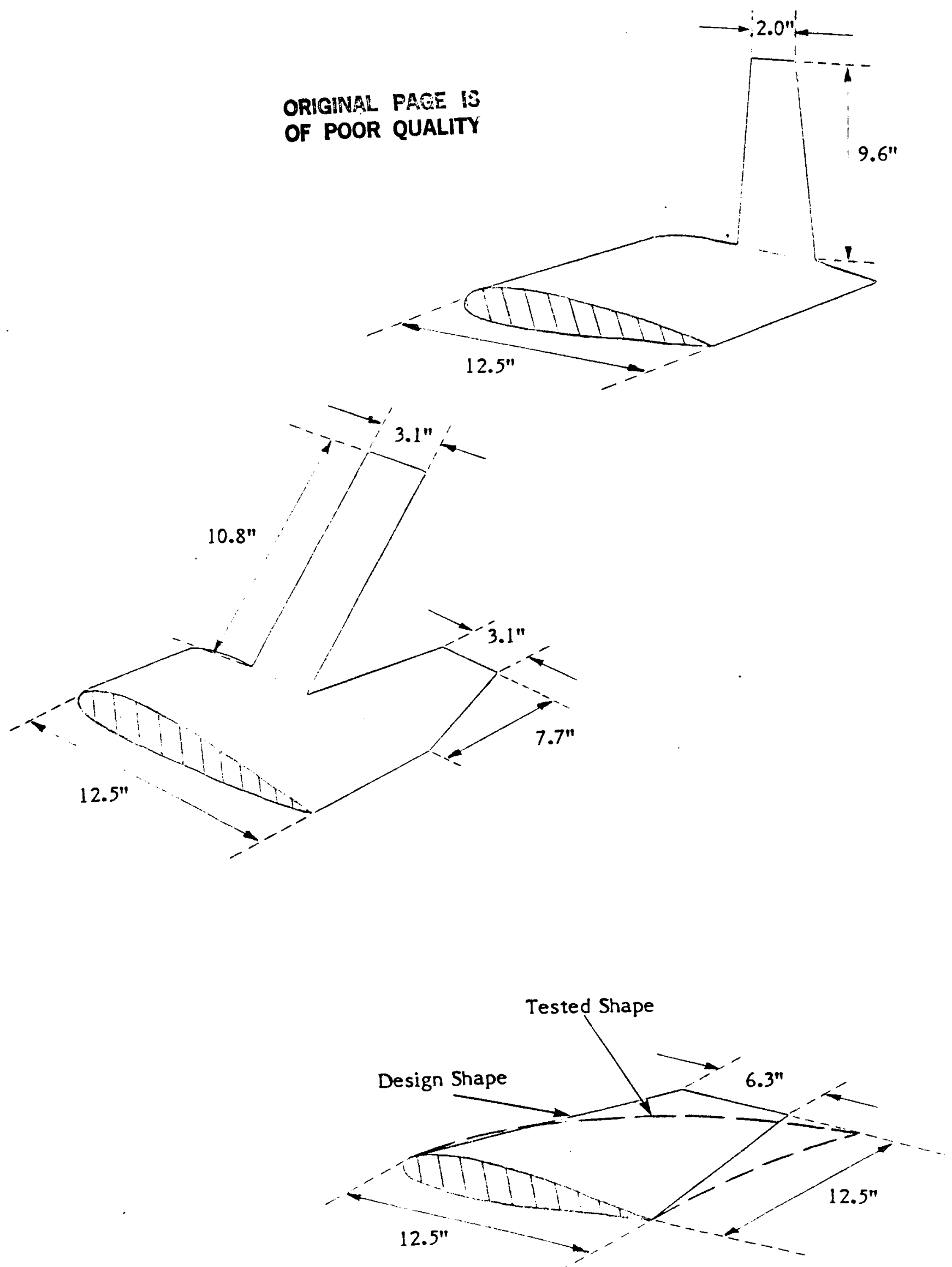

FIGURE 14. Basic dimensions of tip devices. 


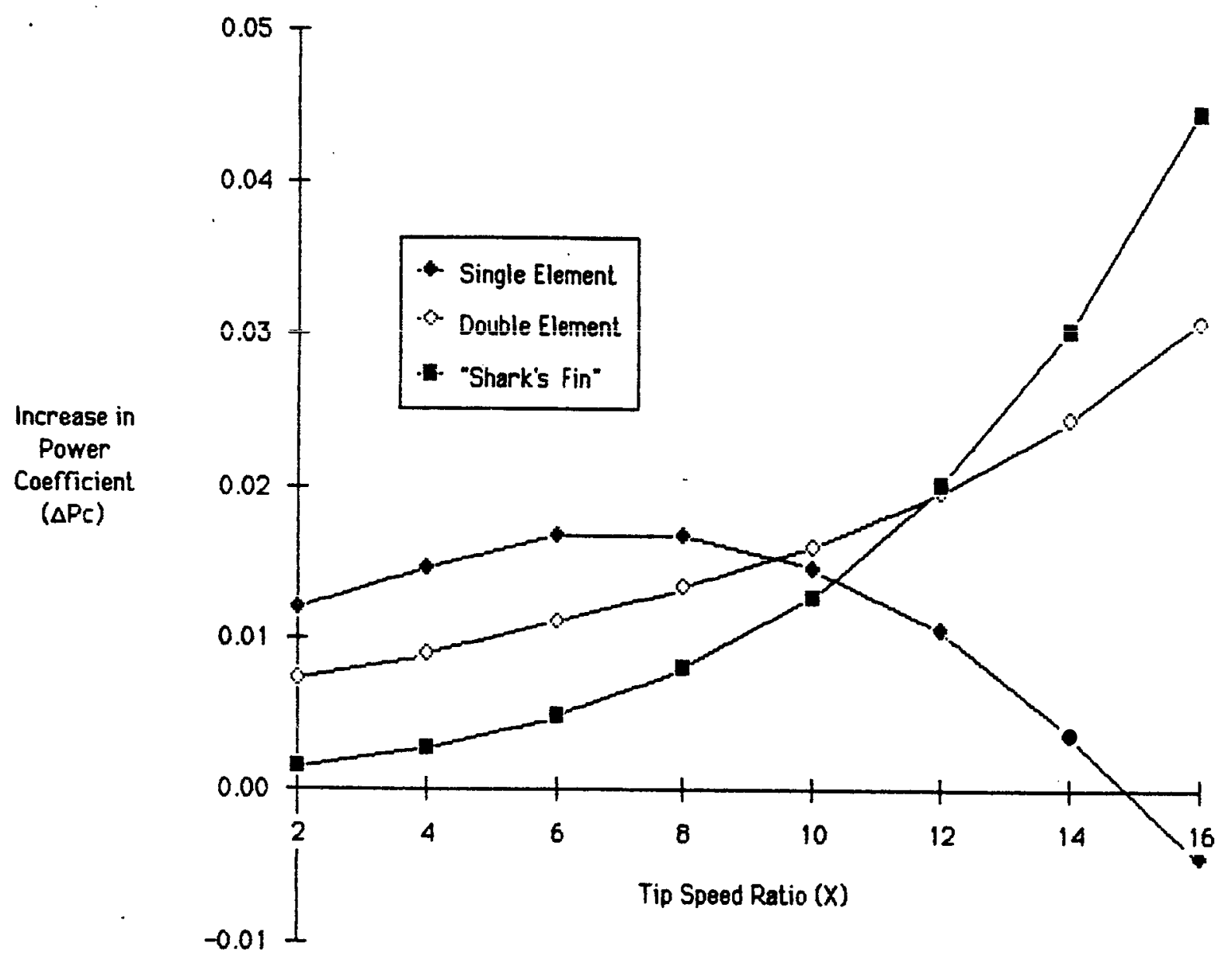

FIGURE 15. Predicted performance improvement using tip devices ( $\triangle \mathrm{PC}$ vs. $\mathrm{X}$ ). 


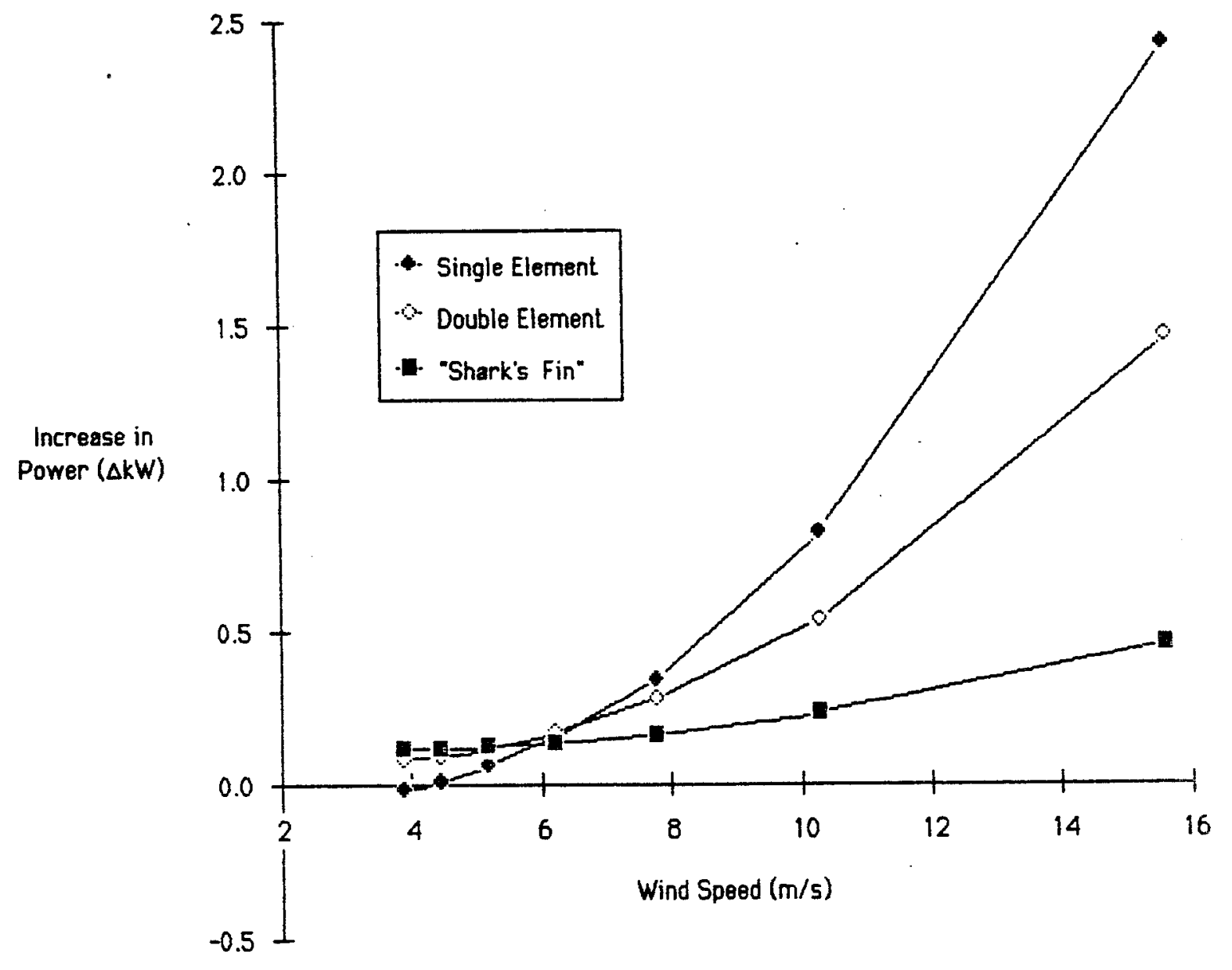

FIGURE 16. Predicted performance improvement using tip devices ( $\Delta \mathrm{P}$ vs. $\mathrm{V}$ ). 
The tips were chosen to cover the basic range of possible configurations; while the shark's fin represents a simple change in tip shape, the single-element and multi-element designs represent increasingly complex advanced tip devices.

It is of interest to compare the predicted performance improvement with tip devices to that produced by a simple blade extension. The effectiveness of the single element tip device will be taken as an example. The single-element device is predicted to give a net improvement in maximum power coefficient of about $4 \%$. The device has a normalized span (based on rotor radius) of 0.05 and a normalized mean chord of 0.016 . If the equivalent area were used as a simple constant chord extension to the main blade, the expected increase in power coefficient would be only about $2.5 \%$ based on original rotor area, with an increase in gale loads because of the longer rotor arm and greater projected area. However, at wind speeds around cut-in, the single element device is actually predicted to reduce performance slightly.

It should be noted that Kroo's code is a vortex line model and may not be a reliable performance predictor for the shark's fin tip which has a very low aspect ratio. This tip was included in the test to represent a simple change in tip shape.

\section{FABRICATION}

For the field test phase a new pair of Carter blades were purchased and modified to accept replaceable "plug-in" tips. A 12 " section of the original tip of each blade was first removed and two aluminum tubes bonded to the inside of the skin of the remainder to form female mounting sockets for the various tips, a large diameter tube at the $1 / 4$ chord point to carry most of the loads, and a smaller tube near the trailing edge to act as a locating pin. The removed sections were fitted with two matching male plugs to become the "baseline" tips and represent the original, unmodified Carter blades. A hole was drilled through the larger tubes perpendicular to their axis to accept a shear pin to carry the centrifugal loads. Figure 17 shows a closeup of the tip attachment pins on one of the tip devices. The single-element Whitcomb-type tip and the double-element Spillman-type tip were made from foam and fiberglass wing sections. The foam, used to fix the basic 
ORIGINAL PAGE IS

OF POOR QUALITY

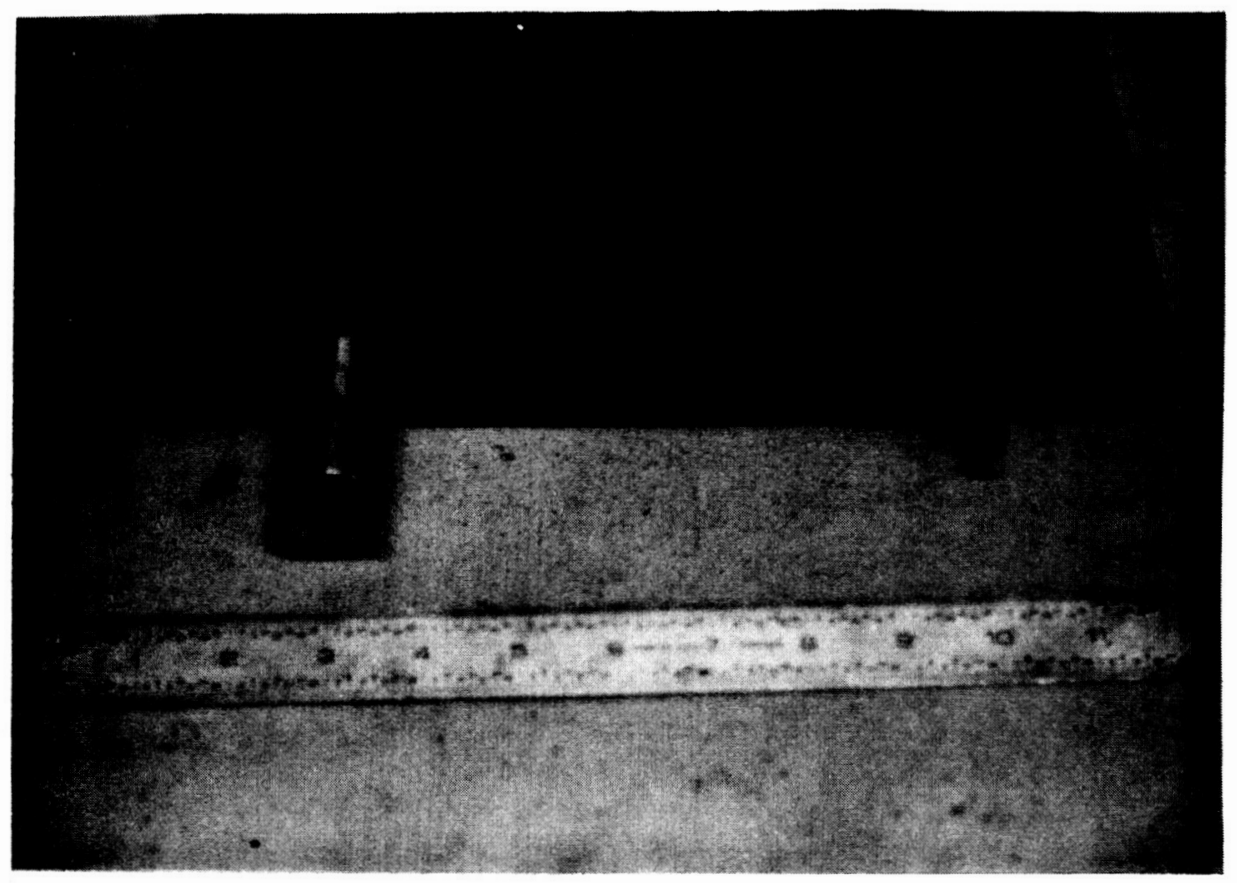

FIGURE 17. Closeup of tip attachment pins in an unfinished tip. 
dimensions, was first cut out using a hot wire stretched between templates of the airfoil sections (Figure 18) and it was then covered with a glass fiber and epoxy skin for strength. The individual wing sections were then joined together, fitted with attachment plugs and finished to produce a complete tip (Figure 19).

Structural calculations were made to determine the necessary sizes for the various parts and some structural tests were made on the finished tips to ensure their integrity. The tips would be subject to two types of loads, centrifugal and aerodynamic. Centrifugal loads would amount to about $80 \mathrm{~g}$ s. Since each tip weighed about four pounds, tests to ensure their ability to withstand centrifugal loads would require the construction of a substantial test rig capable of developing loads of over $300 \mathrm{lbs}$ and were not carried out. The aerodynamic forces were considerably smaller however, and easier to simulate; tests were therefore performed to ensure the strength of the tip elements in this loading mode.

The shark's fin tip, because of its highly nonlinear shape, was made by first carving a wooden plug, making a female mold from the plug, and then laying up a glass fiber and epoxy skin in the mold (Figure 20).

Each tip had an aluminum tube mounted at the leading edge which could be filled with lead shot to balance the tip about its quarter chord point.

During the field tests, the slight gap at the junction between the main blade and the tip device was sealed with thin adhesive tape to prevent through flow.

When the tips had been finished, flow visualization tests were carried out in rectilinear flow to determine whether fairings were required at the intersection of the wing elements to prevent separation. This was done in calm air on a dry lake bed by mounting the tips out to the side of a pick-up truck and observing the behavior of cotton tufts at various angles of attack and sweep at $55 \mathrm{mph}$. Whilst it was recognized that this type of test is only a crude representation of real operating conditions it was carried out in an attempt to avoid major regions of separation on the tips. In addition, since the real tip speed of the rotor would be almost $140 \mathrm{mph}$, it was reasonable to assume that separation tendencies would be reduced in the tests on the actual turbine. 


\section{ORIGINAL PAGE IS \\ OF. POOR QUALITY}

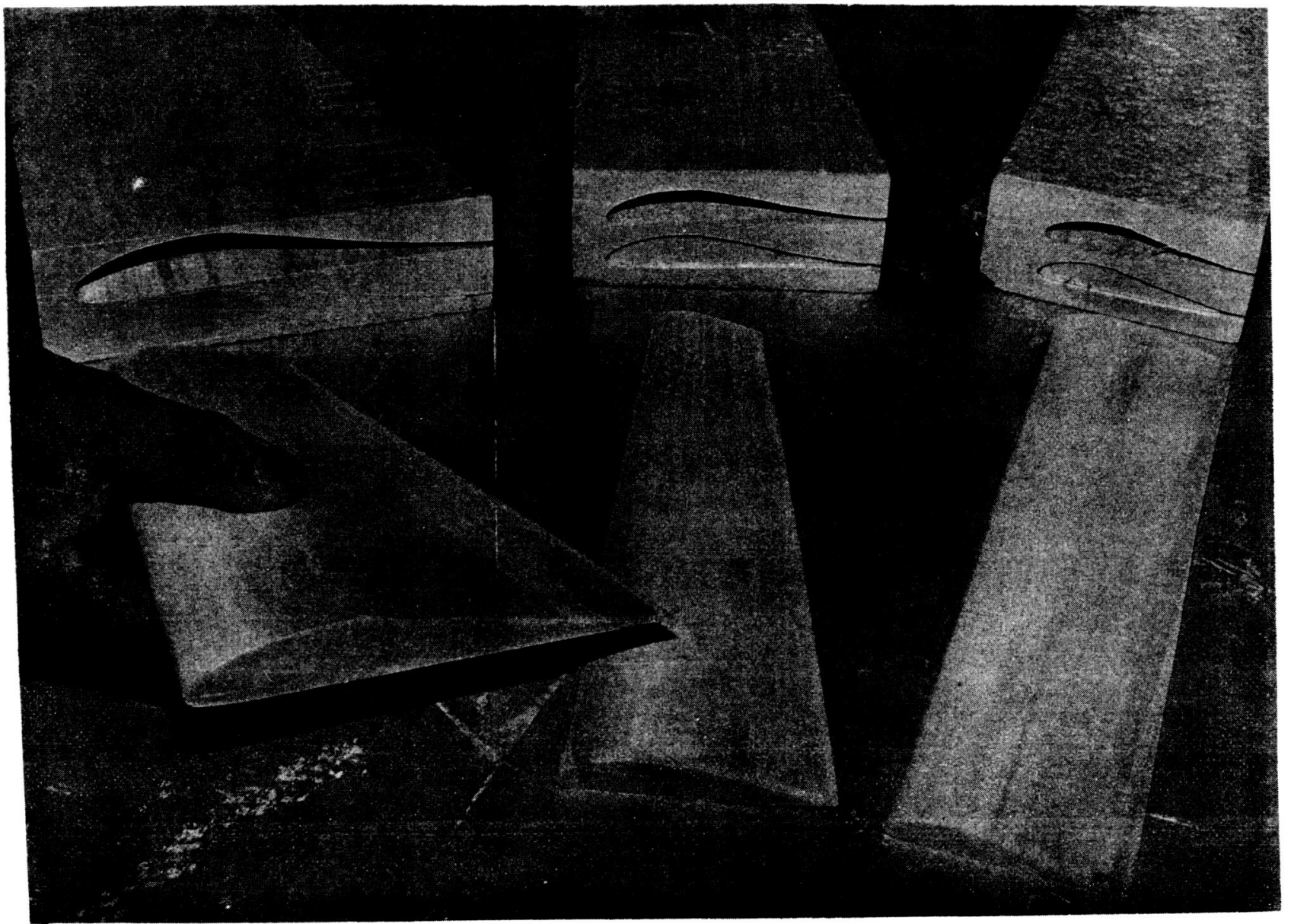

FIGURE 18. Foam cores for tip devices "hot-wired" from blocks. (From left to right: double element surface 2 , single element, double element surface 1.) 


\section{ORIGWAL PAGE IS \\ OF POOR QUALITY}

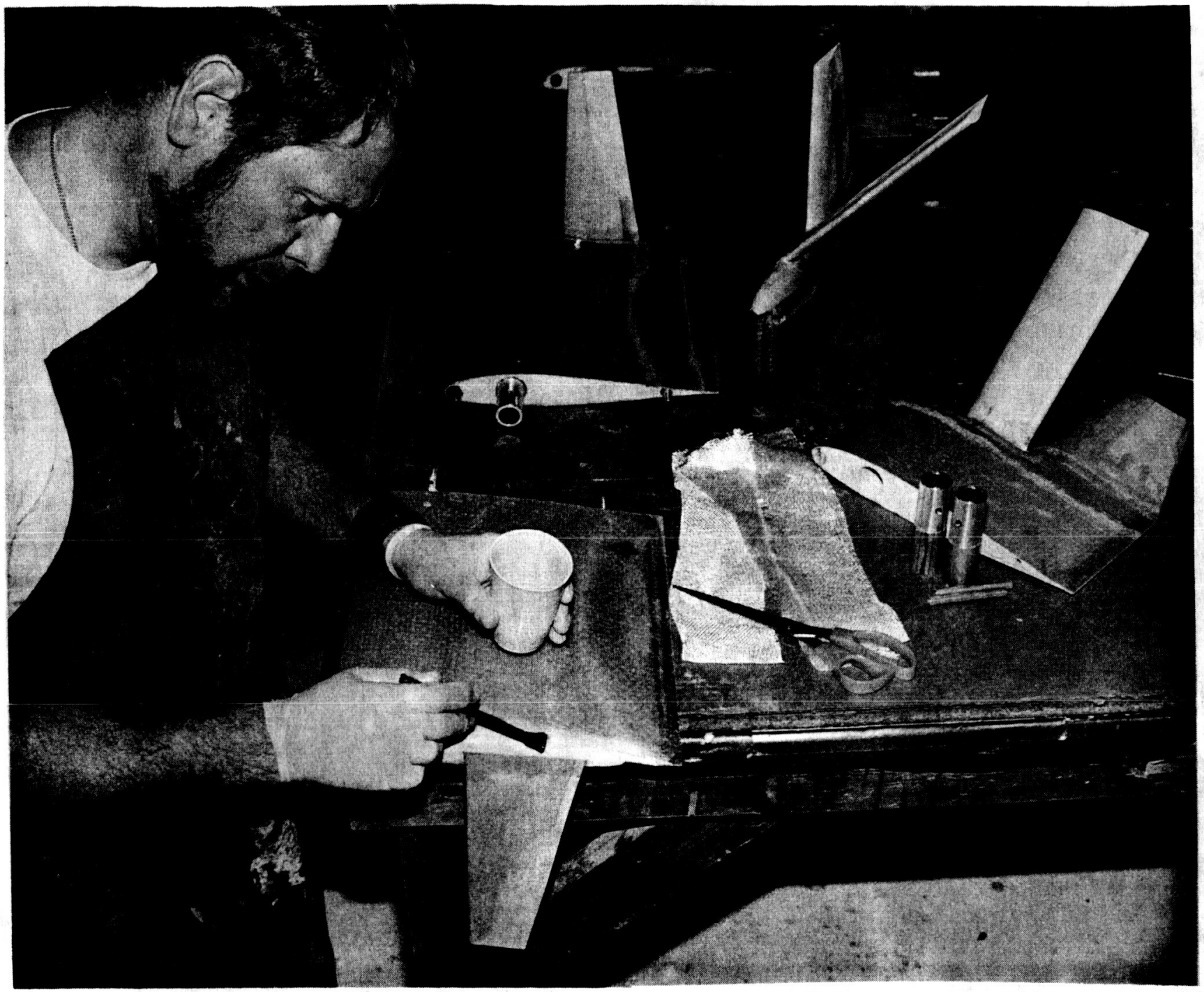

FIGURE 19. Attachment of tip elements to tip body. 


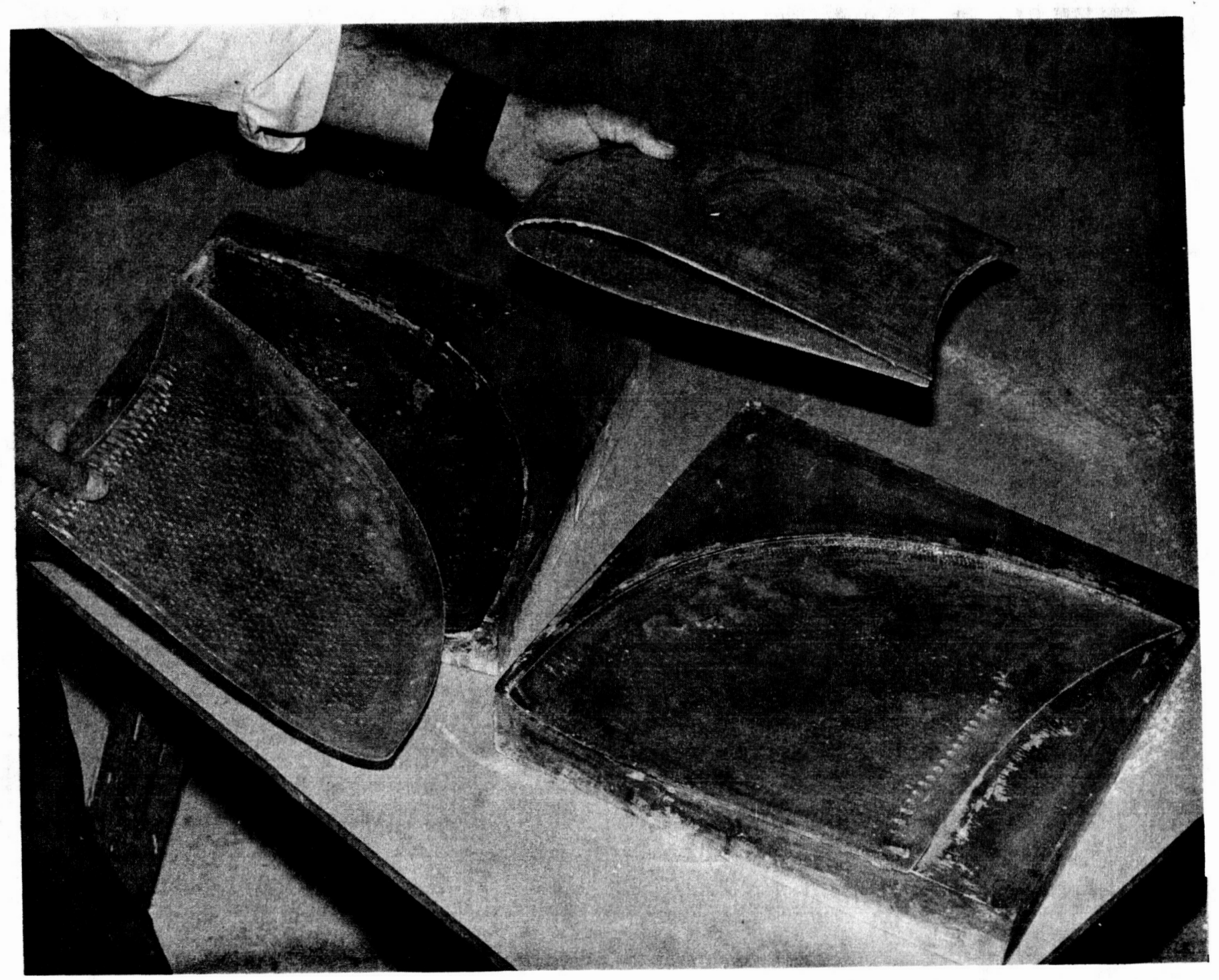

FIGURE 20. Unfinished shell sections for shark's fin tips showing molds from which they were released. 
Premature stall was observed on all the airfoils during the tuft tests; at least part of the reason for this was attributed to the low Reynolds numbers involved. It was found that tip strips of about $0.004^{\prime \prime}$ thickness at the $25 \%$ chord point improved this condition significantly. At substall angles of attack, the tests showed attached flow over most of the tips; however, they indicated that a single small fillet was required at the junction of element No. 2 and the main blade of the double-element device. The flow at the junction of the single-element device stayed attached until the element itself separated. Clay was used to determine the minimum fairing shape necessary for the double element device. This could then be reproduced later back in the shop using more permanent methods. The required fairing was quite small.

Following the addition of the required fillet, the pair of blades, the four pairs of tips, and the hub were shipped back to Carter Wind Systems for static balancing and final surface finishing before installation on the test turbine. The three finished tip devices are shown in Figure 21.

\section{RESULTS}

The field test was carried out during the period from August to October 1984 on one of the $25 \mathrm{kw}$ wind turbines manufactured by Carter Wind Systems of Burkburnett, Texas, in a windfarm managed by San Gorgonio Farms Inc. in San Gorgonio Pass, near Palm Springs, California. The terrain is quite flat with a slight slope downwards to the south-southeast. The turbine selected was at the extreme southwest corner of the array. Since the prevailing wind direction is west, this would avoid wake interference from other turbines as much as possible and keep out of the way of access roads and maintenance operations. A photograph of the field test site is shown in Figure 22, the test turbine is nearest the camera in the center of the picture.

Each tip device pair was tested in turn for about two weeks. The amount of performance data collected for each configuration is shown in Table 3. The performance curve for the baseline configuration is shown in Figure 23 and 24 . Figures 25 , through 30 show the measured power curves using each of the tip devices. The method for data collection and reduction is described below. 


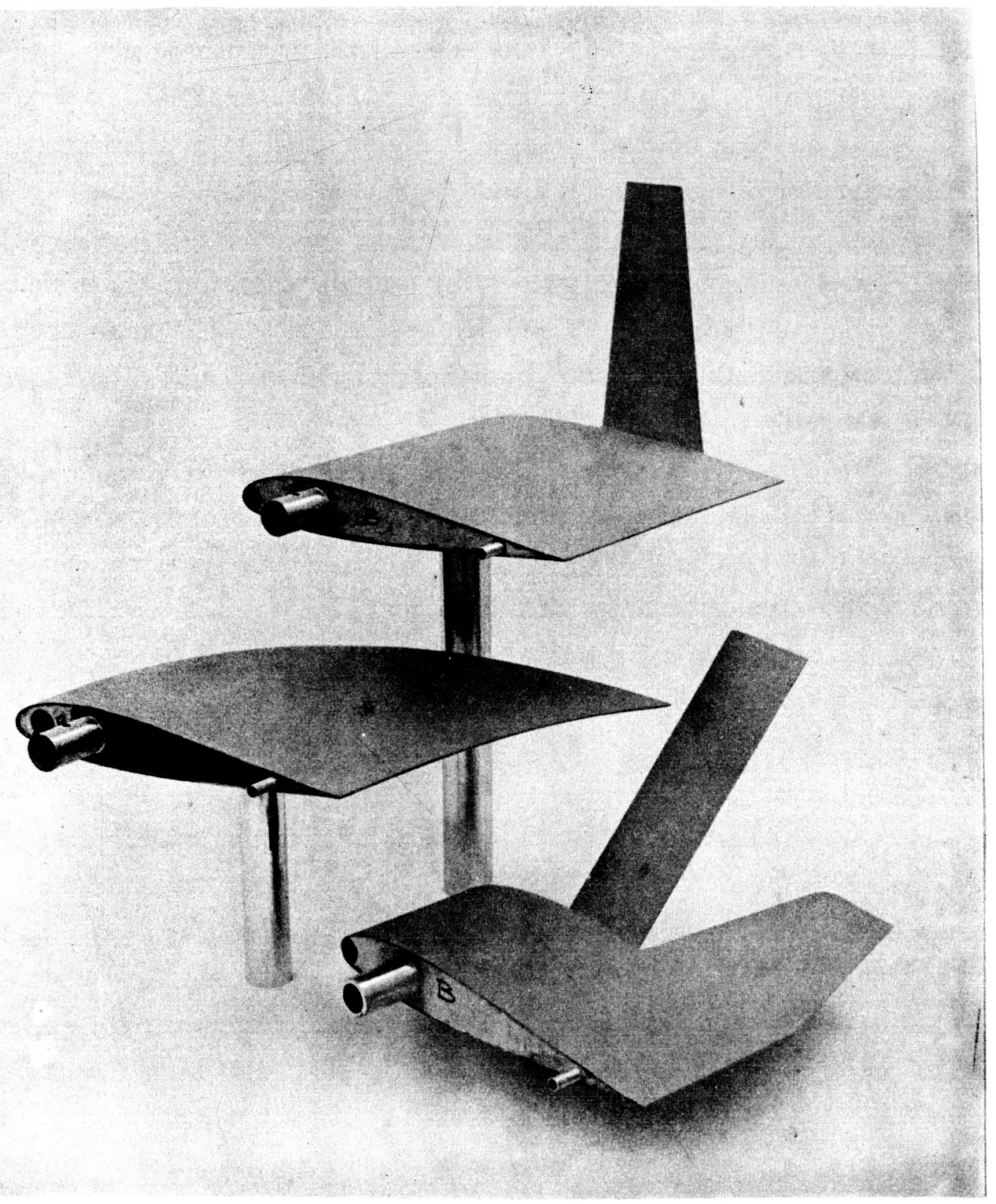

FIGURE 21. Finished tip devices. 


\section{ORIGINAL PAGE IS}

OF POOR QUALITY

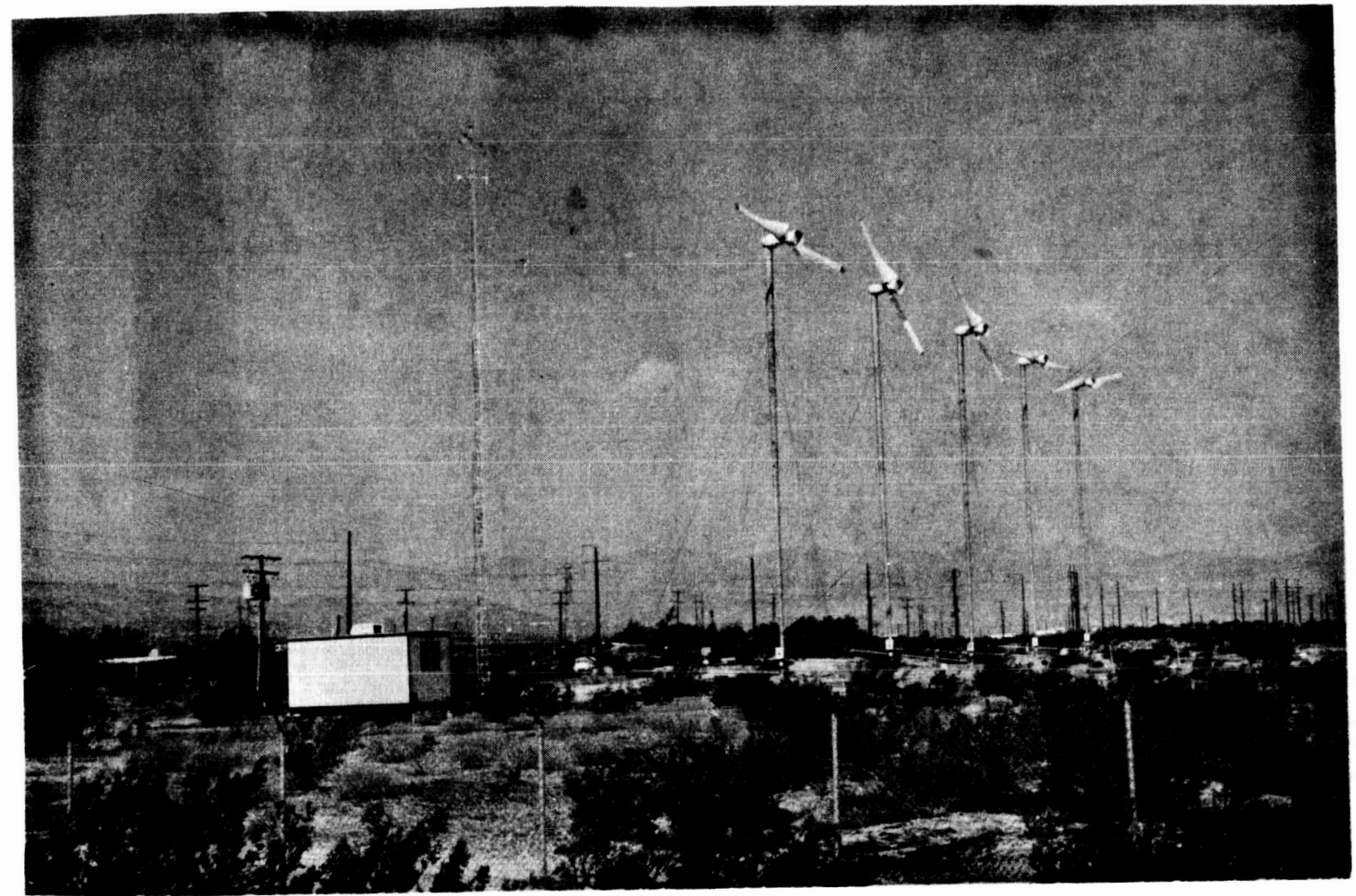

FIGURE 22. Field test site looking northwest. 
TABLE 3. Amount of performance data collected for each tip device.

\begin{tabular}{|l|r|r|}
\hline \multirow{2}{*}{ Tip Device } & \multicolumn{2}{|c|}{ Amount of Data } \\
\cline { 2 - 3 } & Readings & Hours \\
\hline Baseline & 163,900 & 109.3 \\
Shark's Fin & 70,700 & 47.1 \\
Single Element & 40,500 & 27.0 \\
Double Element & 127,900 & 85.3 \\
\hline
\end{tabular}




\section{ORIGINAL PAGE IS
OF POOR QUALITY}

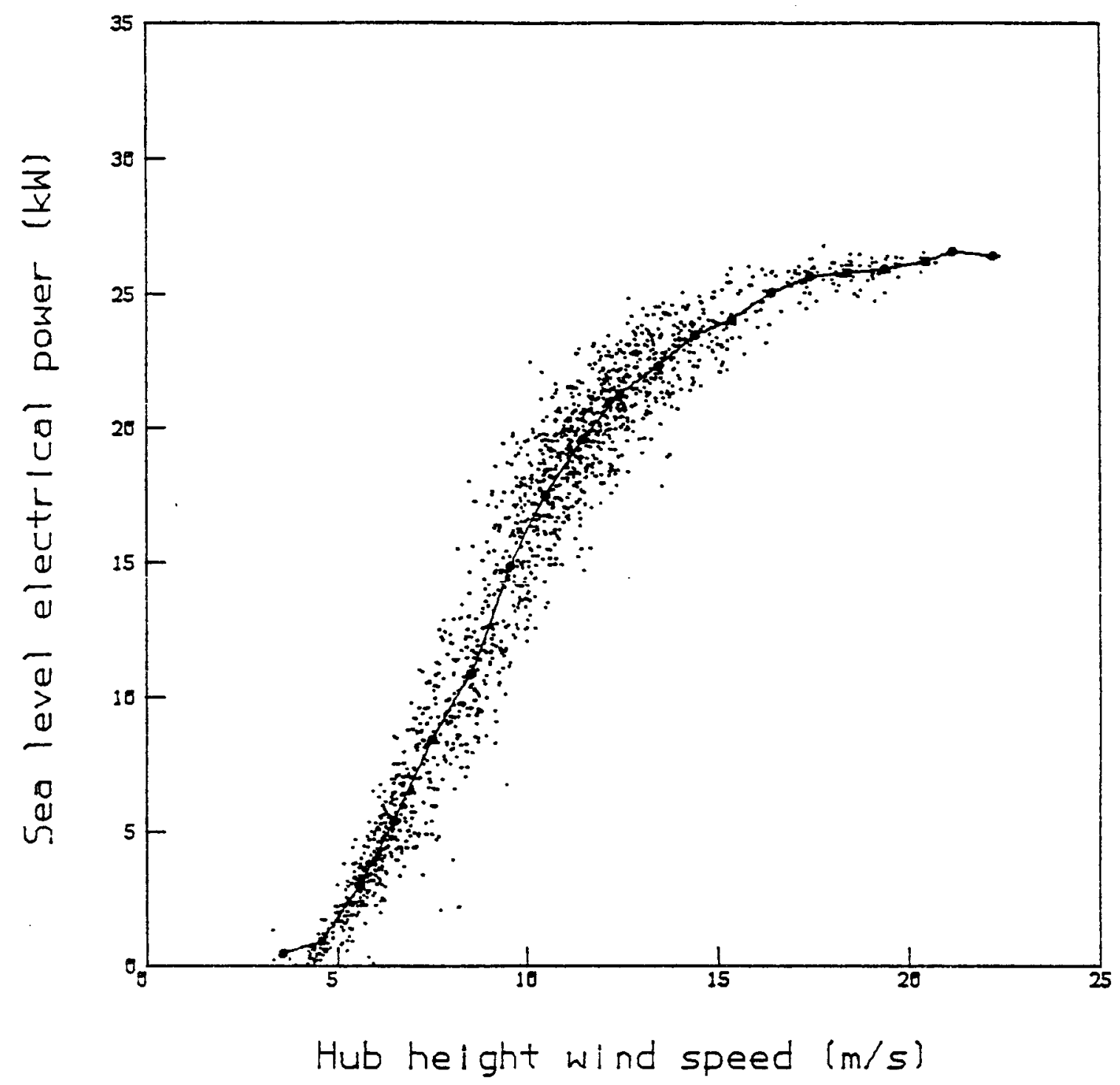

FIGURE 23. Scatter of four-minute average values for rotor with baseline tips. 


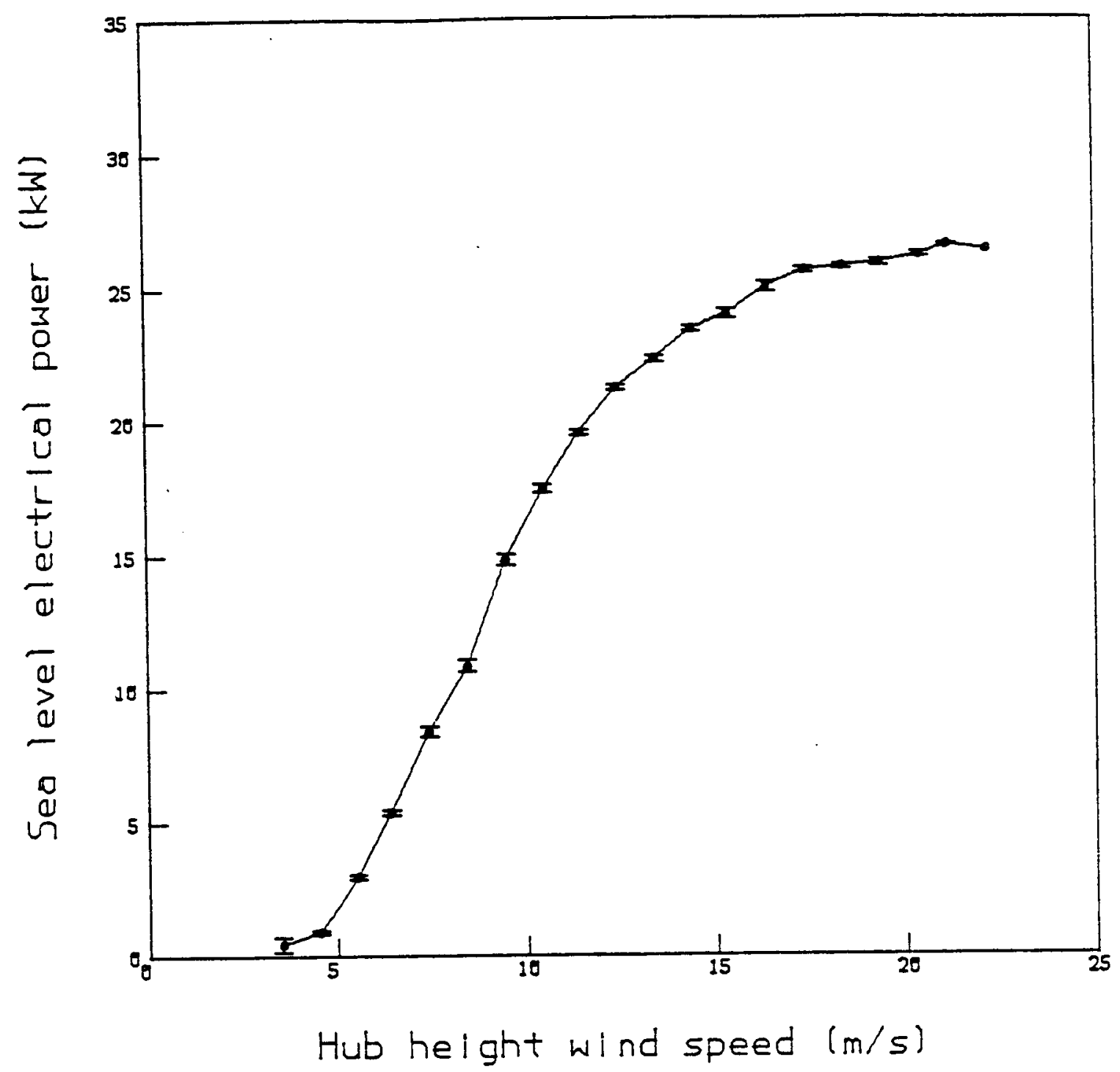

FIGURE 24. Results of bin sort of four-minute averages for rotor with baseline tips. 
A Compaq portable computer with a multi-channel 12-bit A-D converter was used to take field data, power, three components of wind speed, air temperature and pressure being recorded. A calibrated watt transducer installed in the control box at the base of the turbine provided a $0-1$ volt DC signal proportional to the turbine power output and an aspirated temperature transducer mounted at hub height recorded the ambient temperature. The UVW anemometer and the temperature sensor were mounted on an 80-foot portable crank-up tower. The pressure transducer was mounted inside the A-D converter at ground level. This 80-foot altitude difference represents an almost constant offset in the pressure value of about $+3 \mathrm{mb}(\sim 0.25 \%)$ and can be neglected.

Air temperature and pressure measurements were necessary in order to determine the air density; at the test site in the summer, daytime temperatures may reach $110^{\circ} \mathrm{F}$ while at night the air can cool to about $60^{\circ} \mathrm{F}$. At constant pressure, this temperature change represents a density change of about $10 \%$ which could mask any differences in power output produced by the tip devices. The biggest change in pressure that could be expected over the duration of the test would be about $1 \%$ and normally changes are much less than this in a 24-hour period unless a storm front passes through. Humidity changes were expected to affect the air density by less than $1 \%$ and were therefore neglected.

A software package was written to make it possible to monitor a plot of the power curve, based on either bin sorts or polynomial curve fitting, as it developed, making it easier to identify when sufficient data had been collected for each configuration. The raw data were also stored for future reference and postprocessing.

The data acquisition system ran unattended in the small air conditioned trailer which can be seen in Figure 22. The trailer and tower were set up about $130 \mathrm{ft}$ to the south of the turbine. The air conditioner and the electronics ran from two separate transformers connected to the $480 \mathrm{~V}$ lines to avoid data loss due to a drop in line voltage during cycling of the compressor.

The A-D converter scanned all six data channels (ambient pressure, ambient temperature, three components of wind speed and electrical power) approximately 


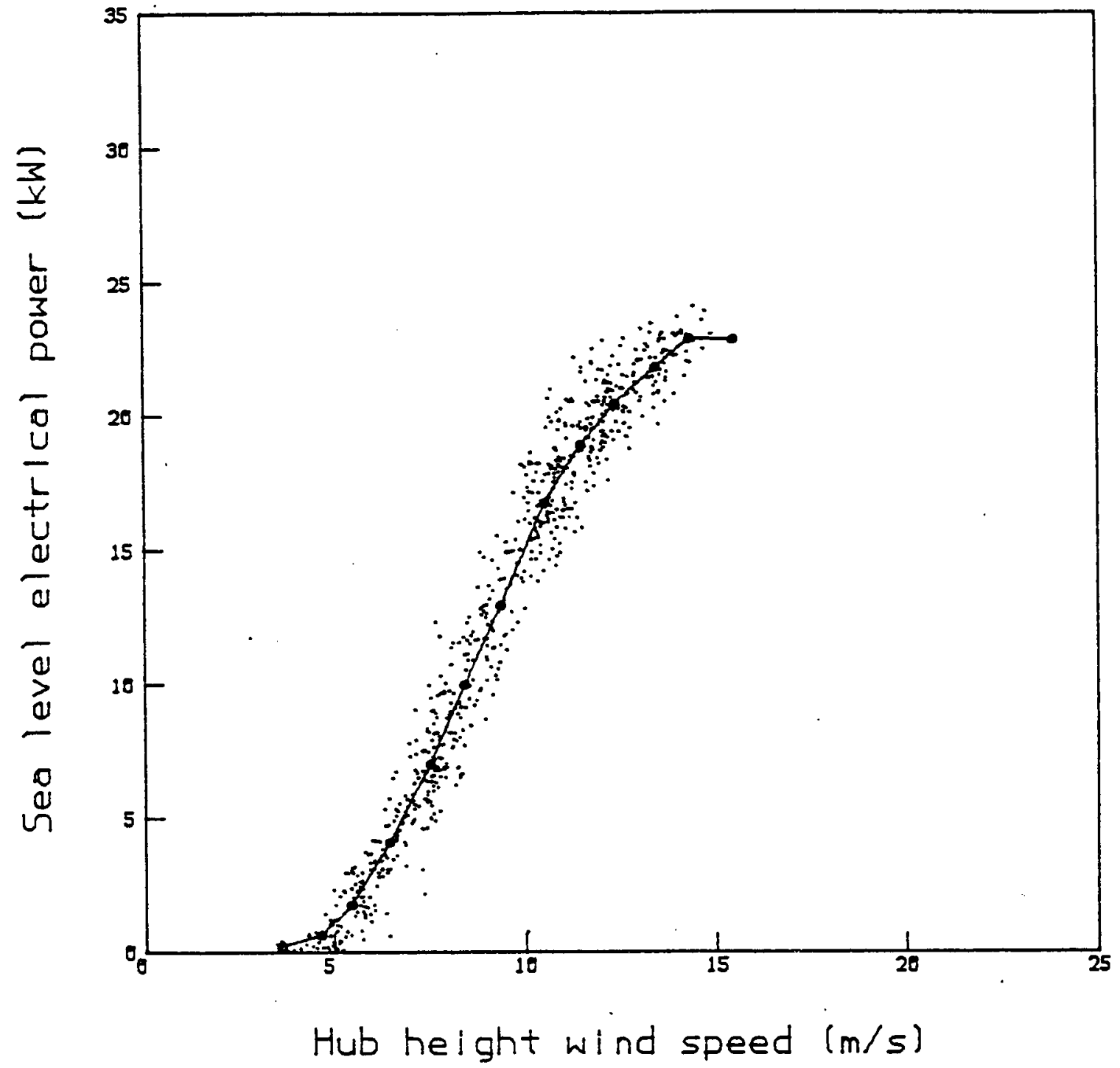

FIGURE 25. Scatter of four-minute average values for rotor with shark's fin tips. 


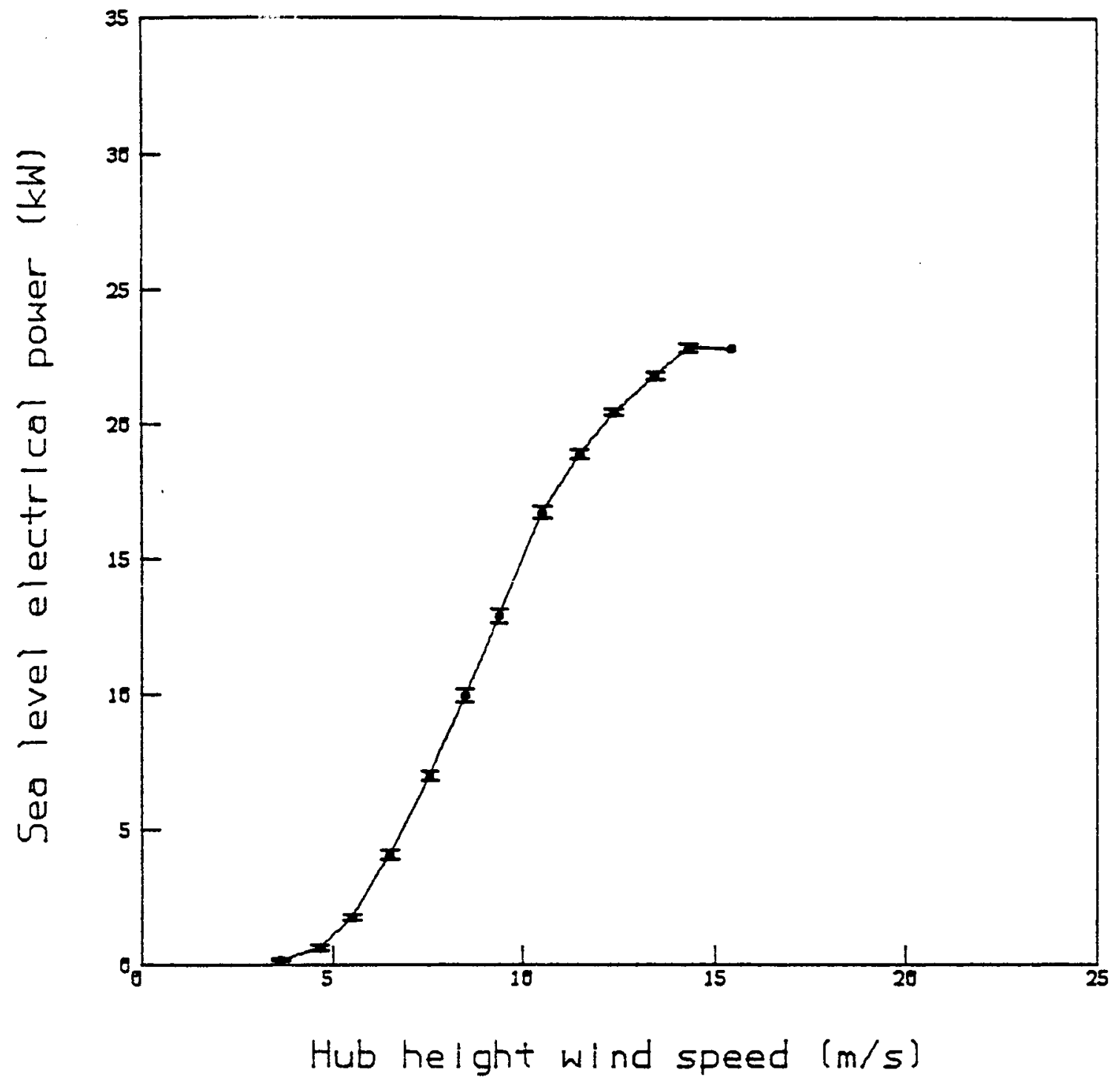

FIGURE 26. Results of bin sort of four-minute averages for rotor with shark's fin tips. 


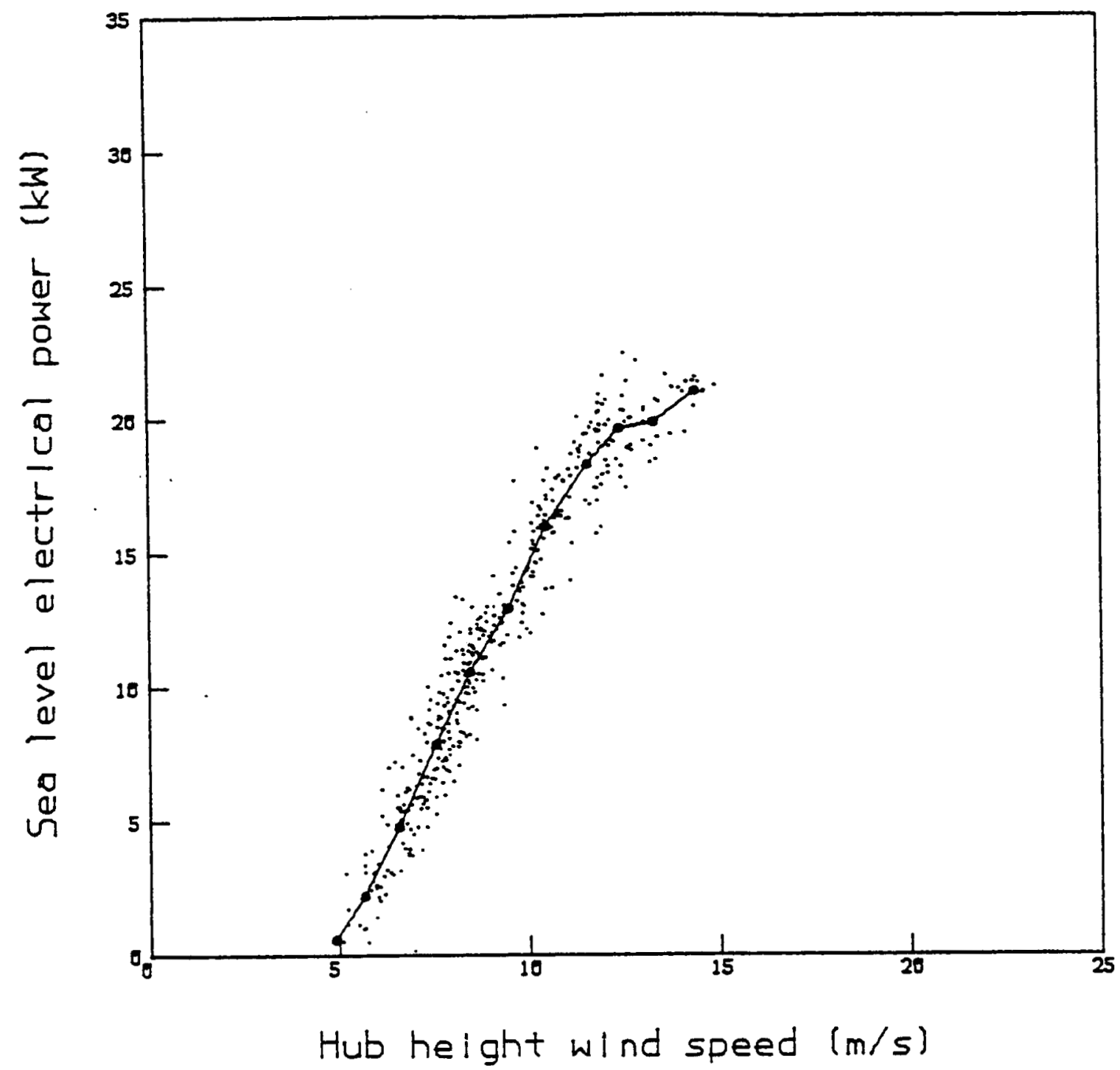

FIGURE 27. Scatter of four-minute average values for rotor with single element tips. 


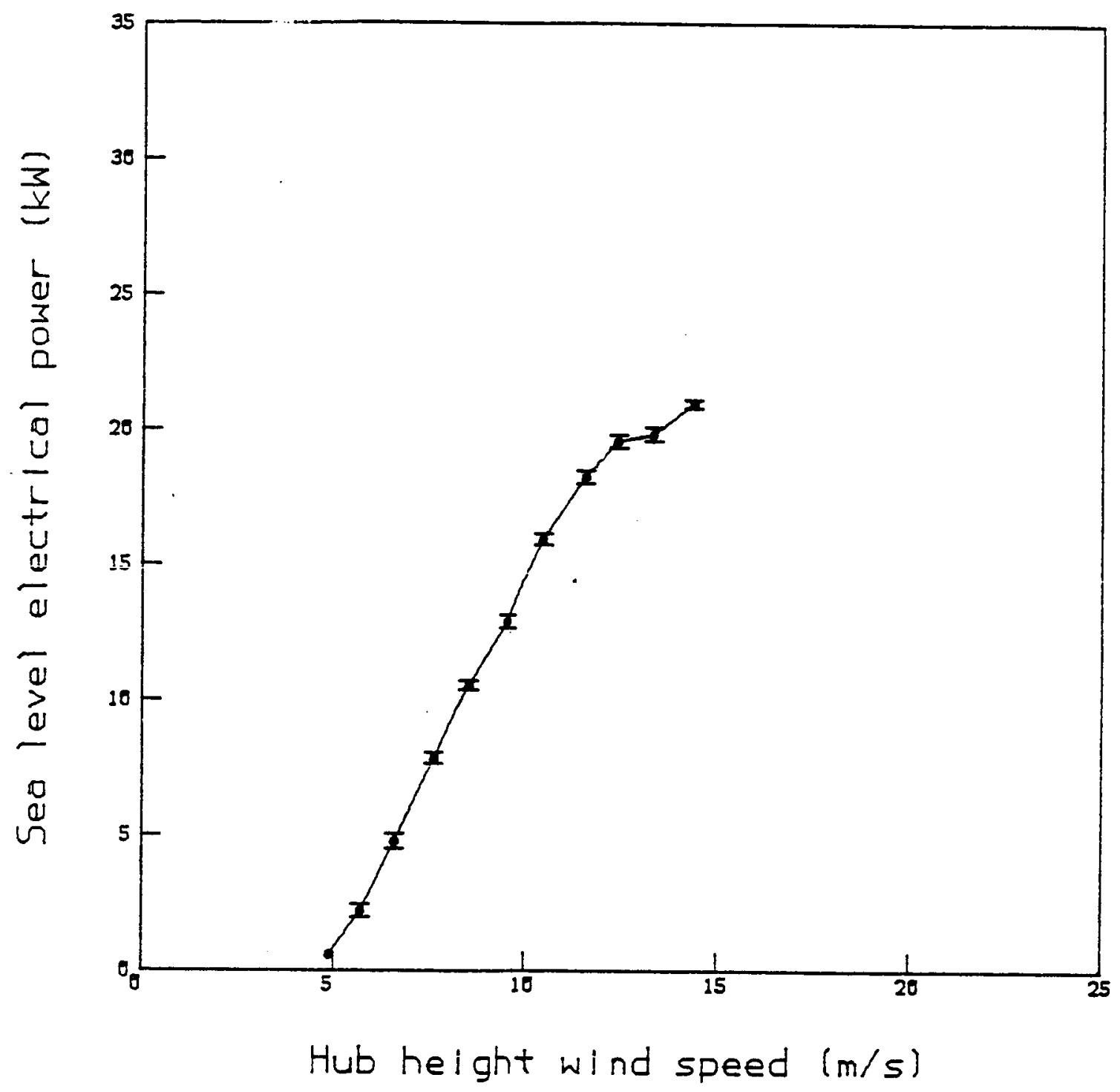
FIGURE 28. Result of bin sort of four-minute averages for rotor with single
element tips. 


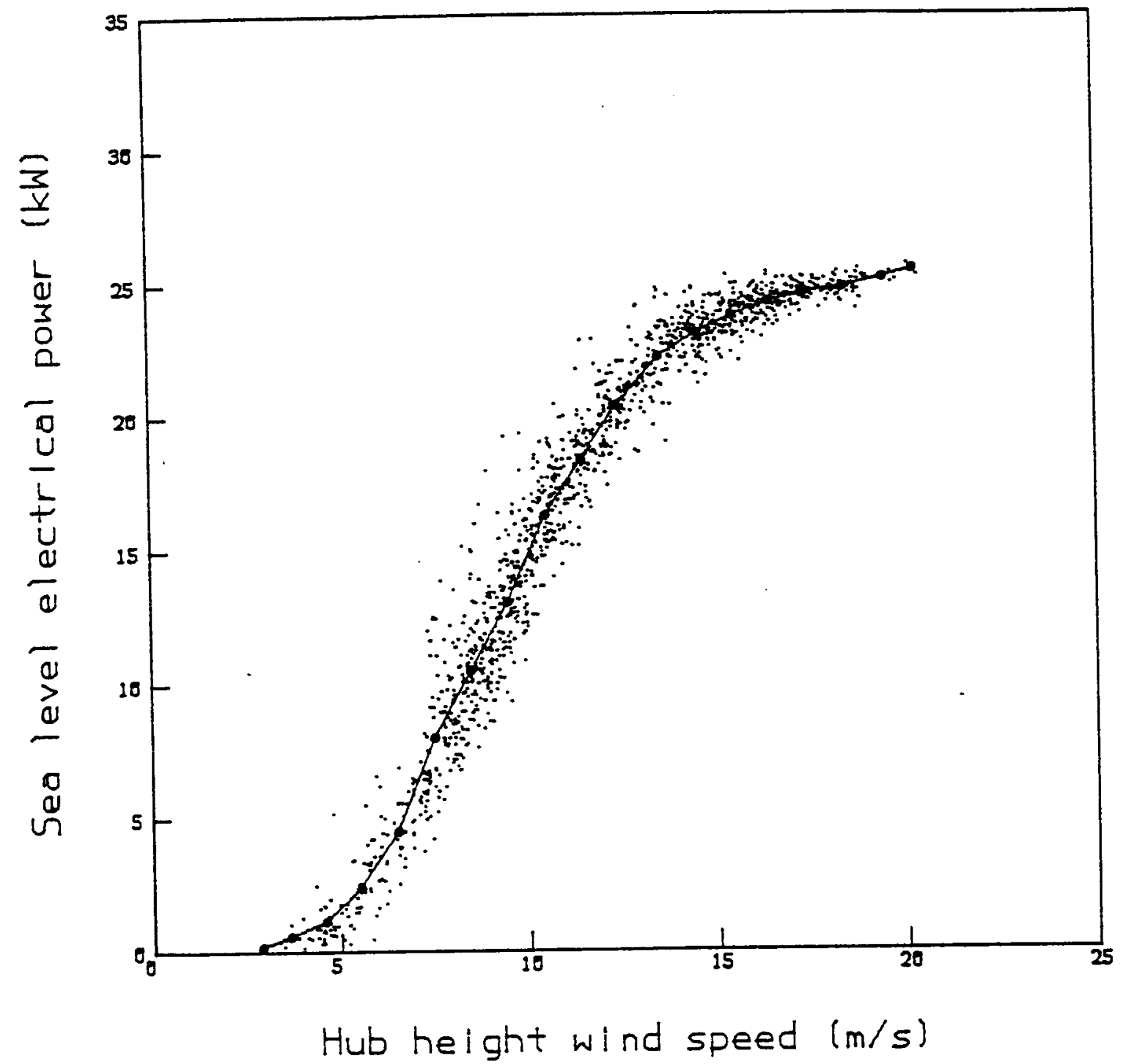

FIGURE 29. Scatter of four-minute average values for rotor with double element tips. 


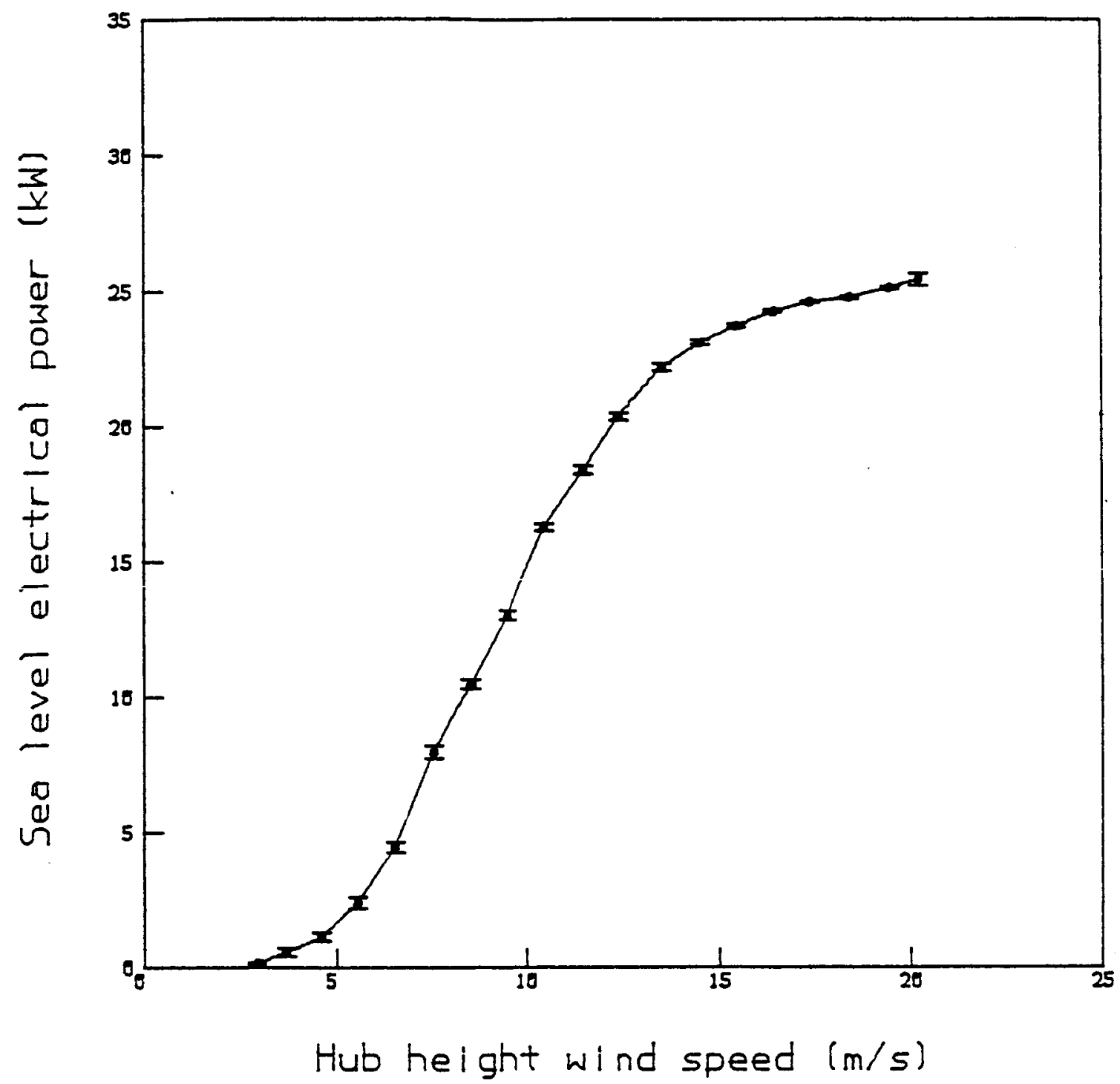

FIGURE 30. Results of bin sort of four-minute averages for rotor with double element tips. 
once every 2.4 seconds. Instantaneous readings of the three wind speed components and electrical power output were recorded at each scan, while pressure and temperature data were recorded only every 10th scan (approximately every 24 seconds) in the interests of data storage economy, and on the assumption that these two parameters change relatively slowly with time.

Figure 31 shows a sample of the raw data as displayed on the field computer after two days of data collection. As expected, there is a considerable amount of scatter due to the physical separation of the turbine and anemometer and because each point represents an instantaneous sample. The values displayed on the figure are not of particular interest here being intended so that the field technician can check for correct operation of the data acquisition system. A small sample of these data, representing about $2.5 \%$ of that shown in Figure 31 , is displayed in Figures 32 and 33 as a function of time; the first showing the individual wind velocity components and the second as wind speed and direction. During this 72 minute period the wind was westerly, the prevailing direction. For the first 11 minutes the wind was too low for power generation but then the turbine came on line and delivered energy to the grid. Although the details of the wind speed and power traces do not appear to correlate well, the general trends do appear to match, for example, the peaks at about 24 minutes and the troughs at about 54 minutes. Clearly some sort of averaging technique was necessary before a bin sort could be performed and the following method was used.

A set of ten consecutive instantaneous wind speed and power values and the corresponding pair of instantaneous pressure and temperature readings was considered one data record. First, sets of ten consecutive records (covering a four-minute period) were formed. In each set each raw data point was then inspected to make sure each channel value looked reasonable; any suspicious point was rejected from the set. The mean wind speed value for the set was then calculated using

$$
\bar{V}=\frac{1}{N} \sum^{N} \sqrt{u_{i}^{2}+v_{i}^{2}}
$$




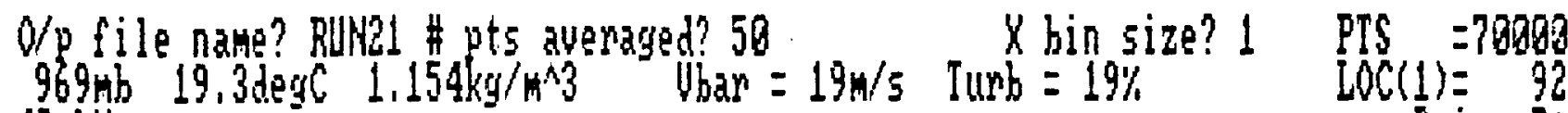
$40 \mathrm{kH}$

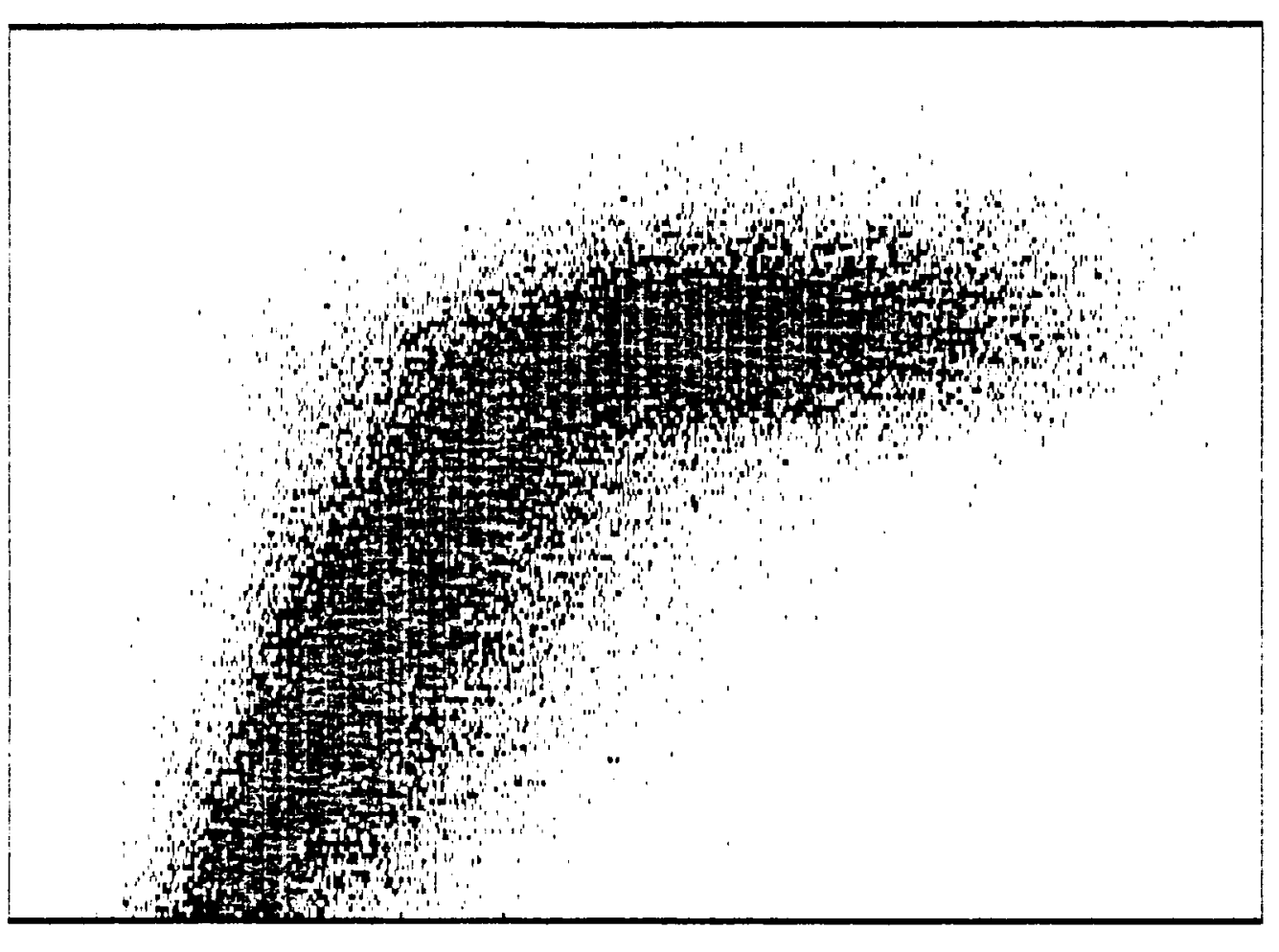

$10-17-198412: 65: 16$

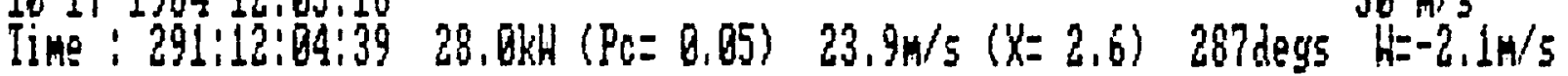

FIGURE 31. Example of two days of raw data as displayed on the field computer. 

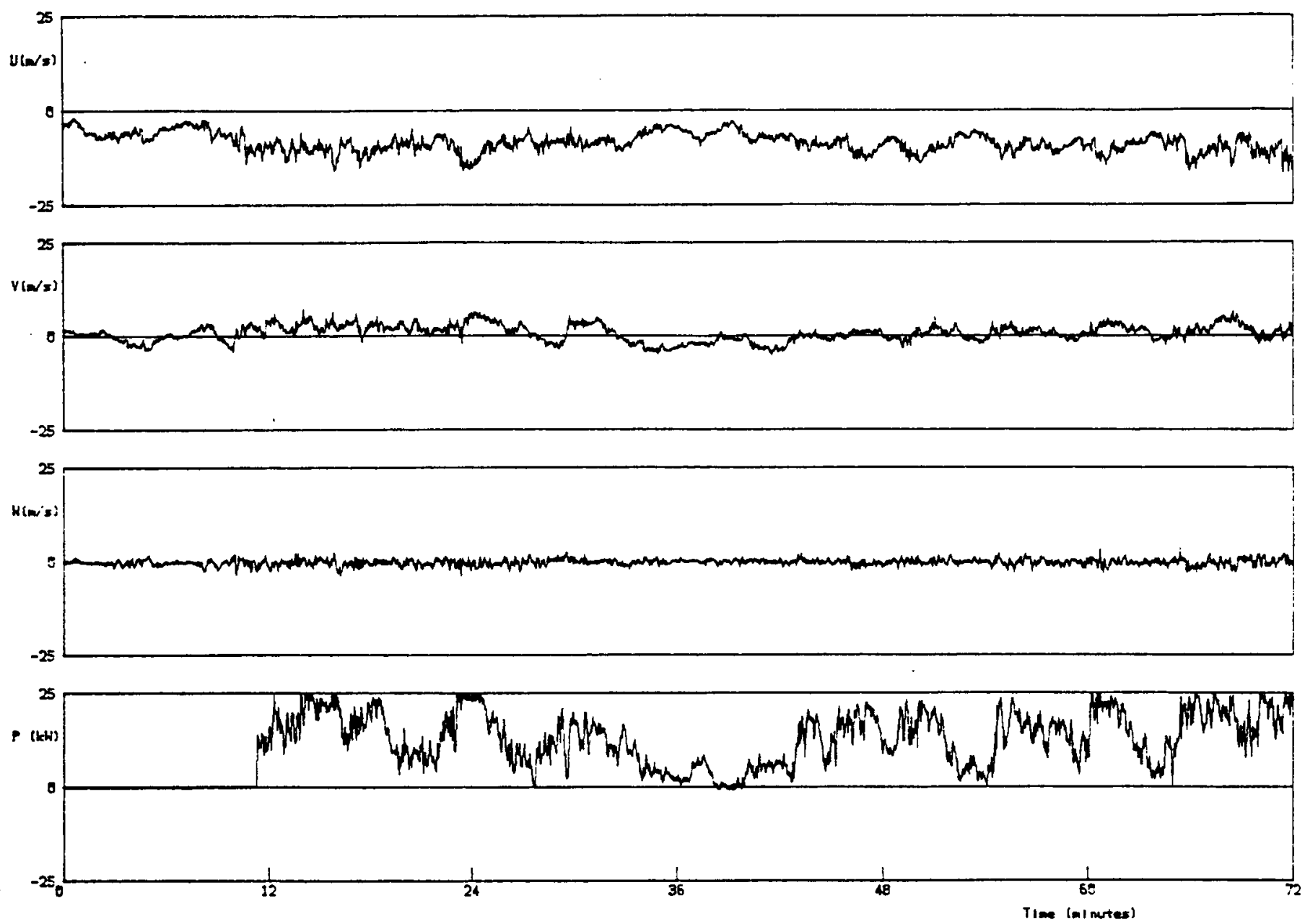

FIGURE 32. Raw data sample from Figure 31 showing wind speed components. 

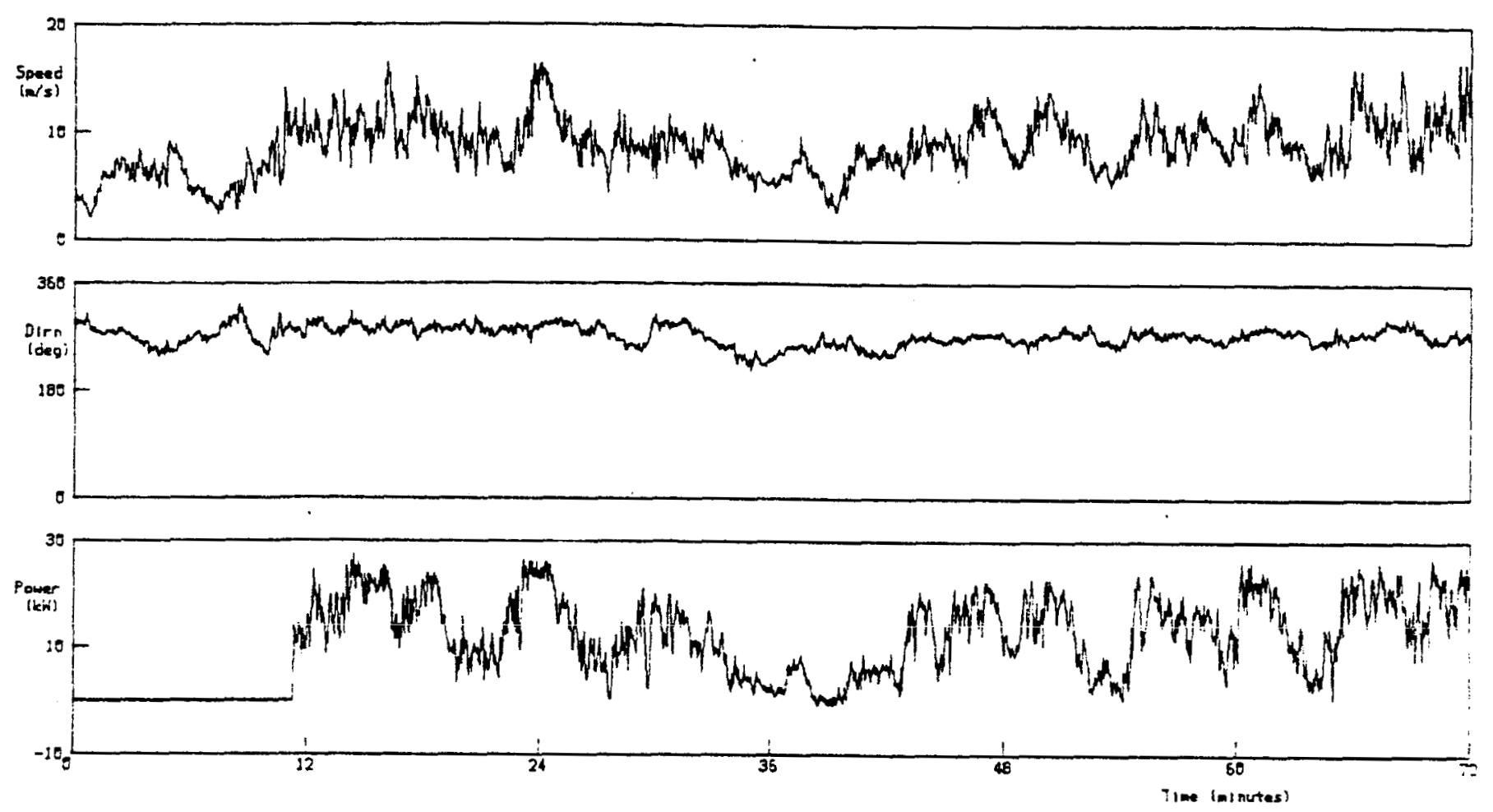

FIGURE 33. Raw data sample from Figure 31 showing wind speed and direction. 
where $\mathrm{N}$ is the number of valid data points and $\mathrm{u}_{\mathrm{i}}$ and $\mathrm{v}_{\mathrm{i}}$ are instantaneous, orthogonal components of wind speed in the horizontal plane. In the field test, the $U$ anemometer was set up to measure easterly wind, the $V$ anemometer northerly and $W$ measured the upwards vertical component. The mean electric power, $\bar{P}$, was then calculated and a simple density correction made to reduce the value to sea level conditions using

$$
\overline{\mathrm{P}}_{\text {sea level }}=\overline{\mathrm{P}} \times \frac{\rho_{\text {sea level }}}{\rho}
$$

It should be noted that this correction is true only if the efficiency of the generator and gearbox is constant. Although this is not so in practice, it is an acceptable approximation for the purposes of these tests. The actual value of efficiency, if constant, does not change the magnitude of the correction.

The pair of values $\bar{V}$ and $\bar{P}_{\text {sea level }}$ together form a single reduced data point, which appears in the figures as a dot, representing a four-minute average of conditions.

The next stage was to perform a bin sort on the four-minute averages. Before this however, any reduced point for which the wind direction was within $\pm 45^{\circ}$ of north was rejected to avoid including wake interference effects from upwind turbines. In addition, any reduced point with an average power output of less than 50W was rejected to avoid biasing the bin sort by data collected during times when the turbine was being held off-line by, for example, an out-of-balance trip. Although this causes some distortion of the performance curve at the low wind speeds around cut in, this part of the curve is not of primary interest in these tests.

The curve shown on each of the figures is the result of a bin sort using $1 \mathrm{~m} / \mathrm{s}$ wide bins. The error bars shown for each bin average represent the magnitude of one standard deviation (or error) of the mean of the power data. The standard error is a statistical measure of the accuracy of a sample mean as an estimator of the "true" population mean (i.e., that obtained from a very large sample). For 
example, in these figures, each bin holds between 0 and about 100 points, for which we can determine a set of bin averages of wind speed and power output. If the test. was repeated however, we would typically not expect to find exactly the same set of bin average values; if our field test was repeated a large number of times, it would be found that the frequency distribution of the bin average for each bin was Gaussian in shape and centered on the "true" bin average. The standard deviation of this Gaussian is what is termed the standard error of the sample mean. In other words, $68 \%$ of the time the "true" mean power level for a given bin lies with in the error bar limits shown on the figures. The standard error of a sample mean, $\sigma_{e}$, can be calculated as the standard deviation of the population, $\sigma$, divided by the square root of the number of points in the sample, $n ;$ i.e., $\sigma_{e}=\frac{\sigma}{n}$. Since we cannot know the standard deviation of the whole population, the standard deviation of the sample, $\mathrm{S}$, was used in place of $\sigma$ in calculating $\sigma_{\mathrm{e}}$ where

$$
s^{2}=\frac{\sum\left(P_{i}-\bar{P}\right)^{2}}{n-1}
$$

where $P_{i}$ represents the set of four minute average values of power in the bin and $\bar{P}$ is the mean value of $P_{i}$.

Since $n$ was fairly large $(\sim 100)$ in these tests, we can expect the calculated value of $\sigma_{e}$ to be a reasonably good approximation of the correct value. It is noted that the error bars shown represent the error of the mean, not the probable deviation of any given point. This latter deviation is approximately ten times as large and is observed as the "cloud" of data points enveloping the mean line.

It can be seen that the data scatter of the reduced data is quite small, on the order of $\pm 2 \mathrm{~kW}$, and that a distinct band of data exists containing the power curve. The error bars of the mean are much smaller, on the order of $\pm 0.2 \mathrm{~kW}$, which gives a high degree of confidence in the accuracy of the resulting mean power curve. A secondary indicator which gives us confidence in the accuracy of the results is the fact that the resulting power curves are smooth -- a characteristic expected of a real machine. Figure 34 shows the effect of the data reduction method on the two 


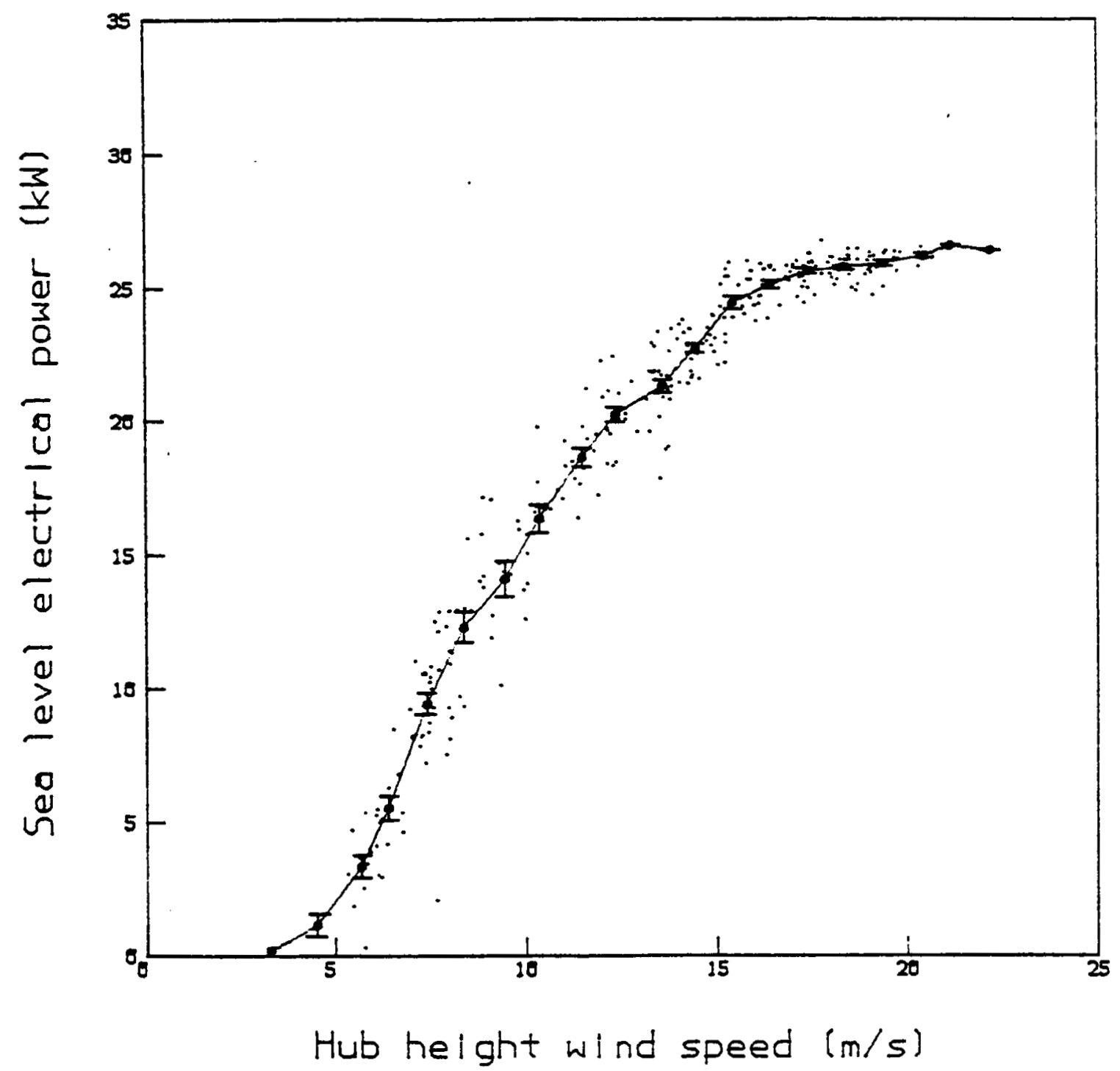

FIGURE 34. Result of applying the data reduction method to the raw data shown. in Figure 31 . 
days of raw data shown in Figure 31. The graph shows that even these 70,000 data points are insufficient to produce a smooth power curve.

The tests on the single-element tips were cut short when the large male attachment pin pulled out of one of the tips and the tip was thrown and destroyed. The test turbine was undamaged by the loss of the tip, being shut down by its vibration sensor. Following this incident it was decided to strengthen the double-element tips prior to their installation and testing.

\section{DISCUSSION OF RESULTS}

In order to validate the data acquisition system, data were collected for the baseline tips and compared with the manufacturer's data. The results of this comparison is shown in Figure 35. The measurements agree quite well with Carter's data except at high power levels.

AV'S PROP model was then run for the true Carter blade shape defined in Figures 5 and 6. The airfoil performance. data for the NACA 23012 shown in Figures 7 and 8 were used for the outer $50 \%$ of the blade, while data from the same source (Miley, 1982) for the NACA 23015 at a Reynolds number of 700,000 were used for the inboard half. The Prandtl tip loss model was used with a $4^{\circ}$ cone angle and swirl effects included. The rotor rpm was set at 123 to represent the slip in the induction generator. Drive train losses were modeled using:

\section{Electrical Power $=92.5 \% \times$ Shaft Power $-7.5 \% \times$ Rated Power}

to represent both a fixeci bearing loss at constant rpm and a generator loss proportional to power. It can be seen that the performance predictions of the PROP model are within 10\% of the measured performance over the whole range of wind speeds encountered in the test and within a few percent over most of that range. Certainly PROP's predictions match the measured data far better than they do the manufacturer's specifications. The main region of difference is in the stalled region where PROP's predictions are slightly high. It should also be noted that PROP tends to slightly underpredict performance in the stalled region which 


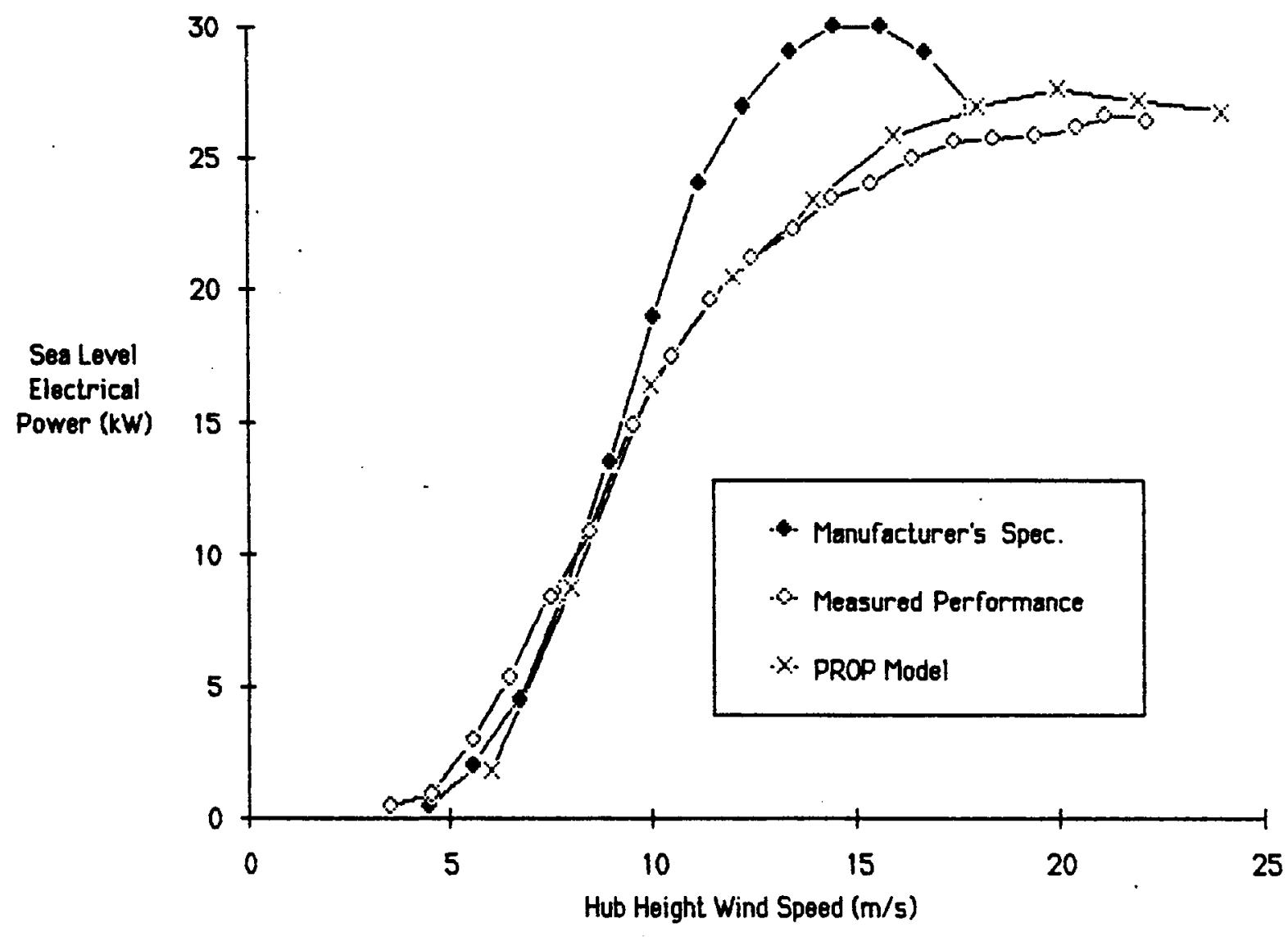

FIGURE 35. Assessment of measured performance of rotor with baseline tips. 
makes this difference slightly worse. It is believed that this difference is at least partly due to dirt and bugs on the leading edge of the blades. As part of the routine maintenance operations at the windfarm test site, each machine is lowered to have its blades washed approximately every two weeks in order to maximize performance. Figure 36 shows the quantity of dirt and bugs that was accumulating in this time interval. In viewing the photo, note that the black area near the tip junction is spray paint, the bug/dirt roughness referred to is along the leading edge of the rotor on the main blade and tip. Miley (1982) shows at substantial performance degradation of the 23012 when the surface is roughened and it is reasonable to expect that this leading edge dirt resulted in both a lower maximum $C_{L}$ and higher $C_{D}$ sufficient to account for the observed difference between PROP's predictions and measurements. It is thought that the reason for the manufcturer's curve being so much higher is because that data were collected with the blades set at a higher pitch setting. The pitch setting on the test turbine was checked periodically during the field test but each time is was found to be correct.

As a further check on the vaiidity of the results, performance data for the blades with the baseline tips were collected both before and after the tests on the new tip devices to ensure that the performance of the reference rotor did not change during the experiment. The data obtained are shown in Figures 37 and 38 and compared in Figure 39. Although the distribution of wind speeds during the pre- and post-test reference checks was not the same, the overall performance of the system was almost identical as can be seen in Figure 39, the largest deviation being around $8 \mathrm{~m} / \mathrm{s}$ where data were sparse and the pre-test data show an unlikely kink.

The performance of the four tip configurations are compared in Figure 40. It can be seen that none of the new tip devices offers an improvement over the simple baseline shape, in fact, their performance is slightly poorer by about $1 \mathrm{~kW}$ over the measured range of wind speeds. Since there is little overlapping of the error bars, the measured differences are considered significant.

Since the difference between the performance of each of the new tips and the baseline tips is fairly constant and does not vary with wind speed, it is 


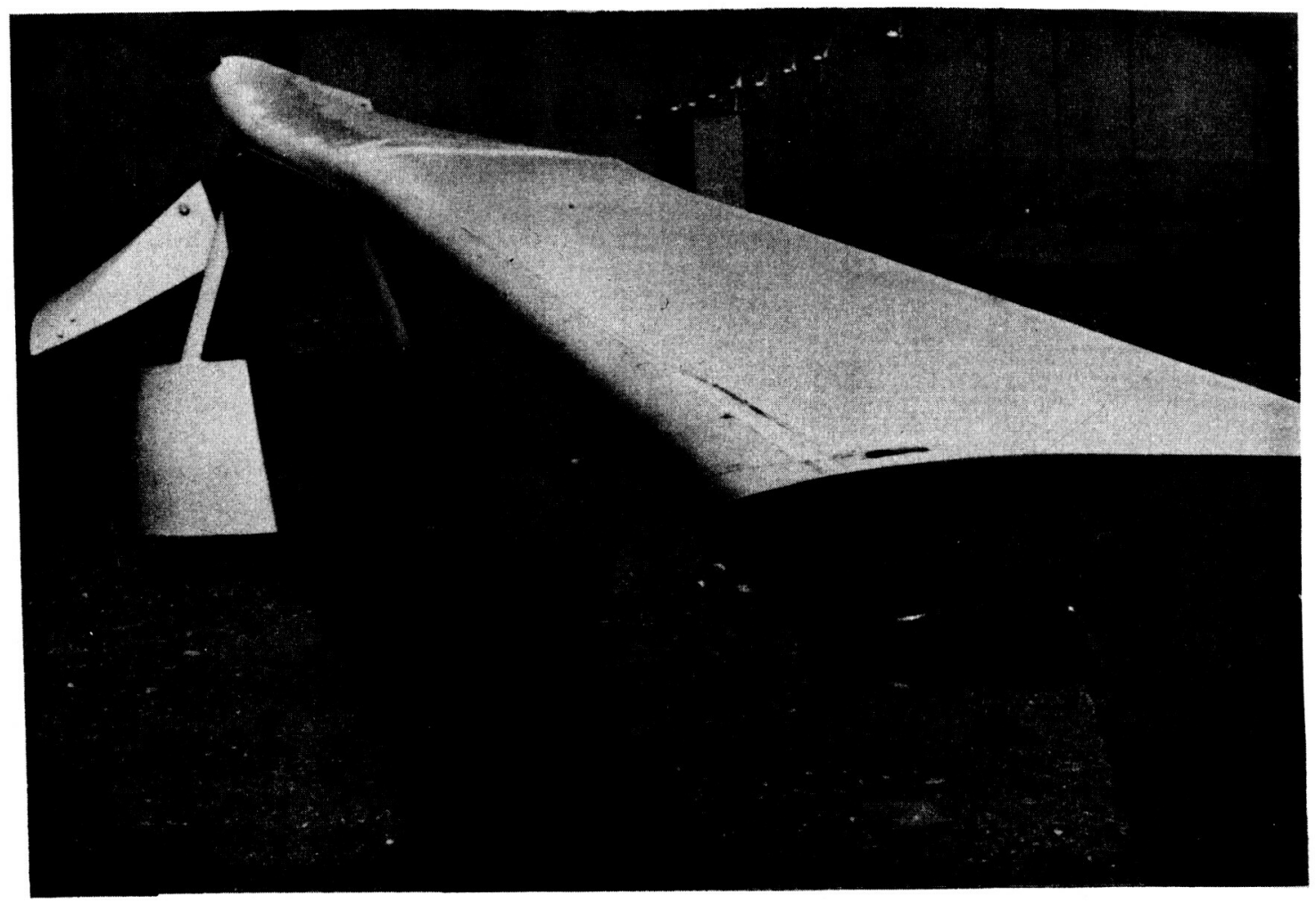

FIGURE 36. Accumulation of dirt and bugs on leading edge of blades. Note the increased accumulation on the outer part of the blade.

ORIGINAL PAGE IS

OF POOR QUALTY 


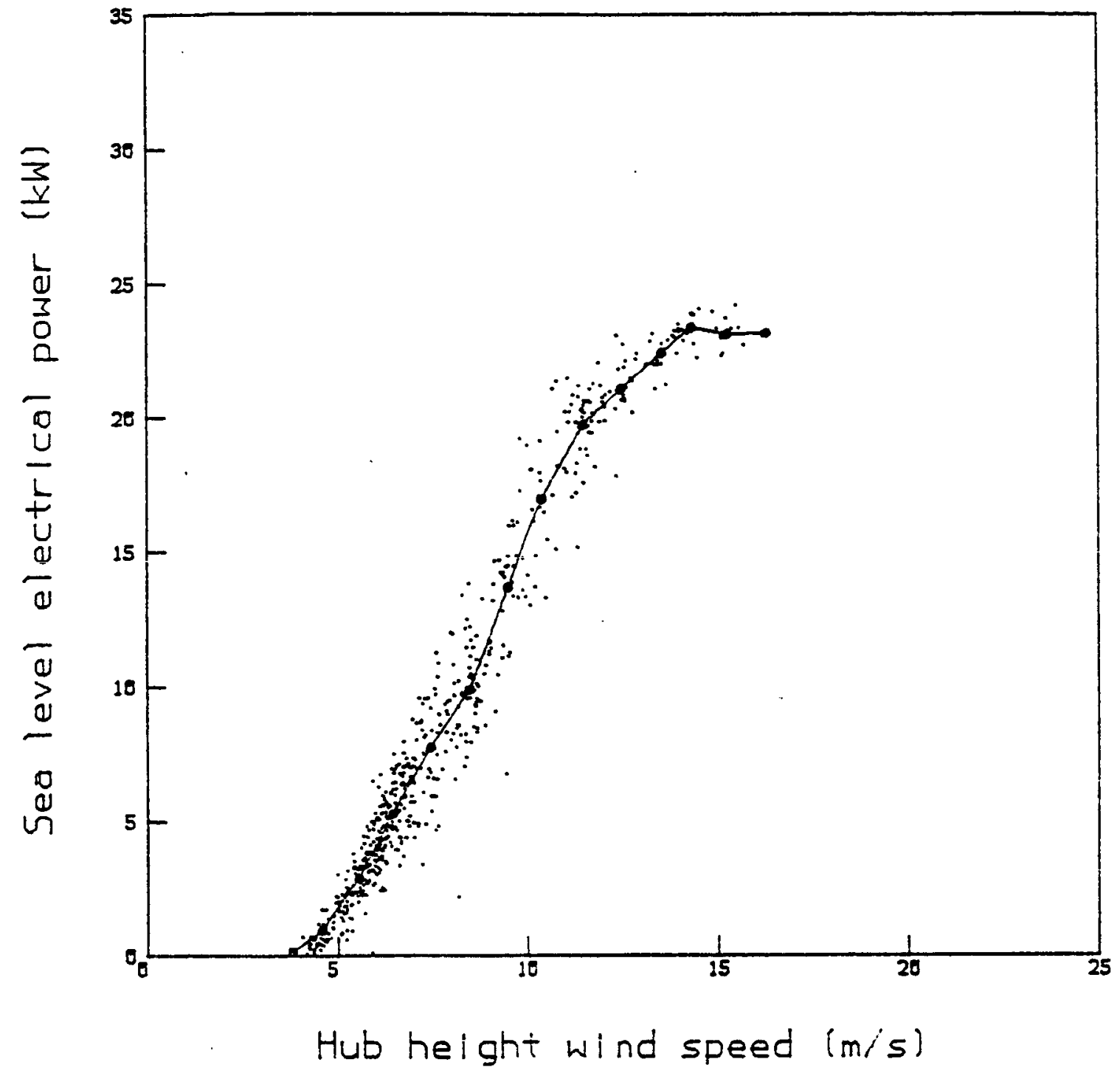

FIGURE 37. Measured performance of rotor with baseline tips before tip device tests. 


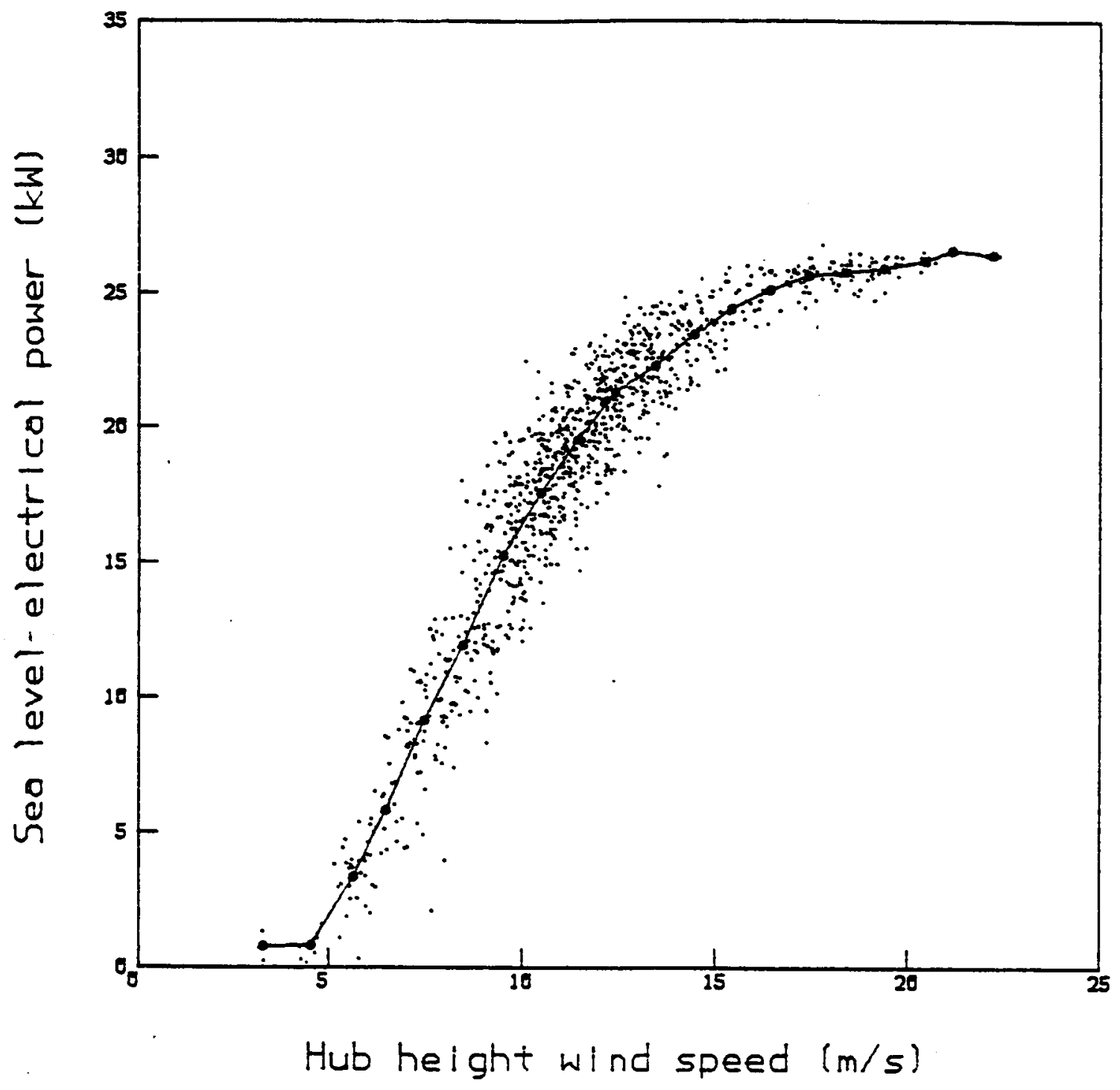

FIGURE 38. Measured performance of rotor with baseline tips after tip device tests. 


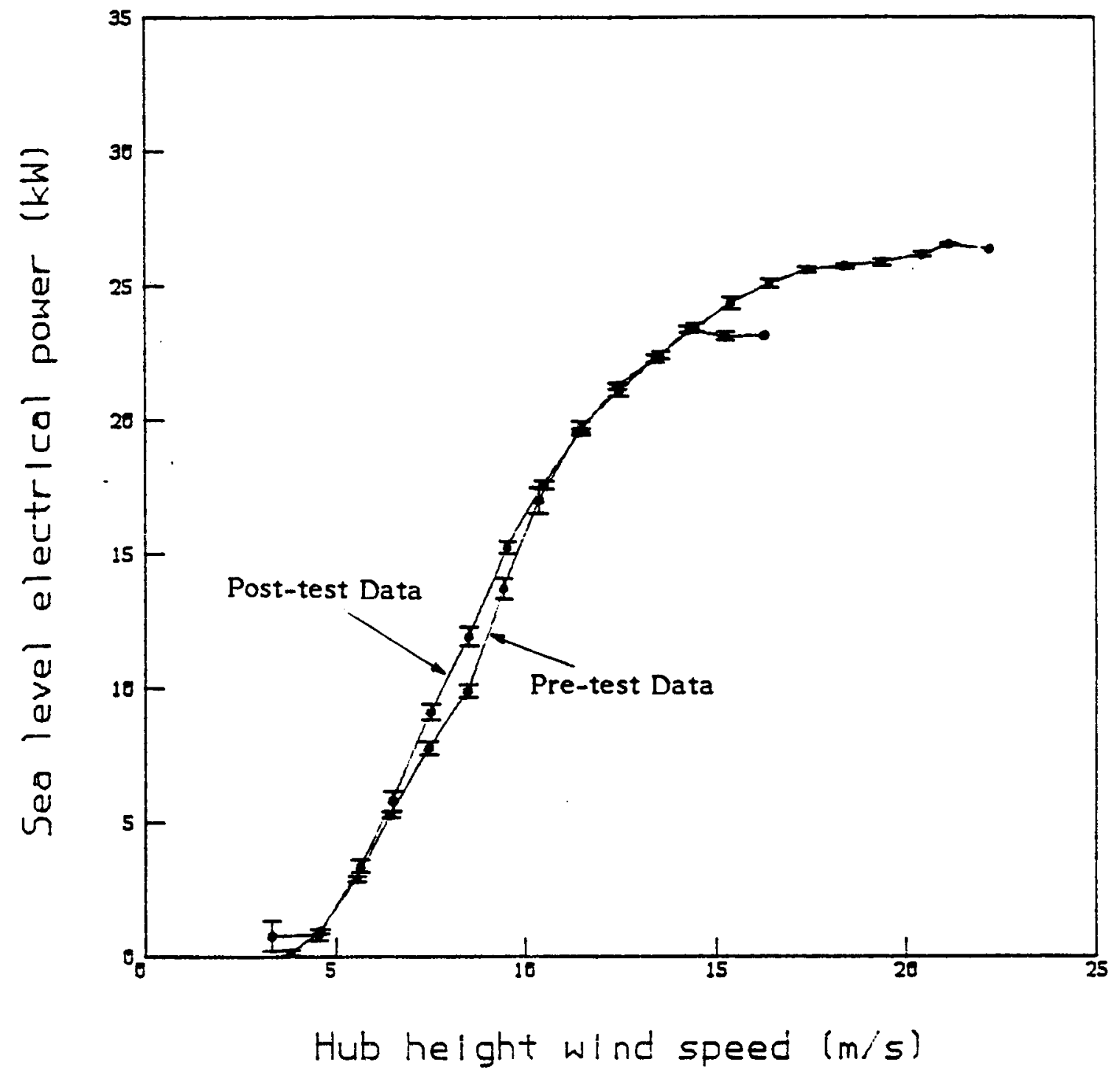

FIGURE 39. Comparison of pre- and post-test performance data for rotor with baseline tips. 


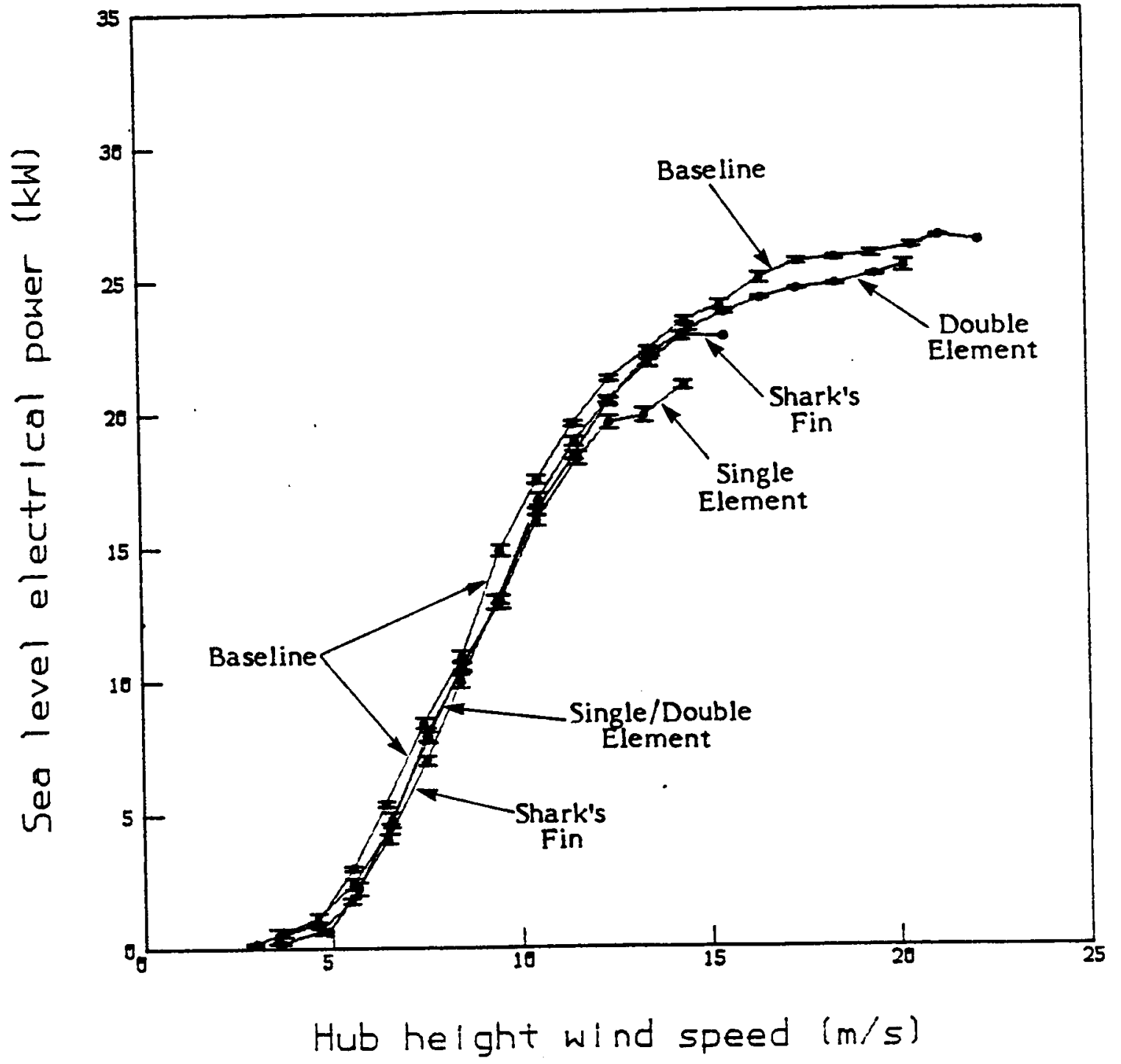

FIGURE 40. Comparison of performance of tip devices. 
suspected that the reduced performance is due either to excessive drag on the tips or because the tips simply were not generating the expected lift. If the tip elements were stalling part way up the power curve, we would expect to see this by an increasing deviation from the baseline curve as the wind speed increased. The single element device may have been entering such a region at about $12 \mathrm{~m} / \mathrm{s}$ but the data there are too sparse for a firm conclusion to be drawn.

During the field test it was noticed that both the single and double-element devices were significantly more noisy than the baseline tips implying that there were some excessive drag sources causing turbulence.

If more progress is to be made, it appears that either a better computer model is required or a more extensive field program is necessary either testing a large number of different tips or, better, testing variable geometry tips adjustable either by lowering the turbine and manually resulting angles or replacing tip elements or fairings or else remotely using radio control. Flow visualization for the rotating blades would also be an extrernely useful diagnostic aid but would be difficult to implement. One way to achieve this would be to mount a camera with automatic exposure control and a motor drive at the hub center with a long focal length lens pre-focused on tufts at the tips. Radio control could be used to operate the shutter at various wind speeds. This method would, however, still leave portions of the tip out of view.

The promising results obtained $c n$ nonrotating wings make it difficult to accept that tip devices could not improve wind turbine performance.

\section{CONCLUSIONS AND RECOMMENDATIONS}

The following conclusion. can be drewn:

1) None of the three tip shapes tested exhibited any meaningful performance improvements. All had marginal (but statistically significant) performance degradations compared with the baseline rotor. 
2) Although tip devices have been successful on nonrotating wings these tests suggest that tip devices may not improve rotor performance. However, it should be remembered that the tip shapes tested represent a minute sampling of a very wide range of shapes and settings and successful designs may exist.

3) The Wilson/Lissaman PROP model was shown to be in excellent agreement with tests for the baseline rotor.

4) The analytical techniques used, believed to be the best available, are unable to predict the performance effects of tip modifications even qualitatively and, therefore, should not be used. The discrepancies are possibly due to a) too crude a vortex lattice, b) improper wake geometry modeling, and c) inadequate treatment of viscous effects.

5) The turbine performance data collection system developed in this project worked very well and testing over quite small time periods provided sufficient data for good statistical results. It is a valuable tool for remote field tests and it provides a real-time "ground truth" test of true rotor field performance.

6) The fixed geometry of the tested tip shapes made it impossible to "tune" or adjust the rotor by making small angular changes, as is normally done in lifting surface testing. The difficulty of implementing flow visualization methods compounded the "tuning" difficulties.

The following recommendations are made:

1) The turbine performance data collection system should be exploited for other rotor improvement devices such as vortex generators and novel airfoil sections.

2) An improved computer model is clearly required, to ameliorate the deficiences noted in conclusion 2). However at the current state of the 
art, the most cost-effective development method is probably field or wind tunnel test of actual rotors rather than computer modeling.

\section{REFERENCES}

Braden, J.A., C.G. Phillips, and D.E. Lilley (1981 and 1982): Wind tunnel study of conical chamber wing tips. Lockheed-Georgia Company report Nos. LG81WP7274-101 and LG82WP7274-105.

Flechner, S.G., P.F. Jacobs, and R.T. Whitcomb (1976): A high subsonic speed wind-tunnel investigation of winglets on a representative second generation jet transport wing. NASA TN D-8264, July.

Gifford, R.V., and C.P. van Donn (1982): The integration of wing tip devices for light general aviation aircraft. ICAS-82-1.4.4.

Gyatt, G.W., A.D. Zalay, and B. Hibbs (1982): Optimization of the dynamic inducer wind energy system. AeroVironment Inc. Report AV-FR-83/547 (to be issued as SERI/DOE report in 1985).

Hackett, J.E. (1980): Vortex drag reduction by aft mounted diffusing vanes. ICAS-80-13.4, October.

Hackett, J.E., and C.G. Phillips (1980): Low speed tests on various wing tip devices for drag reduction. Lockheed-Georgia Company report No. LG80ER0049.

Hackett, J.E. (1981): Vortex drag reduction by diffusing vanes: Design for the "Thrush" agricultural aircraft. SAE Technical Paper 810605.

Hoerner, S.F. (1965): Fluid dynamic drag. Published by the author, Brick Town, New Jersey.

Ishimitsu, K.K. (1976): Aerodynamic design and analysis of winglets. Presented at AIAA Aircraft Systems and Technology Meeting, Dallas, TX, September.

Miley, S.J. (1982): A catalog of low Reynolds number airfoil data for wind turbine applications. Prepared for Rocky Flats Plant Wind Systems Program, Golden, CO, under subcontract No. PFY12781-W, February.

Spillman, J.J. (1978): The use of wing tip sails to reduce vortex drag. Aeronautical J., Paper No. 618, 387-395, September.

Sullivan, J.P., L.K. Chang, and C.J. Miller (1982): Effects of proplets and bi-blades on the performance and noise of propellers. School of Aeronautical and Astronautical Engineering, Aerospace Sciences Laboratory, Purdue University, Report ASL 82-415, March. 
Whitcomb, R.T. (1976): A design approach and selected wind tunnel results at high subsonic speeds for wing-tip mounted winglets. NASA TN D-8260, July.

Wilson, R.E., and P.B.S. Lissaman (1974): Applied aerodynamics of wind power machines. Supported by NSF RANN uñder Grant No. GI-41840, May. 


\section{APPENDIX A \\ Specification Summary of Test Turbine \\ Carter Wind Systems Model 25}

\section{General:}

Horizontal axis

Downwind rotor

Free yaw

32 feet diameter

2 blades

$80 \mathrm{ft}$ hub height

$120 \mathrm{rpm}$ rotor

$25 \mathrm{~kW}$ rated power

Cut in around $4 \mathrm{~m} / \mathrm{s}$ (rpm controlled)

No cut out wind speed

3 phase induction generator, $1800 \mathrm{rpm}, 480 \mathrm{~V}$

\section{Blades:}

Chord, see Figure 5

Twist, see Figure 6

Airfoil: 23012 at tip, 23021 at root

$4^{\circ}$ precone

Material: fiberglass and PVC foam

\section{Control System:}

Stall controlled rotor, fixed pitch

Disk brake operated manually or by vibration sensor

Overspeed control by blade pitching due to excess centrifugal loads

On-line/off-line control by rpm sensing and SCR switching 
Catalog of Tip Device Designs

and Their Predicted Performance

Single element tip geometries

$\begin{array}{ccccccccc}\text { TIP } & \begin{array}{c}\text { ROOT } \\ \text { POSITION } \\ \%\end{array} & \text { SPAN } & \begin{array}{c}\text { ROOT } \\ \text { CHORD }\end{array} & \begin{array}{c}\text { TIP } \\ \text { CHORU }\end{array} & \begin{array}{c}\text { ROOT } \\ \text { INCIDENCE } \\ \text { deg }\end{array} & \begin{array}{c}\text { TIP } \\ \text { INCIDENCE } \\ \text { deg }\end{array} & \begin{array}{c}\text { SWEEP } \\ \text { deg }\end{array} & \begin{array}{l}\text { CANT } \\ \text { deg }\end{array} \\ 1 & 25 & .05 & .0162 & .0162 & 0 & 0 & 0 & 4 \\ 2 & 25 & .05 & .0324 & .0324 & 0 & 0 & 0 & 4 \\ 3 & 25 & .05 & .0488 & .0488 & 0 & 0 & 0 & 4 \\ 4 & 25 & .1 & .0325 & .0325 & 0 & 0 & 0 & 4 \\ 5 & 25 & .05 & .0432 & .0216 & 0 & 0 & 0 & 4 \\ 6 & - & - & - & - & - & - & - & - \\ 7 & 25 & .05 & .0082 & .0082 & 0 & 0 & 0 & 4 \\ 8 & 25 & .05 & .0432 & .0216 & 1.1 & 1.1 & 0 & 26.5 \\ 9 & 25 & .05 & .0432 & .0216 & 2.7 & 2.7 & 0 & 49 \\ 10 & 25 & .05 & .0424 & .0216 & 2.77 & 2.77 & 0 & 71.5 \\ 11 & 25 & .05 & .0432 & .0216 & 3 & 3 & 0 & 94 \\ 12 & 25 & .05 & .0432 & .0216 & 2.77 & 2.77 & 0 & 116.5 \\ 13 & 25 & .05 & .0432 & .0216 & 2.1 & 2.1 & 0 & 139.0 \\ 14 & 25 & .05 & .0432 & .0216 & 1.1 & 1.1 & 0 & 161.5 \\ 15 & 25 & .05 & .0432 & .0216 & 0 & 0 & 0 & 184 \\ 16 & 25 & .05 & .0162 & .0162 & -2 & -2 & 0 & 4 \\ 17 & 25 & .05 & .0162 & .0162 & -4 & -4 & 25 & 4 \\ 18 & 25 & .1 & .0162 & .0162 & 0 & 0 & 0 & 4 \\ 19 & 25 & .1 & .0081 & .0081 & 0 & 0 & 0 & 4 \\ 20 & 25 & .05 & .0162 & .0162 & -4 & -4 & 0 & 4 \\ 21 & 25 & .05 & .0162 & .0162 & -6 & -6 & 0 & 4 \\ 22 & 25 & .05 & .0162 & .0162 & 0 & 0 & 25 & 4 \\ 23 & 25 & .05 & .0162 & .0162 & -4 & -4 & 35 & 4 \\ 24 & 25 & .05 & .0162 & .0162 & -6 & -6 & 25 & 4 \\ 25 & 50 & .05 & .0162 & .0162 & 0 & 0 & 0 & 4 \\ 26 & 50 & .05 & .0162 & .0162 & -2 & -2 & 0 & 4 \\ 27 & 50 & .05 & .0162 & .0162 & -4 & -4 & 0 & 4 \\ 28 & 50 & .05 & .0162 & .0162 & 0 & 0 & 25 & 4 \\ 29 & 75 & .05 & .0162 & .0162 & 0 & 0 & 0 & 4 \\ 30 & 75 & .05 & .0162 & .0162 & -2 & -2 & 0 & 4\end{array}$


Single element tip geometries

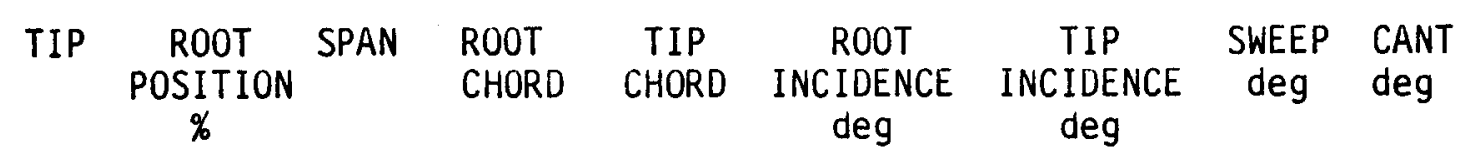

$\begin{array}{rllllllll}31 & 75 & .05 & .0162 & .0162 & -4 & -4 & 0 & 4 \\ 32 & 75 & .05 & .0162 & .0162 & 0 & 0 & 25 & 4 \\ 33 & 50 & .06 & .0162 & .0162 & -4 & -4 & 0 & 4 \\ 34 & 50 & .05 & .017 & .017 & -4 & -4 & 0 & 4 \\ 35 & 50 & .06 & .017 & .017 & -4 & -4 & 0 & 4 \\ 36 & 50 & .05 & .0162 & .0162 & -6 & -6 & 0 & 4 \\ 37 & 50 & .05 & .0162 & .0162 & -2 & -6 & 0 & 4 \\ 38 & 50 & .05 & .0216 & .0108 & -4 & -4 & 0 & 4 \\ \star 39 & 50 & .05 & .0216 & .0108 & -2 & -6 & 0 & 4 \\ 40 & 50 & .05 & .0162 & .0162 & -4 & -4 & 10 & 4 \\ 41 & 50 & .05 & .0162 & .0162 & -4 & -4 & -10 & 4 \\ 42 & 50 & .05 & .0162 & .0162 & -4 & -4 & 0 & 8 \\ 43 & 50 & .05 & .0162 & .0162 & -4 & -4 & 0 & 0 \\ 44 & 25 & .0325 & .065 & .01 & 0 & 0 & 56 & 0 \\ 45 & 25 & .0325 & .065 & .01 & 0 & 0 & 56 & 180\end{array}$

*Selected tip 
"Shark fins" type tip geometries

$\begin{array}{ccccccccc}\text { TIP } & \begin{array}{c}\text { ROOT } \\ \text { POSITION } \\ \%\end{array} & \text { SPAN } & \begin{array}{l}\text { ROOT } \\ \text { CHORD }\end{array} & \begin{array}{c}\text { TIP } \\ \text { CHORD }\end{array} & \begin{array}{c}\text { ROOT } \\ \text { INCIDENCE } \\ \text { deg }\end{array} & \begin{array}{c}\text { TIP } \\ \text { INCIDENCE } \\ \text { deg }\end{array} & \begin{array}{c}\text { SWEEP } \\ \text { deg }\end{array} & \begin{array}{c}\text { CANT } \\ \text { deg }\end{array} \\ 1000 & 25 & .065 & .065 & .065 & 3 & 3 & 0 & 90 \\ 1001 & 25 & .065 & .065 & .065 & 3 & 3 & 45 & 90 \\ 1002 & 25 & .065 & .065 & .022 & 3 & 3 & 0 & 90 \\ 1003 & 25 & .065 & .065 & .022 & 3 & 3 & 45 & 90 \\ 1004 & 25 & .065 & .065 & .022 & 3 & 3 & 30 & 90 \\ 1005 & 25 & .065 & .065 & .022 & 3 & 3 & 60 & 90 \\ 1006 & 25 & .065 & .065 & .022 & 3 & 3 & 15 & 90 \\ 1007 & 25 & .065 & .065 & .011 & 3 & 3 & 30 & 90 \\ 1008 & 25 & .065 & .065 & .0325 & 3 & 3 & 30 & 90 \\ 1009 & .06 & .065 & .065 & .0325 & 3 & 6 & 30 & 90 \\ 1010 & 25 & .065 & .065 & .0325 & 3 & -3 & 30 & 90 \\ \star 1011 & 25 & .065 & .065 & .0325 & 3 & 0 & 30 & 90\end{array}$

*Selected tip 
Two-element tip geometries

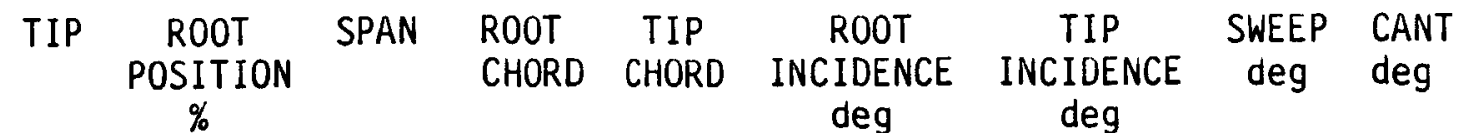

$\begin{array}{lllllllll}2000 & 12.5 & .02 & .0325 & .0325 & 3 & 3 & 0 & 90 \\ & 62.5 & .028 & .0325 & .0325 & 2 & 2 & 0 & 45 \\ 2001 & 12.5 & .04 & .0325 & .0325 & 3 & 3 & 0 & 90 \\ & 62.5 & .056 & .0325 & .0325 & 2 & 2 & 0 & 45 \\ 2002 & 12.5 & .028 & .0325 & .0325 & 2 & 2 & 0 & 45 \\ & 62.5 & .02 & .0325 & .0325 & 3 & 3 & 0 & 90 \\ 2003 & 12.5 & .056 & .0325 & .0325 & 2 & 2 & 0 & 45 \\ & 62.5 & .04 & .0325 & .0325 & 3 & 3 & 0 & 90 \\ 2004 & 12.5 & .056 & .0162 & .0162 & 2 & 2 & 0 & 45 \\ & 62.5 & .04 & .0162 & .0162 & 3 & 3 & 0 & 90 \\ 2005 & 12.5 & .056 & .0325 & .0325 & 0 & 0 & 0 & 45 \\ & 62.5 & .04 & .0325 & .0325 & 1 & 1 & 0 & 90 \\ 2006 & 12.5 & .056 & .0162 & .0162 & 0 & 0 & 0 & 45 \\ & 62.5 & .04 & .0162 & .0162 & 1 & 1 & 0 & 90 \\ 2007 & 12.5 & .056 & .0162 & .0162 & -2 & -2 & 0 & 45 \\ & 62.5 & .04 & .0162 & .0162 & -1 & -1 & 0 & 90 \\ 2008 & 12.5 & .056 & .008 & .008 & 2 & 2 & 0 & 45 \\ & 62.5 & .04 & .008 & .008 & 3 & 3 & 0 & 90 \\ 2009 & 12.5 & .056 & .0325 & .0325 & 2 & 2 & 0 & 45 \\ & 62.5 & .04 & .0162 & .0162 & 3 & 3 & 0 & 90 \\ 2010 & 12.5 & .056 & .0162 & .0162 & 2 & 2 & 0 & 45 \\ & 62.5 & .04 & .0325 & .0325 & 3 & 3 & 0 & 90 \\ 2011 & 12.5 & .056 & .0162 & .0162 & 0 & 0 & 0 & 45 \\ & 62.5 & .04 & .0325 & .0325 & 3 & 3 & 0 & 90 \\ 2012 & 12.5 & .056 & .0162 & .0162 & 2 & 2 & 0 & 45 \\ & 62.5 & .04 & .0325 & .0325 & 1 & 1 & 0 & 90 \\ 2013 & 12.5 & .056 & .0162 & .0162 & 0 & 0 & 0 & 45 \\ & 62.5 & .04 & .0325 & .0325 & 1 & 1 & 0 & 90 \\ 2014 & 31 & .056 & .0162 & .0162 & 2 & 2 & 0 & 45 \\ & 62.5 & .04 & .0325 & .0325 & 3 & 3 & 0 & 90 \\ 2015 & 12.5 & .056 & .0162 & .0162 & 2 & 2 & 0 & 45 \\ & 48 & .04 & .045 & .045 & 3 & 3 & 0 & 90\end{array}$


Two-element tip geometries

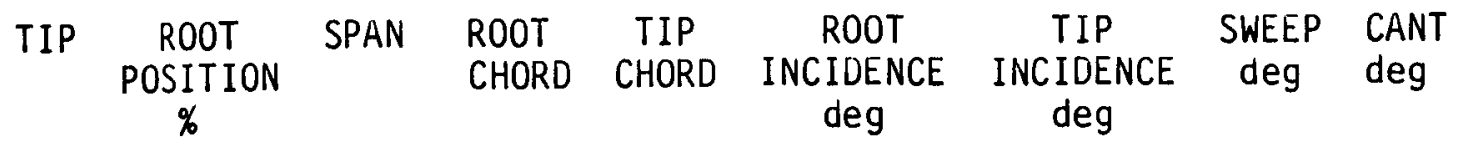

$\begin{array}{lllllllll}2016 & 31 & .056 & .0162 & .0162 & 0 & 0 & 0 & 45 \\ & 62.5 & .04 & .0325 & .0325 & 3 & 3 & 0 & 90 \\ 2017 & 31 & .056 & .0162 & .0162 & 2 & 2 & 0 & 45 \\ & 62.5 & .04 & .0325 & .0325 & 1 & 1 & 0 & 90 \\ 2018 & 31 & .056 & .0162 & .0162 & 0 & 0 & 0 & 45 \\ & 62.5 & .04 & .0325 & .0325 & 1 & 1 & 0 & 90 \\ 2019 & 24 & .056 & .0214 & .0107 & 2 & 2 & 8 & 45 \\ & 62.5 & .04 & .0325 & .0325 & 3 & 3 & 0 & 90 \\ 2020 & 31 & .056 & .0162 & .0162 & 2 & 2 & 0 & 45 \\ & 62.5 & .04 & .0325 & .0162 & 3 & 3 & 0 & 90 \\ 2021 & 31 & .056 & .0162 & .0162 & 2 & 2 & 0 & 45 \\ & 62.5 & .04 & .0325 & .0325 & 3 & 3 & 20 & 90 \\ 2022 & 31 & .056 & .0162 & .0162 & 2 & 2 & 0 & 45 \\ 2023 & .62 .5 & .04 & .0325 & .0162 & 3 & 3 & 20 & 90 \\ & 31 & .056 & .0162 & .0162 & 2 & 2 & 0 & 45 \\ 2024 & 62.5 & .04 & .0325 & .0081 & 3 & 3 & 0 & 90 \\ & 31 & .056 & .0162 & .0162 & 2 & 2 & 0 & 45 \\ 2025 & 62.5 & .04 & .0325 & .0243 & 3 & 3 & 0 & 90 \\ & 31 & .056 & .0162 & .0162 & 0 & 0 & 0 & 45 \\ 2026 & 31.5 & .04 & .0325 & .0162 & 1 & 1 & 0 & 90 \\ & 31.5 & .056 & .0162 & .0081 & 2 & 0 & 0 & 45 \\ & 62.5 & .04 & .0325 & .0162 & 3 & 1 & 0 & 90\end{array}$

*Selected tip 
Results for the single element tips

$$
\begin{array}{rrrrrrrrr}
\mathrm{V} & 70 & 35 & 23 & 17.4 & 13.9 & 11.6 & 9.9 & 8.7 \\
\mathrm{X} & 2 & 4 & 6 & 8 & 10 & 12 & 14 & 16
\end{array}
$$

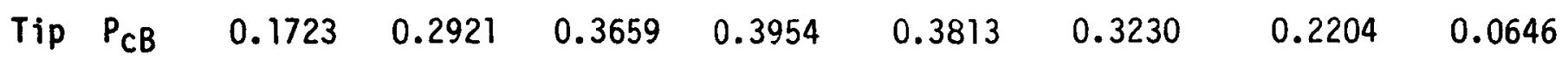

$\begin{array}{llllllllll}1 & P_{C} & 0.1770 & 0.2987 & 0.3727 & 0.4007 & 0.3839 & 0.3215 & 0.2128 & 0.0493\end{array}$

$\begin{array}{lllllllll}\text { \%P }_{C} & 0.0047 & 0.0066 & 0.0068 & 0.0053 & 0.0026 & -0.0015 & -0.0076 & -0.0153\end{array}$

$\begin{array}{llllllllll}2 & P_{C} & 0.1772 & 0.2986 & 0.3721 & 0.3990 & 0.3801 & 0.3143 & 0.2010 & 0.0314\end{array}$

$\begin{array}{lllllllll}\text { KP }_{c} & 0.0049 & 0.0065 & 0.0062 & 0.0036 & -0.0012 & -0.0089 & -0.0194 & -0.0332\end{array}$

$\begin{array}{llllllllll}3 & P_{C} & 0.1761 & 0.2967 & 0.3691 & 0.3945 & 0.3733 & 0.3041 & 0.1858 & 0.0098\end{array}$

$\begin{array}{lllllllll}\text { KP }_{C} & 0.0038 & 0.0046 & 0.0032 & -0.0009 & -0.0080 & -0.0189 & -0.0346 & -0.0548\end{array}$

$\begin{array}{lllllllll}4 & P_{C} & 0.1798 & 0.3021 & 0.3746 & 0.3989 & 0.3752 & 0.3023 & 0.1792\end{array}$

$\begin{array}{llllllll}\text { WP }_{C} & 0.0075 & 0.0100 & 0.0087 & 0.0035 & -0.0061 & -0.0207 & -0.0412\end{array}$

$\begin{array}{llllllllll}5 & P_{C} & 0.1772 & 0.2981 & 0.3712 & 0.3978 & 0.3798 & 0.3130 & 0.1995 & 0.0298\end{array}$

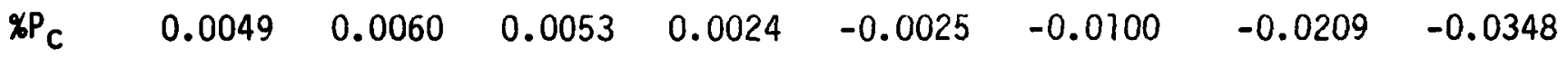

$\begin{array}{llllllllll}6 & P_{C} & 0.1740 & 0.2946 & 0.3681 & 0.3960 & 0.3789 & 0.3157 & 0.2059 & 0.0403\end{array}$

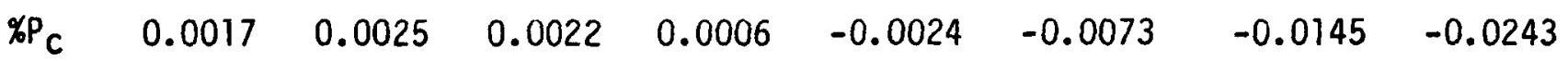

$\begin{array}{llllllllll}7 & P_{C} & 0.1758 & 0.2970 & 0.3710 & 0.3996 & 0.3838 & 0.3232 & 0.2170 & 0.0566\end{array}$

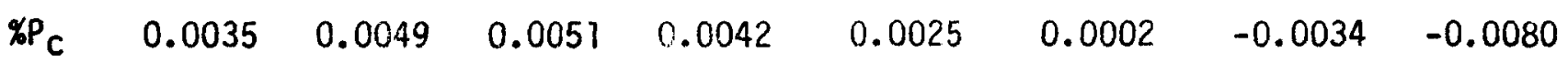

$\begin{array}{llllllllll}8 & P_{C} & 0.1743 & 0.2938 & 0.3671 & 0.3958 & 0.3807 & 0.3214 & 0.2173 & 0.0598\end{array}$

$\begin{array}{lllllllll}\text { KP }_{C} & 0.0020 & 0.0017 & 0.0012 & 0.0004 & -0.0006 & -0.0016 & -0.0031 & -0.0048\end{array}$

$\begin{array}{llllllllll}9 & P_{C} & 0.1717 & 0.2901 & 0.3634 & 0.3934 & 0.3815 & 0.3269 & 0.2296 & 0.0816\end{array}$ $\begin{array}{lllllllll}X P_{C} & -0.0006 & -0.0020 & -0.0025 & -0.0020 & 0.0002 & 0.0039 & 0.0092 & 0.0170\end{array}$

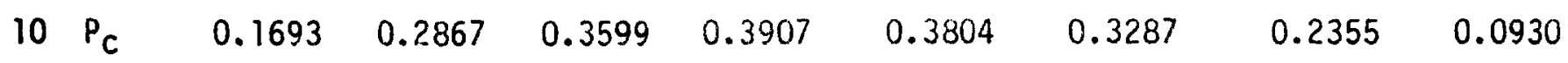
$\begin{array}{lllllllll}K P_{C} & -0.0030 & -0.0054 & -0.0060 & -0.0047 & -0.0009 & 0.0057 & 0.0151 & 0.0284\end{array}$ 
Results for the single element tips

$$
\begin{array}{rrrrrrrrr}
V & 70 & 35 & 23 & 17.4 & 13.9 & 11.6 & 9.9 & 8.7 \\
X & 2 & 4 & 6 & 8 & 10 & 12 & 14 & 16
\end{array}
$$

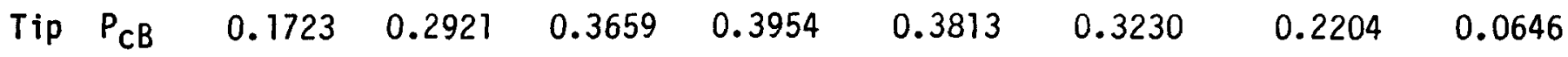

$\begin{array}{llllllllll}11 & P_{C} & 0.1675 & 0.2843 & 0.3572 & 0.3880 & 0.3780 & 0.3269 & 0.2346 & 0.0931\end{array}$

$\begin{array}{lllllllll}\% P_{C} & -0.0048 & -0.0078 & -0.0087 & -0.0074 & -0.0033 & 0.0039 & 0.0142 & 0.0285\end{array}$

$\begin{array}{llllllllll}12 & P_{C} & 0.1666 & 0.2834 & 0.3561 & 0.3864 & 0.3755 & 0.3229 & 0.2288 & 0.0846\end{array}$ $\begin{array}{lllllllll}\text { \%P } & -0.0057 & -0.0087 & -0.0098 & -0.0090 & -0.0058 & -0.0001 & 0.0084 & 0.0200\end{array}$

$\begin{array}{llllllllll}13 & P_{C} & 0.1674 & 0.2849 & 0.3576 & 0.3870 & 0.3740 & 0.3182 & 0.2195 & 0.0690\end{array}$ $\begin{array}{lllllllll}\% P_{C} & -0.0049 & -0.0072 & -0.0083 & -0.0084 & -0.0073 & -0.0048 & -0.0009 & 0.0044\end{array}$

$\begin{array}{llllllllll}14 & P_{C} & 0.1700 & 0.2890 & 0.3620 & 0.3902 & 0.3743 & 0.3138 & 0.2080 & 0.0480\end{array}$ $\begin{array}{lllllllll}\% P_{C} & -0.0023 & -0.0031 & -0.0039 & -0.0052 & -0.0070 & -0.0092 & -0.0124 & -0.0166\end{array}$

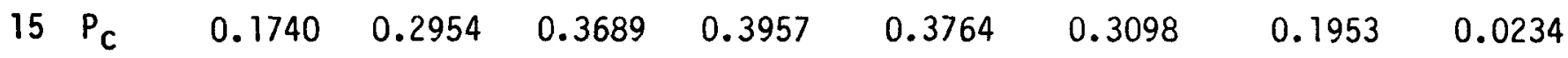
$\begin{array}{lllllllll}\% P_{C} & 0.0017 & 0.0033 & 0.0030 & 0.0003 & -0.0049 & -0.0132 & -0.0251 & -0.0412\end{array}$

$\begin{array}{llllllllll}16 & P_{C} & 0.1771 & 0.2991 & 0.3737 & 0.4026 & 0.3868 & 0.3257 & 0.2188 & 0.0578\end{array}$

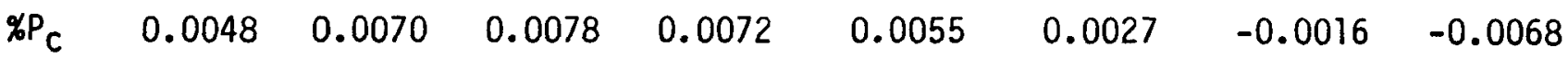

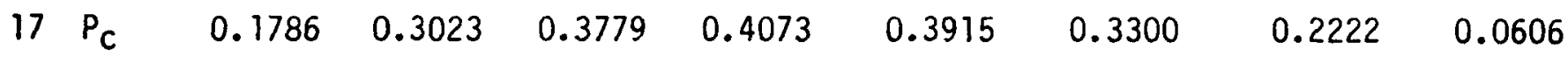
$\begin{array}{lllllllll}\text { XP }_{C} & 0.0063 & 0.0102 & 0.0120 & 0.0119 & 0.0102 & 0.0070 & 0.0018 & -0.0046\end{array}$

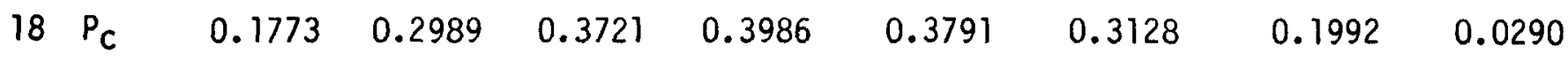

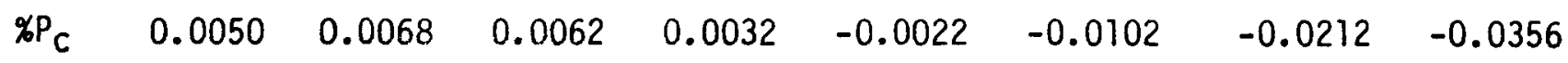

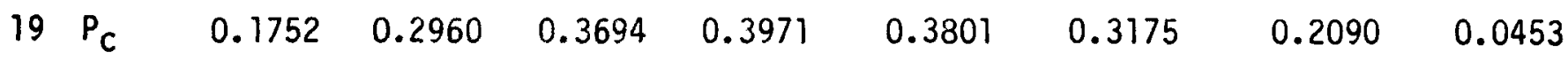
$\begin{array}{lllllllll}\text { \%P } & 0.0029 & 0.0039 & 0.0035 & 0.0017 & -0.0012 & -0.0055 & -0.0114 & -0.0193\end{array}$

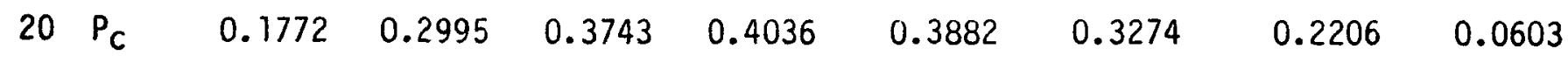
$\begin{array}{lllllllll}\% P_{C} & 0.0049 & 0.0074 & 0.0084 & 0.0082 & 0.0069 & 0.0044 & 0.0002 & -0.0043\end{array}$ 
Results for the single element tips

$$
\begin{array}{rrrrrrrrr}
V & 70 & 35 & 23 & 17.4 & 13.9 & 11.6 & 9.9 & 8.7 \\
X & 2 & 4 & 6 & 8 & 10 & 12 & 14 & 16
\end{array}
$$

$\begin{array}{llllllllll}\text { Tip } & P_{C B} & 0.1723 & 0.2921 & 0.3659 & 0.3954 & 0.3813 & 0.3230 & 0.2204 & 0.0646\end{array}$ $\begin{array}{llllllllll}21 & P_{C} & 0.1773 & 0.2998 & 0.3748 & 0.4039 & 0.3881 & 0.3265 & 0.2194 & 0.0568\end{array}$ $\begin{array}{lllllllll}\% P_{C} & 0.0050 & 0.0077 & 0.0089 & 0.0085 & 0.0068 & 0.0035 & -0.0020 & -0.0078\end{array}$ $\begin{array}{llllllllll}22 & \mathrm{P}_{\mathrm{C}} & 0.1785 & 0.3018 & 0.3766 & 0.4050 & 0.3883 & 0.3259 & 0.2174 & 0.0542\end{array}$ $\begin{array}{lllllllll}\text { KP }_{C} & 0.0062 & 0.0097 & 0.0107 & 0.0096 & 0.0070 & 0.0029 & -0.0030 & -0.0104\end{array}$

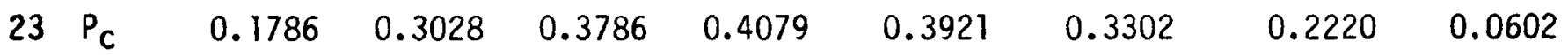
$\begin{array}{lllllllll}x_{C} & 0.0063 & 0.0107 & 0.0127 & 0.0125 & 0.0108 & 0.0072 & 0.0016 & -0.0044\end{array}$ $\begin{array}{llllllllll}24 & P_{C} & 0.1785 & 0.3024 & 0.3781 & 0.4072 & 0.3909 & 0.3282 & 0.2185 & 0.0550\end{array}$ $\begin{array}{lllllllll}\text { KP } & 0.0062 & 0.0103 & 0.0122 & 0.0118 & 0.0096 & 0.0052 & -0.0019 & -0.0096\end{array}$ $\begin{array}{llllllllll}25 & P_{C} & 0.1816 & 0.3053 & 0.3801 & 0.4082 & 0.3910 & 0.3278 & 0.2187 & 0.0547\end{array}$ $\begin{array}{lllllllll}\text { KP } & 0.0093 & 0.0132 & 0.0142 & 0.0128 & 0.0097 & 0.0048 & -0.0017 & -0.0099\end{array}$

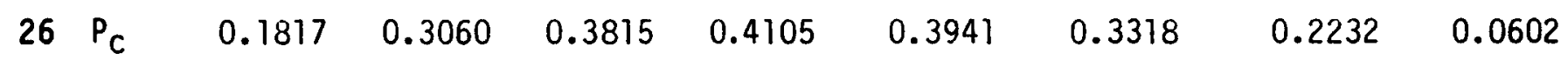
$\begin{array}{lllllllll}\text { \%P } & 0.0094 & 0.0139 & 0.0156 & 0.0151 & 0.0128 & 0.0088 & 0.0028 & -0.0044\end{array}$ $\begin{array}{llllllllll}27 & P_{C} & 0.1819 & 0.3066 & 0.3827 & 0.4120 & 0.3958 & 0.3334 & 0.2240 & 0.0600\end{array}$ $\begin{array}{lllllllll}\text { \%P } & 0.0096 & 0.0145 & 0.0168 & 0.0166 & 0.0145 & 0.0104 & 0.0036 & -0.0046\end{array}$

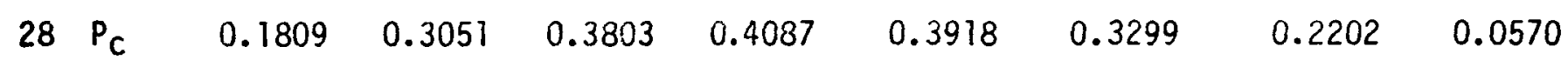
$\begin{array}{lllllllll}\text { \%P } & 0.0086 & 0.0130 & 0.0144 & 0.0133 & 0.0105 & 0.0069 & -0.0002 & -0.0076\end{array}$ $\begin{array}{llllllllll}29 & P_{C} & 0.1819 & 0.3053 & 0.3800 & 0.4082 & 0.3910 & 0.3281 & 0.2188 & 0.0550\end{array}$

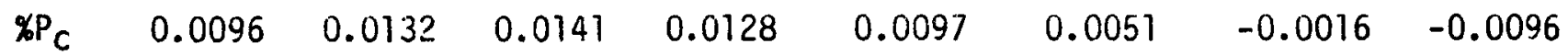
$\begin{array}{llllllllll}30 & P_{C} & 0.1820 & 0.3059 & 0.3811 & 0.4097 & 0.3927 & 0.3298 & 0.2199 & 0.0555\end{array}$ $\begin{array}{lllllllll}\% P_{c} & 0.0097 & 0.0138 & 0.0152 & 0.0143 & 0.0114 & 0.0068 & -0.0005 & -0.0096\end{array}$ 
Results for the single element tips

$$
\begin{array}{rrrrrrrrr}
V & 70 & 35 & 23 & 17.4 & 13.9 & 11.6 & 9.9 & 8.7 \\
X & 2 & 4 & 6 & 8 & 10 & 12 & 14 & 16
\end{array}
$$

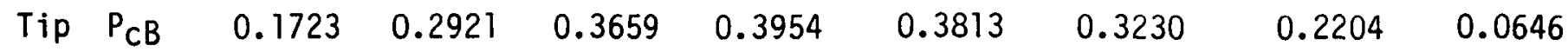

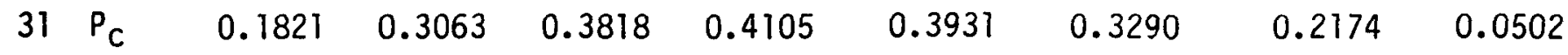
$\begin{array}{lllllllll}\% P_{C} & 0.0098 & 0.0142 & 0.0159 & 0.0151 & 0.0118 & 0.0060 & -0.0030 & -0.0144\end{array}$

$\begin{array}{llllllllll}32 & P_{C} & 0.1813 & 0.3047 & 0.3794 & 0.4077 & 0.3906 & 0.3278 & 0.2190 & 0.0555\end{array}$ $\begin{array}{lllllllll}\% P_{C} & 0.0090 & 0.0126 & 0.0135 & 0.0123 & 0.0093 & 0.0048 & -0.0014 & -0.0091\end{array}$

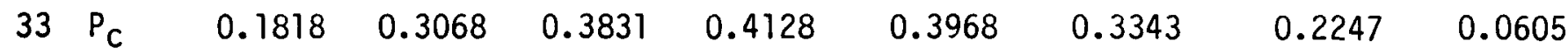
$\begin{array}{lllllllll}\text { \%P }_{C} & 0.0095 & 0.0147 & 0.0172 & 0.0174 & 0.0155 & 0.0118 & 0.0043 & -0.0041\end{array}$

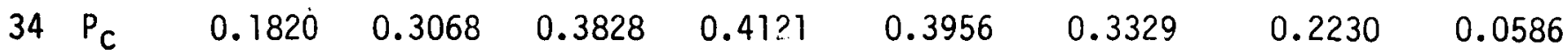
$\begin{array}{lllllllll}\text { \%P } & 0.0097 & 0.0147 & 0.0169 & 0.0167 & 0.0143 & 0.0099 & 0.0026 & -0.0060\end{array}$

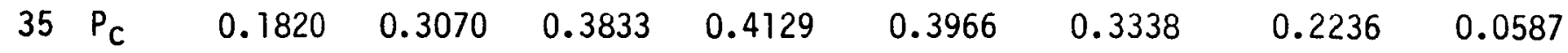
$\begin{array}{lllllllll}\%_{\mathrm{C}} \mathrm{P}_{\mathrm{C}} & 0.0097 & 0.0149 & 0.0174 & 0.0175 & 0.0153 & 0.0108 & 0.0032 & -0.0059\end{array}$

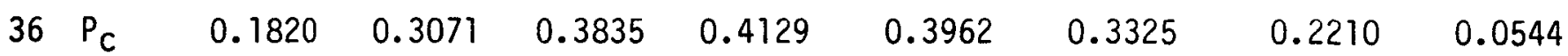
$\begin{array}{lllllllll}\% \mathrm{P}_{\mathrm{C}} & 0.0097 & 0.0150 & 0.0176 & 0.0175 & 0.0149 & 0.0095 & 0.0006 & -0.0102\end{array}$

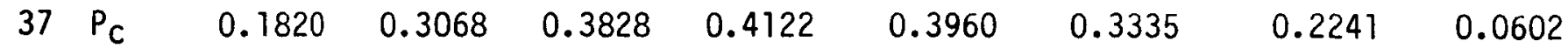
$\begin{array}{lllllllll}\% P_{C} & 0.0097 & 0.0147 & 0.0169 & 0.0168 & 0.0147 & 0.0105 & 0.0037 & -0.0044\end{array}$

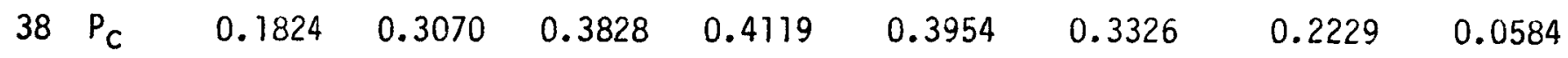
$\begin{array}{lllllllll}\% P_{C} & 0.0101 & 0.0149 & 0.0169 & 0.0165 & 0.0141 & 0.0096 & 0.0025 & -0.0062\end{array}$ $\begin{array}{llllllllll}\star 39 & P_{C} & 0.1825 & 0.3072 & 0.3831 & 0.4122 & 0.3959 & 0.3332 & 0.2237 & 0.0595\end{array}$ $\begin{array}{lllllllll}\% P_{C} & 0.0102 & 0.0151 & 0.0172 & 0.0168 & 0.0146 & 0.0103 & 0.0033 & -0.0051\end{array}$

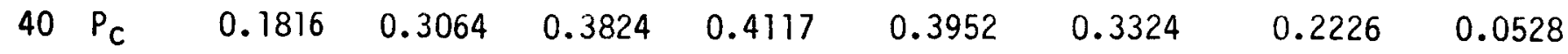
$\begin{array}{lllllllll}\% P_{C} & 0.0093 & 0.0143 & 0.0165 & 0.0163 & 0.0139 & 0.0094 & 0.0022 & -0.0118\end{array}$ 
Results for the single element tips

$$
\begin{array}{rrrrrrrrr}
\mathrm{V} & 70 & 35 & 23 & 17.4 & 13.9 & 11.6 & 9.9 & 8.7 \\
\mathrm{X} & 2 & 4 & 6 & 8 & 10 & 12 & 14 & 16
\end{array}
$$

\begin{tabular}{|c|c|c|c|c|c|c|c|c|c|}
\hline Tip & $P_{C B}$ & 0.1723 & 0.2921 & 0.3659 & 0.3954 & 0.3813 & 0.3230 & 0.2204 & 0.0646 \\
\hline \multirow[t]{2}{*}{41} & $\mathrm{P}_{\mathrm{C}}$ & 0.1819 & 0.3064 & 0.3824 & 0.4118 & 0.3959 & 0.3337 & 0.2250 & 0.0616 \\
\hline & $\% P_{C}$ & 0.0096 & 0.0143 & 0.0165 & 0.0164 & 0.0146 & 0.0107 & 0.0046 & -0.0030 \\
\hline \multirow[t]{2}{*}{42} & $\mathrm{P}_{\mathrm{C}}$ & 0.1811 & 0.3055 & 0.3815 & 0.4114 & 0.3962 & 0.3352 & 0.2279 & 0.0667 \\
\hline & $\mathscr{H} P_{c}$ & 0.0088 & 0.0134 & 0.0156 & 0.0160 & 0.0149 & 0.0122 & 0.0075 & 0.0021 \\
\hline \multirow[t]{2}{*}{43} & $\mathrm{P}_{\mathrm{c}}$ & 0.1814 & 0.3057 & 0.3814 & 0.4105 & 0.3940 & 0.3313 & 0.2218 & 0.0576 \\
\hline & ${ }^{*} P_{c}$ & 0.0091 & 0.0136 & 0.0155 & 0.0151 & 0.0127 & 0.0083 & 0.0014 & -0.0070 \\
\hline \multirow[t]{2}{*}{44} & $P_{c}$ & 0.1777 & 0.2991 & 0.3724 & 0.3994 & 0.3810 & 0.3164 & 0.2050 & 0.0386 \\
\hline & ${ }^{*} P_{C}$ & 0.0054 & 0.0070 & 0.0065 & 0.0040 & -0.0003 & -0.0066 & -0.0154 & -0.0260 \\
\hline \multirow[t]{2}{*}{45} & $P_{c}$ & 0.1768 & 0.2988 & 0.3721 & 0.3985 & 0.3791 & 0.3132 & 0.2005 & 0.0320 \\
\hline & $\% P_{c}$ & 0.0045 & 0.0067 & 0.0062 & 0.0031 & -0.0022 & -0.0098 & -0.0199 & -0.0326 \\
\hline
\end{tabular}


Results for "Shark fin" type tip

$$
\begin{array}{rrrrrrrrr}
V & 70 & 35 & 23 & 17.4 & 13.9 & 11.6 & 9.9 & 8.7 \\
X & 2 & 4 & 6 & 8 & 10 & 12 & 14 & 16
\end{array}
$$

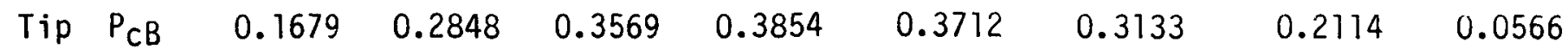

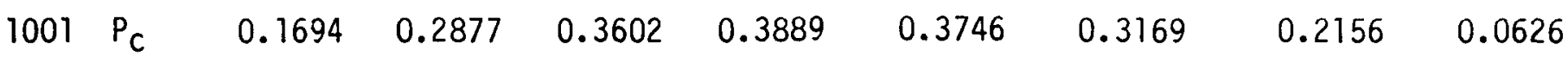

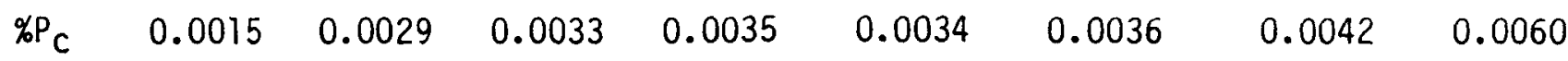

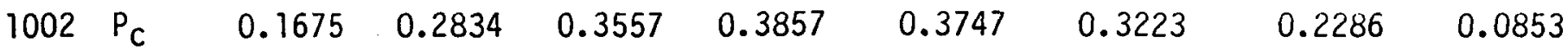

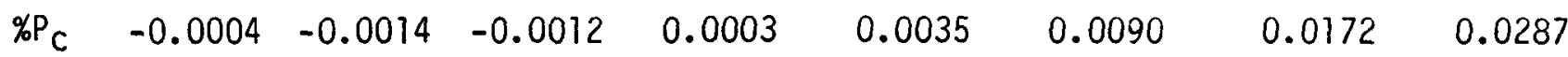

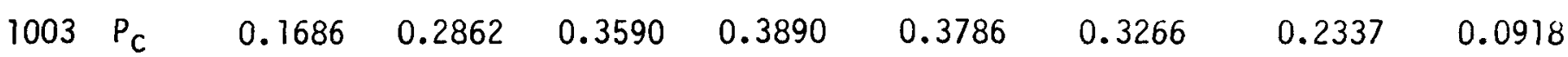
$\begin{array}{lllllllll}\text { \% }_{C} & 0.0007 & 0.0014 & 0.0021 & 0.0036 & 0.0074 & 0.0133 & 0.0223 & 0.0352\end{array}$

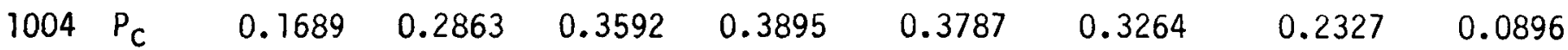
$\begin{array}{lllllllll}\% P_{C} & 0.0010 & 0.0015 & 0.0023 & 0.0041 & 0.0075 & 0.0131 & 0.0213 & 0.0330\end{array}$

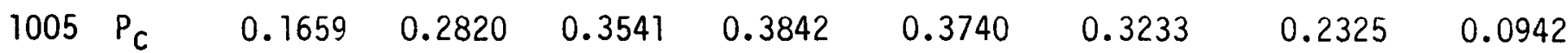
$\begin{array}{lllllllll}\text { \%P }_{C} & -0.0020 & -0.0028 & -0.0028 & -0.0012 & 0.0028 & 0.0100 & 0.0211 & 0.0376\end{array}$

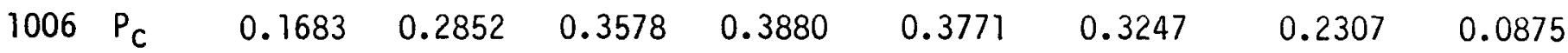
$\begin{array}{lllllllll}\text { \% }_{C} & 0.0004 & 0.0004 & 0.0009 & 0.0026 & 0.0059 & 0.0114 & 0.0193 & 0.0309\end{array}$

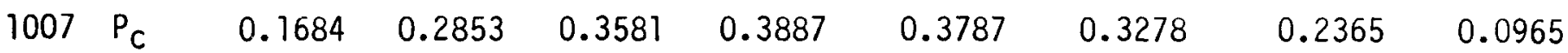
$\begin{array}{lllllllll}\%_{C} P_{C} & 0.0005 & 0.0005 & 0.0012 & 0.0033 & 0.0075 & 0.0145 & 0.0251 & 0.0399\end{array}$ $\begin{array}{llllllllll}1008 & P_{C} & 0.1691 & 0.2868 & 0.3598 & 0.3898 & 0.3781 & 0.3244 & 0.2286 & 0.0827\end{array}$ $\begin{array}{lllllllll}\% P_{C} & 0.0012 & 0.0020 & 0.0029 & 0.0044 & 0.0069 & 0.0111 & 0.0172 & 0.0261\end{array}$

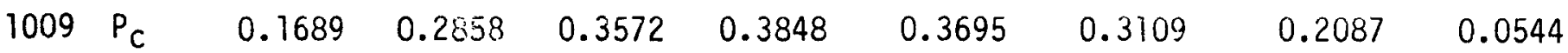
$\begin{array}{lllllllll}\%_{C} & 0.0010 & 0.0010 & 0.0003 & -0.0006 & -0.0017 & -0.0024 & -0.0027 & -0.0022\end{array}$

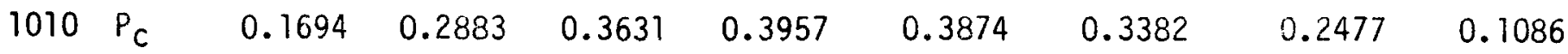
$\begin{array}{lllllllll}\text { \%P } & 0.0015 & 0.0035 & 0.0062 & 0.0103 & 0.0162 & 0.0249 & 0.0363 & 0.0520\end{array}$

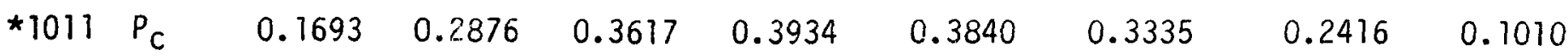

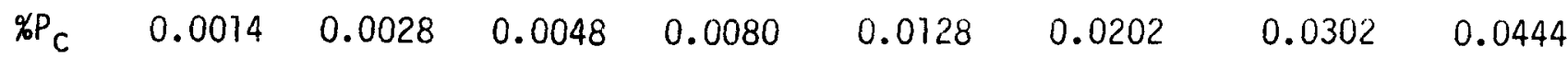


Results for two element tips

$$
\begin{array}{rrrrrrrrr}
V & 70 & 35 & 23 & 17.4 & 13.9 & 11.6 & 9.9 & 8.7 \\
X & 2 & 4 & 6 & 8 & 10 & 12 & 14 & 16
\end{array}
$$

$\begin{array}{lllllllll}\text { Tip } P_{C B} & 0.1723 & 0.2921 & 0.3659 & 0.3954 & 0.3813 & 0.3230 & 0.2204 & 0.0646\end{array}$ $\begin{array}{llllllllll}2000 & P_{C} & 0.1710 & 0.2804 & 0.3627 & 0.3968 & 0.3833 & 0.3282 & 0.2254 & 0.0680\end{array}$ $\begin{array}{lllllllll}\% P_{C} & -0.0013 & -0.0117 & -0.0032 & 0.0014 & 0.0020 & 0.0052 & 0.0050 & 0.0034\end{array}$

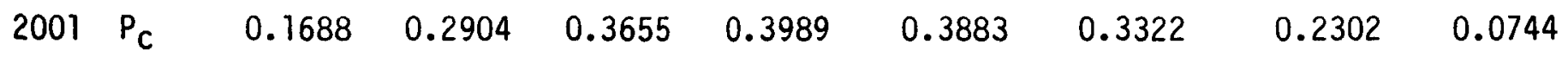

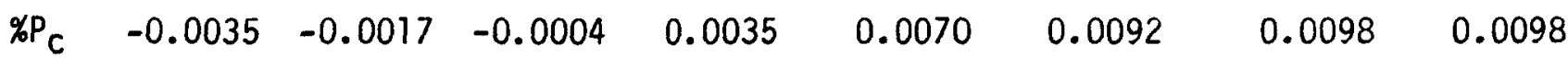
$\begin{array}{llllllllll}2002 & P_{C} & 0.1701 & 0.2870 & 0.3617 & 0.3932 & 0.3811 & 0.3241 & 0.2213 & 0.0640\end{array}$

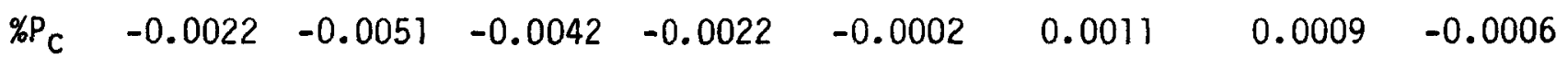

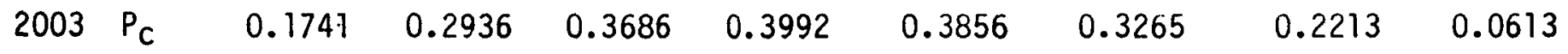
$\begin{array}{lllllllll}\text { \% } P_{C} & 0.0018 & 0.0039 & 0.0027 & 0.0038 & 0.0043 & 0.0035 & 0.0003 & -0.0033\end{array}$ $\begin{array}{llllllllll}2004 & P_{C} & 0.1767 & 0.2968 & 0.3712 & 0.4025 & 0.3910 & 0.3365 & 0.2388 & 0.0898\end{array}$ $\begin{array}{lllllllll}\% P_{C} & 0.0044 & 0.0047 & 0.0053 & 0.0071 & 0.0097 & 0.0135 & 0.0184 & 0.0252\end{array}$

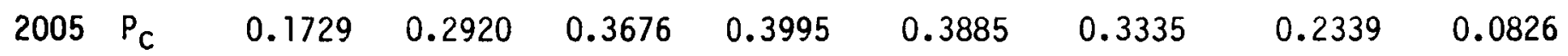

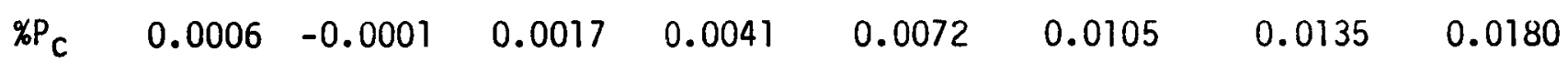
$\begin{array}{llllllllll}2006 & P_{C} & 0.1770 & 0.2973 & 0.3734 & 0.4071 & 0.3993 & 0.3494 & 0.2579 & 0.1171\end{array}$ $\begin{array}{lllllllll}\% P_{C} & 0.0047 & 0.0052 & 0.0075 & 0.0117 & 0.0180 & 0.0264 & 0.0375 & 0.0524\end{array}$ $\begin{array}{llllllllll}2007 & P_{C} & 0.1772 & 0.2978 & 0.3749 & 0.4102 & 0.4044 & 0.3574 & 0.2691 & 0.1328\end{array}$ $\begin{array}{lllllllll}\%_{C} & 0.0049 & 0.0057 & 0.0090 & 0.0148 & 0.0231 & 0.0344 & 0.0487 & 0.0682\end{array}$

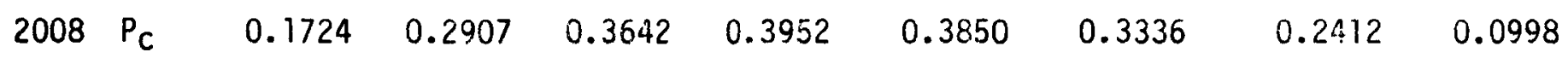
$\begin{array}{lllllllll}\text { KP } & 0.0001 & -0.0014 & -0.0017 & -0.0002 & 0.0037 & 0.0106 & 0.0208 & 0.0352\end{array}$ $\begin{array}{llllllllll}2009 & P_{C} & 0.1732 & 0.2922 & 0.3670 & 0.3982 & 0.3861 & 0.3298 & 0.2286 & 0.0742\end{array}$ $\begin{array}{lllllllll}\text { \%P } & 0.0009 & -0.0001 & 0.0011 & 0.0028 & 0.0048 & 0.0068 & 0.0082 & 0.0096\end{array}$ $\begin{array}{llllllllll}2010 & P_{C} & 0.1785 & 0.2989 & 0.3737 & 0.4042 & 0.3911 & 0.3336 & 0.2317 & 0.0768\end{array}$ $\begin{array}{lllllllll}\%_{C} & 0.0062 & 0.0068 & 0.0078 & 0.0088 & 0.0098 & 0.0106 & 0.0113 & 0.0122\end{array}$ 
Results for two element tips

$$
\begin{array}{lrrrrrrrr}
\mathrm{V} & 70 & 35 & 23 & 17.4 & 13.9 & 11.6 & 9.9 & 8.7 \\
\mathrm{X} & 2 & 4 & 6 & 8 & 10 & 12 & 14 & 16
\end{array}
$$

\begin{tabular}{|c|c|c|c|c|c|c|c|c|c|}
\hline Tip & $P_{C B}$ & 0.1723 & 0.2921 & 0.3659 & 0.3954 & 0.3813 & 0.3230 & 0.2204 & 0.0646 \\
\hline \multirow[t]{2}{*}{2011} & $P_{C}$ & 0.1785 & 0.2988 & 0.3742 & 0.4059 & 0.3948 & 0.3403 & 0.2425 & - \\
\hline & $\% P_{c}$ & 0.0062 & 0.0067 & 0.0083 & 0.0105 & 0.0135 & 0.0173 & 0.0221 & - \\
\hline \multirow[t]{2}{*}{2012} & $P_{c}$ & 0.1786 & 0.2995 & 0.3751 & 0.4068 & 0.3953 & 0.3398 & 0.2404 & - \\
\hline & $P_{c}$ & 0.0003 & 0.0074 & 0.0092 & 0.0114 & 0.0140 & 0.0168 & 0.0200 & - \\
\hline \multirow[t]{2}{*}{2013} & $P_{C}$ & 0.1786 & 0.2994 & 0.3755 & 0.4084 & 0.3988 & 0.3462 & 0.2506 & - \\
\hline & ${ } P_{C}$ & 0.0063 & 0.0073 & 0.0096 & 0.0130 & 0.0175 & 0.0232 & 0.0302 & - \\
\hline \multirow[t]{2}{*}{2014} & $P_{C}$ & 0.1797 & 0.3014 & 0.3770 & 0.4081 & 0.3953 & 0.3383 & 0.2372 & - \\
\hline & ${ }^{2} P_{C}$ & 0.0074 & 0.0093 & 0.0111 & 0.0127 & 0.0140 & 0.0153 & 0.0168 & - \\
\hline \multirow[t]{2}{*}{2015} & $P_{c}$ & 0.1768 & 0.2970 & 0.3718 & 0.4018 & 0.3877 & 0.3284 & 0.2240 & - \\
\hline & ${ }^{2} \mathrm{P}_{\mathrm{C}}$ & 0.0045 & 0.0049 & 0.0059 & 0.0064 & 0.0064 & 0.0054 & 0.0036 & - \\
\hline \multirow[t]{2}{*}{2016} & $P_{c}$ & 0.1794 & 0.3010 & 0.3775 & 0.4096 & 0.3986 & 0.3439 & 0.2458 & 0.0965 \\
\hline & $\mathscr{H} P_{c}$ & 0.0071 & 0.0089 & 0.0116 & 0.0142 & 0.0173 & 0.0209 & 0.0254 & 0.0319 \\
\hline \multirow[t]{2}{*}{2017} & $P_{c}$ & 0.1798 & 0.3019 & 0.3783 & 0.4103 & 0.3989 & 0.3438 & 0.2450 & 0.0941 \\
\hline & ${ }^{*} \mathrm{P}_{\mathrm{C}}$ & 0.0075 & 0.0098 & 0.0124 & 0.0149 & 0.0176 & 0.0208 & 0.0246 & 0.0295 \\
\hline \multirow[t]{2}{*}{2018} & $P_{c}$ & 0.1795 & 0.3015 & 0.3786 & 0.4117 & 0.4020 & 0.3491 & 0.2531 & 0.1066 \\
\hline & $\% P_{c}$ & 0.0072 & 0.0094 & 0.0127 & 0.0163 & 0.0207 & 0.0261 & 0.0327 & 0.0420 \\
\hline \multirow[t]{2}{*}{2019} & $P_{C}$ & 0.1783 & 0.2991 & 0.3747 & 0.4060 & 0.3937 & 0.3372 & 0.2366 & 0.0832 \\
\hline & $\% P_{C}$ & 0.0060 & 0.0070 & 0.0088 & 0.0106 & 0.0124 & 0.0142 & 0.0162 & 0.0186 \\
\hline \multirow[t]{2}{*}{ *2020 } & $P_{C}$ & 0.1796 & 0.3010 & 0.3770 & 0.4088 & 0.3974 & 0.3427 & 0.2449 & 0.0954 \\
\hline & ${ } P_{C}$ & 0.0073 & 0.0089 & 0.0111 & 0.0134 & 0.0161 & 0.0197 & 0.0245 & 0.0308 \\
\hline
\end{tabular}

* Selected

Design 


\section{Results for two element tips}

$$
\begin{array}{rrrrrrrrr}
V & 70 & 35 & 23 & 17.4 & 13.9 & 11.6 & 9.9 & 8.7 \\
X & 2 & 4 & 6 & 8 & 10 & 12 & 14 & 16
\end{array}
$$

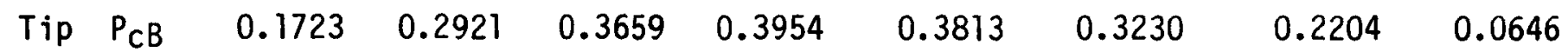

$$
2021 P_{C}
$$

$\begin{array}{lll}P_{C} & 0.1802 & 0.3018\end{array}$

$0.3777 \quad 0.4089 \quad 0.3963$

0.3395

0.2386

0.0850

$\% \mathrm{P}_{\mathrm{C}}$

0.0079

0.0097

0.01180 .0135

0.0150

0.0165

0.0185

0.0204

$2022 P_{C}$

$0.1800 \quad 0.3013$

$0.3770 \quad 0.4086$

0.3967

0.3412

0.2422

0.0914 $\% \mathrm{P}_{\mathrm{C}}$

$\begin{array}{ll}0.0075 & 0.0092\end{array}$

0.01180 .0132

0.0154

0.0182

0.0218

0.0268

$2023 P_{C}$

$\begin{array}{ll}0.1792 & 0.3001\end{array}$

$0.3755 \quad 0.4068$

0.3950

0.3400

0.2418

0.0923

$\% P_{c}$

$0.0069 \quad 0.0080$

$0.0096 \quad 0.0114$

0.0137

0.0170

0.0214

0.0277

$2024 P_{C}$

$\begin{array}{lll}0.1796 & 0.3011\end{array}$

$0.3768 \quad 0.4079$

0.3954

0.3391

0.2388

0.0864

$\% P_{C}$

$0.0073 \quad 0.0090$

$0.0109 \quad 0.0125$

0.0141

0.0161

0.0184

0.0218

$2025 P_{C}$

$0.1793 \quad 0.3010$

$0.3780 \quad 0.4114$

0.4023

0.3506

0.2565

0.1123

$\% P_{C}$

0.0070

0.0089

0.01210 .0160

0.0210

0.0276

0.0361

0.0477

$2026 P_{C}$

$\begin{array}{llll}0.1791 & 0.3004 & 0.3766 & 0.4090\end{array}$

0.3989

0.3461

0.2506

0.1046

$\% P_{C}$

0.0068

0.0083

$0.0107 \quad 0.0136$

0.0176

0.0231

0.0302

0.0400 


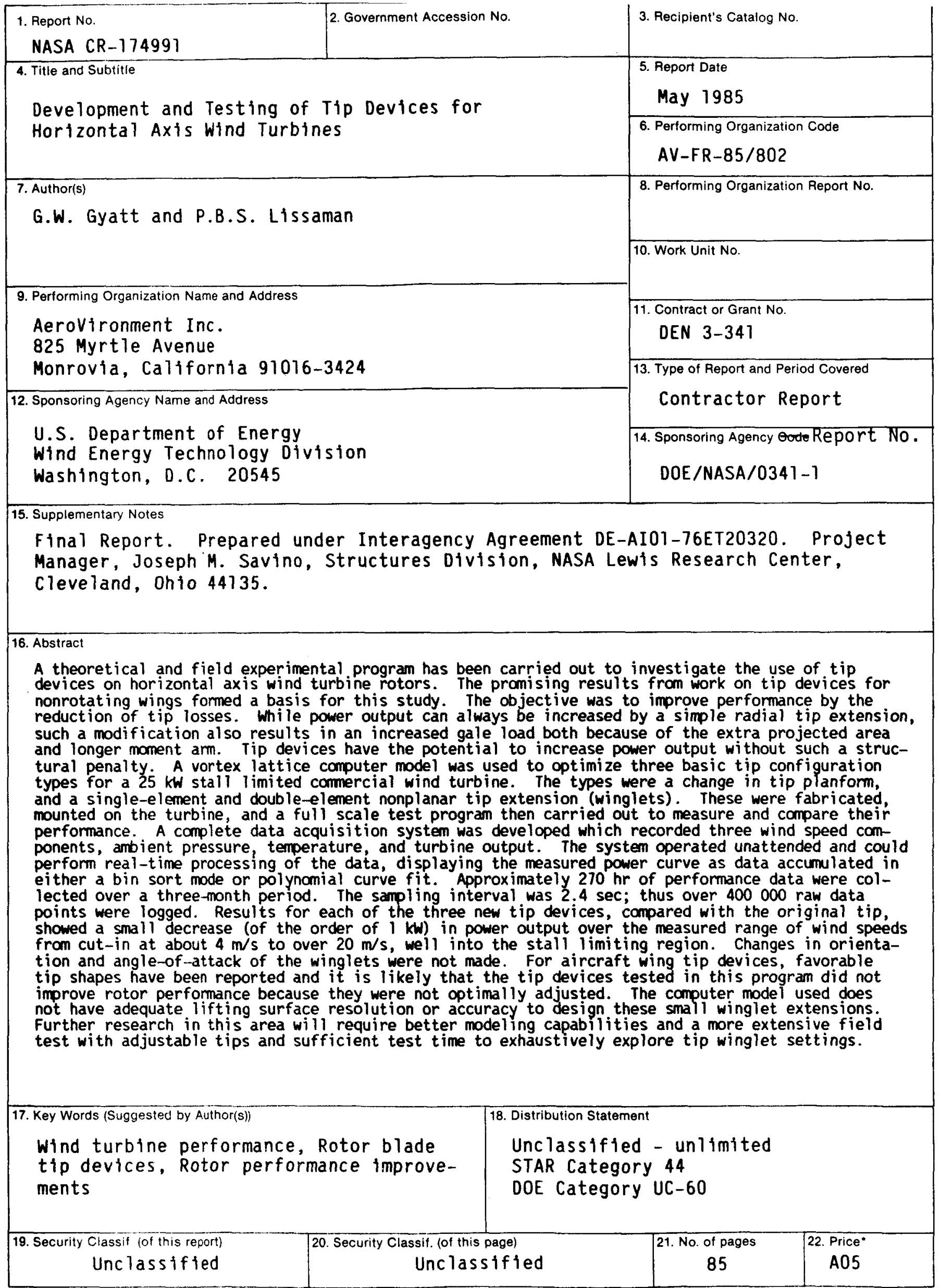

"For sale by the National Technical Information Service, Soringfield, Virginia 22161 